Claremont Colleges

Scholarship@Claremont

KGI Theses and Dissertations

KGI Student Scholarship

5-18-2013

\title{
Device Engineering for Infectious Disease Diagnosis using Isothermal DNA Amplification and Lateral Flow Detection
}

Kristina Roskos

Keck Graduate Institute

\section{Recommended Citation}

Roskos, Kristina, "Device Engineering for Infectious Disease Diagnosis using Isothermal DNA Amplification and Lateral Flow Detection" (2013). KGI Theses and Dissertations. Paper 2.

http://scholarship.claremont.edu/kgi_theses/2

DOI: $10.5642 / \mathrm{kgitd} / 2$

This Open Access Dissertation is brought to you for free and open access by the KGI Student Scholarship at Scholarship @ Claremont. It has been accepted for inclusion in KGI Theses and Dissertations by an authorized administrator of Scholarship @ Claremont. For more information, please contact scholarship@cuc.claremont.edu. 
Device Engineering for Infectious Disease Diagnosis using Isothermal DNA Amplification and Lateral Flow Detection

\section{BY}

Kristina Roskos 
We, the undersigned, certify that we have read this dissertation of Kristina Roskos and approve it as adequate in scope and quality for the degree of Doctor of Philosophy.

Dissertation Committee:

Dr. Angelika Niemz

Professor, Chair

Dr. Jim Sterling

Member

$\underline{\text { Dr. Jim Osborne }}$

Member

Dr. Robert Matson

Member

Dr. Animesh Ray

PhD Program Director 
Device Engineering for Infectious Disease Diagnosis using Isothermal DNA Amplification and Lateral Flow Detection

\section{BY}

\section{Kristina Roskos}

A Dissertation submitted to the Faculty of Keck Graduate Institute of Applied Life Sciences in partial fulfillment of the requirements for the degree of Doctor of Philosophy in Applied Life Sciences

\section{Claremont, California}

2013

Approved by:

Angelika Niemz Ph.D.

Arnold and Mabel Beckman Professor and Director of Research Keck Graduate Institute, Doctoral Thesis Advisor 


\title{
Abstract of the Dissertation
}

\section{Device Engineering for Infectious Disease Diagnosis using Isothermal DNA Amplification and Lateral Flow Detection}

\author{
By \\ Kristina Roskos \\ Keck Graduate Institute of Applied Life Sciences: 2013
}

Technologies that enable infectious diseases diagnosis in low-resource settings could greatly facilitate effective treatment and containment of such diseases. Nucleic acid amplification testing can be used to identify pathogens, but typically requires highlytrained personnel and large, expensive lab equipment, neither of which is available in low-resource settings. Our overall goal is to develop a portable diagnostic system that utilizes a low-cost, disposable, mesofluidic cartridge and a handheld electronics unit to perform fully-integrated nucleic acid testing at the point of care in low-resource settings. As a first step toward this goal, we developed a subunit to execute isothermal nucleic acid amplification coupled with lateral flow detection, in parallel with the development of a sample preparation subunit by our collaborators at Claremont BioSolutions. Fluid handling inside the amplification and detection cartridge is facilitated through one-way passive valves, flexible pouches, and electrolysis-driven pumps, which promotes a compact and inexpensive instrument design. The closed-system disposable prevents 
workspace amplicon contamination. The cartridge design is based on standard, scalable manufacturing techniques, such as injection molding. Using an initial prototype system, we demonstrated detection of purified Mycobacterium tuberculosis genomic DNA. We then developed a refined amplification and detection cartridge in conjunction with an improved portable instrument, which automates pumping, heating, and timing, using a design format compatible with eventual integration with the sample preparation subunit. This refined cartridge incorporates a novel, inexpensive, stand-alone, passive valve, smaller, integrated pump components, a more complex injection molded polycarbonate cartridge core piece, and enhanced lateral flow chambers to improve visual detection. The independent valve component can be tailored for a variety of fluidic systems. We demonstrated appropriate fluidic and thermal control, and successful isothermal nucleic acid amplification within this refined amplification and detection subunit. We have developed a separate fluidic module for master-mix reagent storage and reconstitution that is designed to act as the interface between the amplification and detection subunit and the upstream sample preparation subunit. We envision that the merger of these two subunits into a fully-integrated cartridge will enable user-friendly, automated sample-in to answer-out diagnosis of infectious diseases in primary care settings of low-resource countries with high disease burden. 


\section{Dedication}

I thank my parents, Patricia and Henry Roskos, for their unwavering support in all of my endeavors. 


\section{ACKNOWLEDGMENTS}

First and foremost, I thank the engineers and scientists who I work with regularly: Anna Hickerson, Hsiang-Wei Lu, Tanya Ferguson, Supriya Kadam, and Angelika Niemz. I also thank all of the current Niemz Lab members: Eric Houghton, Ono Bacani, and Ilya Tostorukov. I thank all of the former Niemz Lab members and undergraduate/high school summer researchers: Yvonne Klaue, Deepali Shinde, Thomas Carey, Kimberly Chen, Winnie Ding, Stephanie Tan, Peter Vandeventer, Jifeng Qian, Jose Salazar and Barbara Erwin. I thank our collaborators at Claremont BioSolutions (Tanya Ferguson, Robert Doebler, Keith Harrington and Ryan Talbot), as well as our other external collaborators at Seattle Biomedical Research Institute, Program for Appropriate Technology in Health (PATH), and Seattle King County. I thank my parents, Patricia and Henry Roskos, as well as my sister and brother-in-law, Melissa Roskos and Johann Joseph, for their moral support. I also thank Reid Snowden, Hadi Mirmalek-Sani, and Ryan McComb for their support, for proof-reading chapters of my thesis, and for helping me practice my thesis defense presentation. I thank Lindsay Janssen, Academic Coordinator, and Animesh Ray, Director of the PhD Program, for academic support and ensuring all of my program milestones were completed. I thank the members of my PhD committee: Angelika Niemz, Robert Matson, Jim Osborne and Jim Sterling. Finally, I thank the NIH-NIAID for providing funding through grants R01AI076247 and R01AI090831. 


\section{TABLE OF CONTENTS}

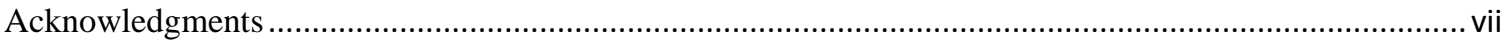

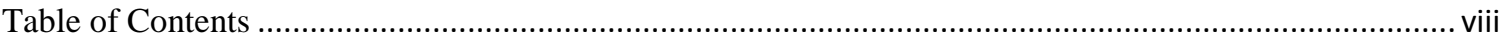

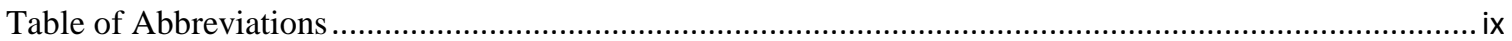

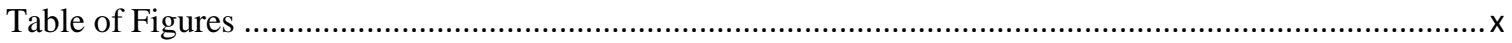

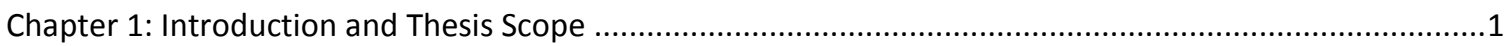

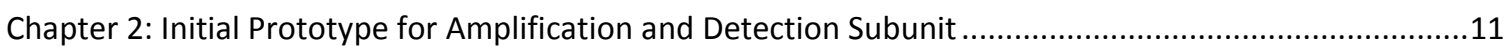

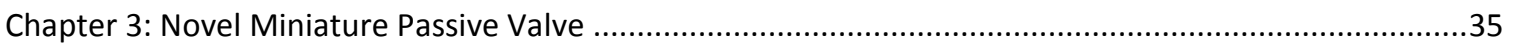

Chapter 4: Refined Prototype for Amplification and Detection Subunit..........................................47

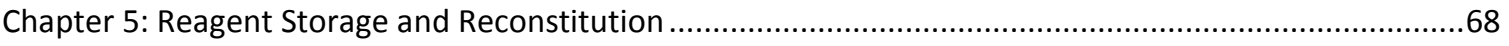

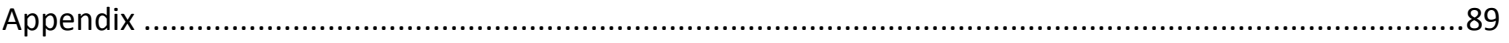

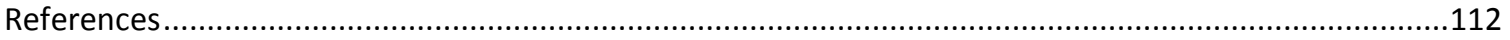


TABLE OF ABBREVIATIONS

\begin{tabular}{|l|l|}
\hline Abbreviation & Full Name \\
\hline ABS & Acrylonitrile butadiene styrene \\
\hline B3 & Backward outer primer \\
\hline Bio-LF & Biotinylated forward loop primer \\
\hline BIP & Backward inner primer \\
\hline BSA & Bovine serum albumin \\
\hline CAD & Computer-aided design \\
\hline CNC & Computer numerical control \\
\hline DIG & Digoxygenin \\
\hline DNA & Deoxyribonucleic acid \\
\hline DST & Drug susceptibility testing \\
\hline ePump & Electrolytic pump \\
\hline EXPAR & Exponential amplification reaction \\
\hline F3 & Forward outer primer \\
\hline FMEA & Failure Mode and Effects Analysis \\
\hline FIP & Forward inner primer \\
\hline IP & Intellectual property \\
\hline KGI & Keck Graduate Institute \\
\hline LAMP & Loop-mediated amplification reaction \\
\hline LB & Backward loop primer \\
\hline LED & Light-emitting diode \\
\hline LOD & Limit of detection \\
\hline MBS & Master of BioScience \\
\hline MDR TB & Multi-drug resistant tuberculosis \\
\hline M.tb & Mycobacterium tuberculosis \\
\hline NAAT & Nucleic acid amplification testing \\
\hline NALF & Nucleic acid lateral flow \\
\hline NTC & No template control \\
\hline PCB & Printed circuit board \\
\hline PCR & Polymerase chain reaction \\
\hline PEEK & Polyether ether ketone \\
\hline POC & Point of care \\
\hline PTFE & Polytetrafluoroethylene \\
\hline RPN & Risk priority number \\
\hline RNA & Ribonucleic acid \\
\hline SOP & Standard operating procedure \\
\hline TB & Tuberculosis \\
\hline USB & Universal serial bus \\
\hline TPP & Target product profile \\
\hline USP & US pharmacopeial convention \\
\hline UV & Ultraviolet \\
\hline & \\
\hline & \\
\hline
\end{tabular}




\section{TABLE OF FIGURES}

Figure 1: Schematic drawing illustrating system operating principle during process execution. Sample is loaded and pushed through a miniaturized bead blender (“Omnivalve”), lysing mycobacteria and eluting DNA into downstream chambers where it reconstitutes lyophilized amplification master-mix materials. The master-mix is then heated, allowing isothermal amplification to occur, and the fluid is then pumped onto lateral flow strips where amplified product can be visually detected by the user.

Figure 2: Conceptual drawing of system components. (a) Cartridge viewed from the top, showing the access ports to the reaction and pump pouches on the underside of the cartridge (indicated in gray outline), and the position of lateral flow strips in anti-parallel orientation. (b) Cartridge viewed from the bottom, showing the reaction pouches with overlaid pump pouches. (c) and (d) Side view of the cartridge on the heater (not to scale), illustrating the operating principle. (c) Master-mix is injected through the septum inlet into the reaction pouch, and targeted DNA sequences are isothermally amplified on top of the temperature-controlled heater surface. (d) Applying current to the electrolytic pump pushes fluid into the pump pouch. The pump pouch expands, thereby compressing the reaction pouch up against the dome-shaped recess in the cartridge, which forces fluid out of the reaction pouch through the one-way check valve and into the lateral flow strip pouch.

Figure 3: Cartridge and instrument. (a) Underside of cartridge, showing two reaction pouches sealed inside two pump pouches on the bottom surface of the bottom card. (b) Top side of cartridge: lateral flow strips are sealed inside the grooves forming the lateral flow strip pouches, and electrolytic chambers are press-fit in place. Not shown: silicone 
foam is placed between the cards, and pump chambers are filled with electrolyte solution to complete the assembly. (c) Small portable prototype heater, which contains the control electronics, thin-film polyimide heater, an aluminum heater surface, and thermal insulation below the heater and around the perimeter of the area where the cartridge is inserted. (d) Heater with a cartridge secured in place (grey lid), prior to test execution. (b) and (d) The red line on the NALF strips represents the dry reagent formulation with colored microspheres dispensed on the conjugate pad.

Figure 4: Conceptual depictions of EXPAR reactions and coupling the amplification products with NALF. (a) First-stage EXPAR, where Trigger X primes and is extended along a template provided in the EXPAR master-mix. (b) Second-stage EXPAR illustrating Trigger $\mathrm{X}$ converting to a reporter oligonucleotide (Reporter Y). (c) NALF coupled to second-stage EXPAR, where the Reporter Y oligonucleotide binds DNAfunctionalized microspheres and DNA hybridized on the nitrocellulose at the test line, resulting in a visible test line. This binding is dependent on the presence of Reporter Y, so the test line will only be present in positive samples. Additional DNA functionalized on the microspheres will bind DNA hybridized on the nitrocellulose at the control line, resulting in a visible line. The binding at the control line is independent of the presence of Reporter Y, and, therefore, acts as an indicator of proper lateral flow function.

Figure 5: (a) Measured temperature of the fluid inside the reaction pouch of a cartridge attached to the heater as a function of time. Filling the pump pouch with liquid improves the thermal transfer from the heater to the reaction fluid, which reaches a stable temperature of $62 \pm 0.1{ }^{\circ} \mathrm{C}$ within approximately ten minutes. Thermal paste applied between the heater surface and pump pouch provides no further improvements. (b) and (c) 
Thermal simulations (COMSOL Multiphysics) of the cartridge on top of the heater, showing horizontal cross-sections within the center of the reaction fluid layer of the cartridge. (b) Uniform heating to the desired temperature $\left(63^{\circ} \mathrm{C}\right)$ is observed using a model with ideal thermal contact between cartridge and heater, with no air gap. (c) Introducing a $150 \mu \mathrm{m}$ thick thermally-resistive layer between cartridge and heater leads to a lower temperature and less uniform heating within the reaction pouch. The crosshatches in the middle of the pump pouches indicate the dimensions of the circular reaction pouches. An air bubble was intentionally introduced into the outlet port of the reaction chamber to simulate air trapped in the reaction pouches after fluid is inserted. Figure 6: Conceptual depiction of NALF detection coupled to LAMP. (a) Amplified master-mix applied to the conjugate pad enables amplicons to interact with colored polystyrene microspheres functionalized with appropriate capture moieties. (b) After migrating along the nitrocellulose membrane, microspheres carrying amplicons are captured at the test line. (c) At the control line, microspheres are captured irrespective of the presence of amplicon.

Figure 7: Amplification results. (a) LAMP based detection of M.tb genomic DNA performed in the cartridge on the instrument: NALF strips of two representative cartridges, after 10 minutes of isothermal amplification, followed by $10 \mathrm{~min}$ for lateral flow detection. (b) LAMP master-mix amplified in the cartridge on the heater, analyzed via gel electrophoresis (Lanes 1 and 2), compared to amplification performed in reaction tubes on a standard heat block (Lanes 3 and 4). Lane 5: DNA molecular weight markers. For (a) and (b), positive (+) reactions show LAMP product starting from 3000 copies of M.tb DNA, and negative (-) reactions show no product since no M.tb DNA was added to 
the reaction. (c) EXPAR based detection of M.tb genomic DNA performed in the cartridge on the instrument: NALF strips of two representative cartridges, after 60 minutes of isothermal amplification, followed by $10 \mathrm{~min}$ for lateral flow detection. Positive (+) reactions contained $6 \times 10^{5}$ copies M.tb DNA, and negative (-) reactions contained no M.tb DNA.

Figure 8: Valve design components: (a) inner rigid core with one end capped and one side port; (b) rigid core with elastomeric sleeve covering side port; (c) encapsulated valve for in-line use.

Figure 9: Different embodiments of the sleeve valve. (a) Stainless steel core tubing with silicone tubing sleeve. (b) PEEK core tubing with cast silicone sleeves, with varying sleeve inner diameters, as indicated in the figure.

Figure 10: Cracking and sustained open pressure for different valve embodiments. (a) Pressure as a function of time for an example steel valve, indicating (i) cracking pressure and (ii) sustained open pressure; (b) Cracking pressure for the two stainless steel valve sets; (c) Cracking pressure for PEEK valve sets 1-5, as a function of silicone sleeve inner diameter

Figure 11: Nucleic acid amplification reactions with (solid lines) and without (dashed lines) autoclaved valve materials incubated in the reaction buffer prior to running the amplification reaction. The valve materials do not inhibit the reaction. A small piece of each valve material, either autoclaved or in its original form, was incubated in LAMP reaction buffer for 60 minutes at $63{ }^{\circ} \mathrm{C}$, with agitation. We then used this buffer solution in setting up LAMP reactions that were subsequently amplified in the presence and absence of the targeted genomic DNA by incubation at $63{ }^{\circ} \mathrm{C}$, with real time fluorescence 
monitoring using an Opticon2 real-time PCR instrument (Bio-rad, Hercules, CA). Blue: LAMP positive reactions containing 3000 copies of target genomic DNA per reaction. Red: LAMP negative control reactions, which did not contain target DNA.

Figure 12: (a) Design concept of the fluidic system of the cartridge. (b) CAD design of the cartridge components including: (i) cartridge core, (ii) spacer ring, (iii) reaction insert, and (iv) bottom casing. (c) Injection molded pieces of the cartridge components.

Figure 13: Steps of cartridge insertion into the designed instrument including (a) insert cartridge datum into locator slots in the instrument housing at an angle and (b) lower the cartridge into the slot and release the spring loaded clip to secure the cartridge.

Figure 14: Fluidic control of reaction pouch filling. Fluid was pumped into the reaction pouch at $3 \mu \mathrm{L} / \mathrm{sec}$ for $91 \mathrm{sec}$, and then the pump was turned off (grey area). The inlet pressure was measured upstream of the inlet valve, in-line with the fluid pumping into the reaction pouch. The pump pouch pressure was measured as a surrogate to indicate the pressure inside the reaction pouch. (a) Inlet pressure increases, then drops, which indicates inlet valve cracking. (b) Inlet and pump pouch pressures build as fluid fills the reaction pouch. The outlet valve does not allow fluid to flow out of the reaction chamber. The small pressure peak presumably indicates dead air trapped in the reaction pouch exiting the chamber through the outlet valve. (c) Maximum peak pressure indicates outlet cracking pressure and the point at which fluid begins flowing out of the reaction chamber. (d) Inlet pumping was stopped, allowing fluid to exit the reaction chamber and pressure inside the reaction pouch to equilibrate.

Figure 15: Pressure measurement in the upstream channel during injection of $150 \mu \mathrm{L}$ reaction buffer from the sample inlet port into the reaction insert. (a) The barrier vents the 
dead air ahead of the fluid until the fluid fills the upstream channel. No air is injected into the reaction pouch. (b) The venting barrier closes as liquid fills the area underneath the barrier. Pressure builds in the upstream fluidic channel until the inlet valve opens. (c) The fluid is pumped into the reaction pouch through the inlet valve. The channel pressurizes due to the pressure exerted by the filled and closed pump pouch. (d) After approximately $100 \mu \mathrm{L}$ liquid is injected into the reaction pouch, the gas behind the injected volume is vented through the venting barrier and the remainder of the volume is trapped in the vented portion. No air is injected into the reaction pouch.

Figure 16: Lateral flow strip chamber testbed. (a) Empty lateral flow strip chambers, showing embedded hydrophobic barriers (blue). (b) Lateral flow strip chambers with lateral flow strips sealed by polycarbonate film. The red line on the conjugate pads is the lateral flow conjugate that has been dispensed and dried onto the conjugate pad.

Figure 17: Temperature of the fluid inside the reaction pouch of a cartridge attached to the heater as a function of time. The fluid reaches a stable temperature within approximately ten minutes.

Figure 18: Demonstration of fluidic process in cartridge on instrument. (a) Cartridge attached to instrument, with reaction buffer (colored blue) injected into storage chamber. (b) Instrument LEDs indicate function of heater (red LED) and ePumps (blue LEDs). (c) Lateral flow detection of reaction buffer spiked with synthetic DNA product pumped out of the reaction pouch onto the lateral flow strips in the cartridge.

Figure 19: Detection of M.tb genomic DNA through 60 minutes of isothermal LAMP in the cartridge on the instrument, verified via gel electrophoresis of amplified master-mix removed from the cartridge after the reaction. Amplified product from both reaction 
pouches (lanes 3 and 4) generated a similar pattern as the positive control reactions performed in tubes on a standard heater (lanes 5 and 6), indicating that desired amplification had occurred in the cartridge. All positive reactions contained 3000 copies $M . t b$ genomic DNA. No products were generated in negative control reactions (lanes 7 and 8) that did not contain M.tb DNA. Lane 1 contained $1 \mathrm{~kb}$ molecular weight ladder and lane 2 was empty.

Figure 20: Lyophilized pellet storage and reconstitution chamber as originally designed. (a) Reconstitution of pellet (red arrow) in blue colored water. (b) Depiction of reconstitution, illustrating the pellet wicking the initial fluid front to the bottom of conical chamber and pumping further downstream, while introducing an air bubble in the fluid stream separating the initial fluid front (ii) from the remaining fluid (i).

Figure 21: Original system overview diagram. Red area highlights the fluidics interface between sample preparation and amplification and detection, which needs to store stabilized master-mix reagents until they can be reconstituted by purified sample preparation eluate and pumped into the reaction pouch chamber. The initial design incorporated lyophilization to stabilize the master-mix reagents.

Figure 22: Fluidic system overview. (a) Top view: purified sample enters the chamber, first absorbing into the lyophilized master-mix material matrix, and then filling the chamber completely. (b) Side view: after a short incubation period to allow the reagents to diffuse, the reconstituted reaction master-mix is pumped through the outlet.

Figure 23: Investigation of different reconstitution chamber geometries. For each case, showing (i) empty chamber, (ii) partially filled chamber, and (iii) chamber after as much fluid could be emptied as possible. (a) Ellipse without any structures. (b) Ellipse with 
structure in front of outlet intended to catch any air trapped in the chamber. (c) Ellipse with chevron structures intended to enhance mixing in the chamber. (d) Ellipse with cylindrical pillars intended to enhance mixing in the chamber. All structures inhibited emptying of the chambers, leaving large dead volumes of unrecoverable fluid in the chambers. The empty ellipse shape (a) had the largest fluid recovery.

Figure 24: Reconstitution chambers with integrated venting barriers. (a) Reconstitution chamber with commercially-available hydrophobic barrier integrated into chamber. (b) Reconstitution chamber with integrated hydrophobic barrier and microfiber material, which had a higher percentage of fluid that could not be recovered from this chamber design. (c) Polypropylene-backed PTFE membrane material heat-sealed to an isolated venting chamber downstream of the reconstitution chamber.

Figure 25: Fluidic interface testbed demonstrating the reconstitution of dried food coloring. (a) Top side of fluidic interface testbed showing one of the two fluidic module testbeds, including the venting chamber (white) and the reconstitution chamber, with orange food coloring dried onto the CF1 microfiber material. (b) Reconstitution chamber filled and reconstituting the orange food coloring, with fluid beginning to pump through the fluid conduit on the underside of the testbed from the reconstitution chamber to the venting chamber. (c) Bottom side of fluidic interface testbed, showing polypropylene reaction insert with empty reaction and pump pouches. (d) Reaction pouch with orange colored water inside after reconstituting the food coloring and pumping through the oneway valve into the reaction pouch chamber.

Figure 26: Reconstitution of dried LAMP master-mix reagents. Black solid line is reconstituted LAMP master-mix spiked with $M . t b$ genomic DNA (positive). Black 
dashed line is reconstituted LAMP master-mix without M.tb genomic DNA (negative). Amplification of negative sample is most likely due to lab amplicon contamination affecting the reagent drying process.

Figure 27: Amplification of reconstituted master-mix materials. (a) Amplification results from two samples reconstituted in the fluidics testbed, with M.tb genomic DNA spiked into the reconstitution buffer. Testbed \#1 shows amplification while Testbed \#2 does not. (b) Amplification results from samples reconstituted in standard tubes and amplified on a bench-top thermocycler. The reconstituted NTC sample did not contain M.tb genomic DNA but does show amplification, likely due to amplicon contamination of lab areas. 


\section{CHAPTER 1: INTRODUCTION AND THESIS SCOPE}

Nucleic acid amplification testing (NAAT) can be used to diagnose infectious diseases by identifying the pathogen's genetic material. ${ }^{1,2}$ NAAT is typically performed in centralized laboratories by highly-trained personnel on large, complex, expensive equipment, which is not ideal for applications that require a rapid answer to facilitate treatment and improve patient outcomes. ${ }^{3-5}$ In developing countries, the diagnosis of endemic infectious diseases using NAAT in central laboratories is further hampered by lack of suitable facilities and trained personnel, and additional logistical challenges. ${ }^{6-8}$

\section{Point of Care Technologies}

Technologies that enable point-of-care (POC) diagnosis of active tuberculosis (TB) could greatly benefit the treatment and containment of the disease, ${ }^{9}$ which in 2011 caused 8.7 million incident cases, resulting in 1.4 million deaths, ${ }^{10}$ primarily in high TB-burden regions of Asia, Eastern Europe, and Sub-Saharan Africa. Rapidly identifying individuals with active infectious TB in the field is of critical importance to curb transmission and reduce morbidity and mortality. ${ }^{11,12}$ Smear microscopy is commonly used to diagnose active pulmonary $\mathrm{TB}$, but requires multiple sputum samples, tedious processing, and often has low sensitivity. ${ }^{6}$ Culture methods, the current gold standard for sensitive TB diagnosis and drug susceptibility testing (DST), ${ }^{12}$ typically deliver results in $2-8$ weeks, involve significant biohazard concerns, and, therefore, require expensive biosafety level 3 laboratory infrastructure and trained personnel. By contrast, NAAT enables sensitive and specific TB diagnosis and genotypic drug resistance testing, producing accurate results in less than 1 day, ${ }^{13,14}$ not accounting for the time required for sample transfer to a central laboratory, and result dissemination to the care provider. POC NAAT eliminates these 
often substantial delays, enables testing and treatment initiation in the same visit, and can significantly improve access of TB patients to proper diagnostic methods.

The GeneXpert (Cepheid, Sunnyvale, CA) exemplifies the move of NAAT towards the point of care. ${ }^{14}$ This fully-automated and integrated bench-top system can perform sample preparation, nucleic acid amplification via polymerase chain reaction (PCR), and multi-channel real-time fluorescence detection in less than 2 hours. ${ }^{15-17}$ The World Health Organization (WHO) has endorsed the GeneXpert MTB-Rif test to identify multi-drug resistant tuberculosis (MDR TB). However, the size, cost, and maintenance requirements of the GeneXpert instrument may impede broad-scale implementation in resource-limited, high-burden countries. ${ }^{18}$ Other PCR-based fully- or partially-integrated NAAT systems for infectious disease diagnosis are in development or on the market, ${ }^{19-22}$ but are relatively expensive systems due to the complexity associated with thermocycling and real-time fluorescence detection. Isothermal NAAT requires a single reaction temperature, and, therefore, utilizes simplified instrumentation compared to real-time PCR. In recent years, significant progress has been made in automating isothermal amplification methods within a miniaturized format. ${ }^{23-26}$ Several isothermal NAAT assays and systems have entered the US market for infectious disease diagnosis in moderate complexity clinical laboratories, ${ }^{27-29}$ with varying levels of process integration, system complexity, and ease of use.

Several groups have developed sample-in to answer-out lab-on-a-chip systems for nucleic acid testing. ${ }^{24-26,30-33}$ Microfluidic systems for PCR amplification with real-time fluorescence detection $^{34,35}$ have been commercialized as part of the Biomark ${ }^{\mathrm{TM}}$ (Fluidigm, San Francisco, CA) and BD MAX ${ }^{\mathrm{TM}}$ (Becton Dickinson, Franklin Lakes, NJ) systems, 
which both comprise large bench-top instruments, with sample preparation performed off-chip using standard methods. Microfluidic PCR systems such as the Biomark ${ }^{\mathrm{TM}}$ have significant potential for high-end applications. However, to enable fully-automated sample-to-answer nucleic acid testing at the point of care using microfluidic PCR, challenges have to be overcome related to overall complexity and cost of both the chip and required instrumentation. NAAT for infectious diseases further requires large sample input volumes to reach the required limit of detection (LOD). In the case of tuberculosis, typical sputum sample input volumes range from $100 \mu \mathrm{L}^{13,36}$ to several $\mathrm{mL}{ }^{14}$ Microfluidic systems require a much smaller sample input volume, while mesofluidic systems are ideal for processing samples in this volume range. In addition, real-time PCR requires thermocycling plus fluorescence optics for detection, which is difficult to implement in a compact, low-cost, and robust instrument for use in low-resource settings. Isothermal NAAT requires a single reaction temperature, and, therefore, utilizes simplified instrumentation, compared to PCR. One such method, the loop-mediated amplification (LAMP) reaction, uses four to six primers that recognize multiple sequences in the target DNA. ${ }^{37}$ The reaction generates concatenated DNA products with high molecular weight and extensive secondary structure. A LAMP assay targeting the gyrB gene in Mycobacterium tuberculosis (M.tb) genomic DNA,${ }^{38}$ coupled to visual endpoint detection based on pyrophosphate precipitation, has been applied to TB diagnosis in low-resource settings. ${ }^{39,40}$ However, the method is performed manually in multiple steps that require additional instruments; the turbidimetric fluorescence-based readout requires a UV light source for excitation, and this detection method may give an ambiguous result at low target copy numbers. 


\section{Nucleic Acid Lateral Flow}

Lateral flow devices, as exemplified by pregnancy tests, are well-established for POC diagnostics, can be manufactured inexpensively in large quantities, rely on passive fluidics, and provide a clear visual readout with no additional instruments required. Nucleic Acid Lateral Flow (NALF) has been coupled to $\mathrm{PCR}^{41}$ and to many isothermal methods. ${ }^{42-46}$ NALF involves either antibody-dependent or antibody-independent configurations for endpoint detection of a reaction, with the sensitivity contingent upon upstream amplification. Antibody-dependent NALF has been used to detect LAMP products ${ }^{46-49}$ by incorporating antigenic labels or haptens into the amplicon. NALF detection is then performed using an antibody specific to the label. Antibody-independent NALF uses direct hybridization of unlabeled target amplicons to colored oligonucleotidefunctionalized micro-particles and immobilized oligonucleotides on the test strip membrane. ${ }^{41,50}$

Lateral flow devices are well-established for POC diagnostics, can be manufactured inexpensively in large quantities, rely on passive fluidics, and provide a clear visual readout with no additional instruments required. NAAT systems for infectious disease diagnosis commercialized by BioHelix ${ }^{29}$ and $\mathrm{USTAR}^{42}$ use a NALF cassette to analyze the amplified master-mix. This NALF cassette completely contains the amplified master-mix to avoid amplicon carry-over contamination, which is a major challenge in NAAT. However, in these systems, NALF detection is performed as a separate manual step, after isothermal amplification has been executed on a standard heat block. 


\section{Cartridge Fluidics}

To integrate isothermal DNA amplification with NALF detection in one device requires a system that can heat the master-mix at a fixed temperature for a pre-determined time, and then pump the amplified master-mix onto the lateral flow strip. While isothermal heating can be readily accommodated in a compact, inexpensive instrument, fluid handling on disposable chips or inside cartridges typically requires bulky and complex positive displacement pumping systems. For example, the Cepheid GeneXpert ${ }^{16}$ uses a mechanically actuated piston with a rotating valve. Other systems, including the CARD (Chemistry and Reagent Device, Rheonix, Ithaca, NY), the LIAT (lab in a tube, IQuum, Marlborough, MA), the Razor and Film Array systems (Idaho Technologies, Salt Lake City, Utah), and the Portrait analyzer (Great Basin Scientific, Salt Lake City, Utah), utilize pneumatic or mechanical actuation for pumping. ${ }^{21,22,28,51,52}$ Alternatively, electrolysis provides an inexpensive mechanism to pump fluids by using hydrogen and oxygen gases generated by water electrolysis to exert pressure on a downstream fluid. ${ }^{53}$ Electrolytic pumping has been used for automated NAAT inside microfluidic devices as developed by Motorola ${ }^{54}$ and Combimatrix. ${ }^{55,56}$

\section{System Overview}

The overall goal of this project is to develop a fully-integrated NAAT device to diagnose infectious diseases, such as TB, at the point of care in low-resource settings. The envisioned device will be a handheld, closed system that is inexpensive and easy to use. Ultimately, this device will combine sample preparation, isothermal DNA amplification and lateral flow detection into a single sample-in to answer-out platform. This scheme 
comprises a disposable cartridge that mates with a reusable handheld electronic device.

This disposable cartridge will contain all necessary reagents, and will not require refrigeration, including during shipping and storage. The handheld electronics device will be low-power to enable eventual battery operation, making the entire system fielddeployable. To be appropriate for low-resource settings, the cost of goods and manufacturing of our system needs to be very low, ideally less than $\$ 10$ per cartridge and less than $\$ 100$ for the handheld device. The total assay time, including sample preparation, will be approximately 60-90 minutes. In addition, we are designing the system to be modular so that multiple cartridges can be run simultaneously by adjusting the handheld electronics device to accommodate more than one cartridge.

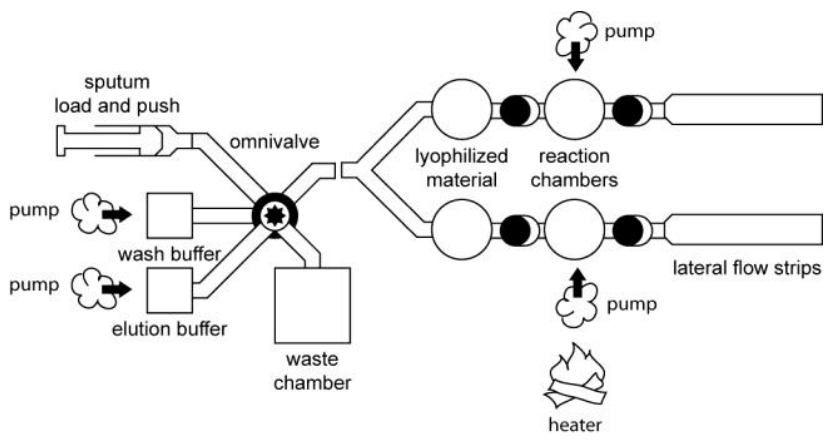

Figure 1: Schematic drawing illustrating system operating principle during process execution. Sample is loaded and pushed through a miniaturized bead blender (“Omnivalve"), lysing mycobacteria and eluting DNA into downstream chambers where it reconstitutes lyophilized amplification master-mix materials. The master-mix is then heated, allowing isothermal amplification to occur, and the fluid is then pumped onto lateral flow strips where amplified product can be visually detected by the user. 
The overall system concept is illustrated in Figure 1. The patient sputum sample will be collected in a separate apparatus that will contain disinfecting and liquefying reagents. This sample collection device will then be attached directly to the inlet port of the cartridge. The sample will be pushed through a proprietary miniature bead blender which has been enclosed inside an active valve (Omnivalve, Claremont BioSolutions, Upland, CA). While the sample is being pushed through the valve to waste, the blender motor agitates the beads inside the blender chamber at 30,000 RPM, which facilitates effective mechanical lysis of the mycobacteria. ${ }^{57}$ Furthermore, the eluted DNA in the sample is captured on the beads inside the blender. The active valve is then turned to connect the blender chamber with the wash buffer reservoir. An electrolytic pump then pushes wash buffer through the blender and into waste to remove contaminants, but retaining the DNA bound to the beads inside the blender. After the wash buffer reservoir has emptied, an additional $1 \mathrm{~mL}$ of air is passed through the blender chamber, in order to fully expel the wash buffer from the blender chamber. Initial studies have shown that this protocol reduces the amount of wash buffer propogated downstream into the reaction master-mix, which is desirable. Following the wash step, the active valve is then turned again, to connect the blender chamber to the elution buffer reservoir. Another electrolytic pump is utilized to fill the blender chamber with elution buffer, and then the valve is turned again to connect to the downstream fluidic pathways. The blender is then turned on again, eluting the DNA off the bead matrix and into the buffer solution inside the blender chamber. Since the valve already positioned to connect the blender chamber to the downstream cartridge fluidics, a third electrolytic pump is activated to force the elution fluid out of the blender chamber and into two downstream chambers where it 
reconstitutes lyophilized amplification reaction reagents. After reconstitution, the third electrolytic pump is activated again to push the reconstituted reaction master-mix through one-way passive valves into the amplification reaction chambers, where the reaction mixture is heated and isothermal DNA amplification can occur. During this process, the upstream active valve is turned to the completely closed position, to separate the upstream sample preparation fluidics from the downstream amplification and detection areas. After amplification, two new electrolytic pumps (one for each reaction chamber) push the amplified master-mix through one-way passive valves onto lateral flow strips, where amplified product can be visually detected by the user as colored lines on the lateral flow strips. The entire process from sample preparation through amplification and detection in the cartridge is anticipated to be completed in approximately 60-90 minutes.

During the design and development of this integrated cartridge, we decided to initially divide the fully integrated system into two subsystems: one sample preparation module and one amplification and detection module.

\section{Thesis Scope}

The focus of this thesis is the development of the amplification and detection module, which can execute isothermal amplification coupled to lateral flow detection with required reagents stored on-board, as well as automated and integrated pumping, heating, and timing mechanisms. In Chapter 2, we describe the development of an early prototype for a disposable, pouch-based cartridge to automate amplification and detection, in conjunction with a compact, inexpensive heater that contains low power electronics. In

Chapter 3, we describe a novel disposable, miniature check valve design that is suitable for large-scale manufacturing. In Chapter 4, we describe a refined amplification and 
detection cartridge with integrated electrolytic pumps and check valves (as described in Chapter 3), plus an improved handheld unit that automates heating, timing and pumping for amplification and detection process execution. In Chapter 5, we discuss the fluid interface that will integrate the amplification and detection module with the sample preparation module into one fully-integrated cartridge, and the development of the onboard lyophilized master-mix reagent storage and reconstitution.

This work involves a collaborative effort, including KGI engineers (Drs. Anna Hickerson and Hsiang-Wei Lu), KGI scientists (Drs. Supriya Kadam, Eric Houghton, Deepali Shinde, and Yvonne Klaue), and summer undergraduate or high school research students (Thomas Carey, Kimberly Chen, Winnie Ding and Stephanie Tan), as well as input from Claremont BioSolutions engineers (Dr. Robert Doebler, Ryan Talbot and Keith Harrington) and scientist (Tanya Ferguson, also from KGI). My contribution to the work presented in each chapter is highlighted below (Table 1). An organization chart is included in Appendix B. 
Table 1: My individual contributions throughout this thesis project.

\begin{tabular}{|c|c|c|}
\hline Chapter & Team Collaborators & My Individual Contributions \\
\hline $\begin{array}{l}\text { 2: Initial } \\
\text { Prototype for } \\
\text { Amplification } \\
\text { and Detection } \\
\text { Subunit }\end{array}$ & $\begin{array}{l}\text { Anna Hickerson, Hsiang- } \\
\text { Wei Lu, Tanya M. } \\
\text { Ferguson, Deepali N. } \\
\text { Shinde, Yvonne Klaue }\end{array}$ & $\begin{array}{l}\text { Participated in original design of cartridge and heater; } \\
\text { Developed pouch fabrication method; } \\
\text { Developed lateral flow strip integration into pouches; } \\
\text { Refined electrolytic pumps; } \\
\text { Verified cartridge materials-assay compatibility; } \\
\text { Selected appropriate plastic for injection molding; } \\
\text { Performed thermal mapping to characterize thermal control } \\
\text { in the cartridge; } \\
\text { Manufactured and assembled cartridges; } \\
\text { Assembled and calibrated heater units; } \\
\text { Established clean manufacturing methods; } \\
\text { Conducted all isothermal nucleic acid amplification } \\
\text { experiments in the cartridges on the heaters }\end{array}$ \\
\hline $\begin{array}{l}\text { 3: Novel } \\
\text { Miniature } \\
\text { Passive Valve }\end{array}$ & $\begin{array}{l}\text { Anna Hickerson, Hsiang- } \\
\text { Wei Lu, Thomas Carey }\end{array}$ & $\begin{array}{l}\text { PEEK valve assembly and testing; } \\
\text { Developed autoclaving protocol for decontamination; } \\
\text { Validated valve material-assay compatibility; } \\
\text { Performed statistical analyses on all valve data sets; } \\
\text { Performed background literature and IP research }\end{array}$ \\
\hline $\begin{array}{l}\text { 4: Refined } \\
\text { Prototype for } \\
\text { Amplification } \\
\text { and Detection } \\
\text { Subunit }\end{array}$ & $\begin{array}{l}\text { Anna Hickerson, Hsiang- } \\
\text { Wei Lu, Thomas Carey }\end{array}$ & $\begin{array}{l}\text { Participated in iterative redesign of refined cartridge and } \\
\text { instrument; } \\
\text { Characterized polycarbonate thermal bonding; } \\
\text { Redesigned lateral flow chambers for proper fluidic } \\
\text { performance; } \\
\text { Manufactured components and assembled cartridges; } \\
\text { Participated in clean-manufacturing process development; } \\
\text { Participated in fluidic characterization of cartridge; } \\
\text { Verified assay compatibility of new cartridge components } \\
\text { and materials; } \\
\text { Conducted all LAMP assay tests within the cartridges, } \\
\text { including external verification of LAMP product } \\
\text { formation via gel electrophoresis }\end{array}$ \\
\hline $\begin{array}{l}\text { 5: Reagent } \\
\text { Stabilization } \\
\text { and } \\
\text { Reconstitution }\end{array}$ & $\begin{array}{l}\text { Hsiang-Wei Lu, Supriya } \\
\text { Kadam, Eric Houghton }\end{array}$ & $\begin{array}{l}\text { Designed and tested reagent storage and reconstitution fluidic } \\
\text { interface module; } \\
\text { Characterized and selected microfiber material; } \\
\text { Participated in the development of the reagent drying } \\
\text { process; } \\
\text { Designed and manufactured fluidic testbeds for } \\
\text { characterization of master-mix reagent reconstitution and } \\
\text { amplification; } \\
\text { Conducted all reconstitution and amplification tests on fluidic } \\
\text { testbeds }\end{array}$ \\
\hline
\end{tabular}




\section{CHAPTER 2: INITIAL PROTOTYPE FOR AMPLIFICATION AND DETECTION SUBUNIT}

\section{Introduction}

The key functional components of the amplificiation and detection subunit include a double pouch system with an inner reaction pouch enclosed by a pump pouch, which is part of the pumping mechanism to move fluid out of the reaction pouch. The reaction pouch requires an inlet to introduce master-mix into the pouch, and an outlet that interfaces the lateral flow strip for detection. The pump pouch is connected to an electrolytic pump. The system is heated to a controlled and constant temperature for the duration of the isothermal amplification reaction. While designing and demonstrating functionality of these key components, we developed an intial disposable cartridge prototype, in conjunction with a handheld heater prototype. We have demonstrated as proof of principle the amplification and detection of Mycobacterium tuberculosis (M.tb) genomic DNA in the cartridge, using either Loop Mediated Amplification (LAMP) or the Exponential Amplification Reaction (EXPAR), both coupled to Nucleic Acid Lateral Flow (NALF) detection.

\section{Experimental}

System Design and Operating Concept: Our system design, illustrated in Figure 2, allows two samples to be tested simultaneously. The disposable cartridge contains on its top side two lateral flow strips in anti-parallel orientation, two septum inlets through which master-mix is injected, and two attachment ports for electrolytic pumps (Figure 2a). Two reaction pouches are attached to the bottom side of the cartridge (Figure 2b), accessible through septum inlets and outlets containing passive, one-way check valves that lead to 
the lateral flow strips. The bottom side of the cartridge also contains two pump pouches sealed on top of the reaction pouches, which are connected to the electrolysis chamber ports. The electrolysis chambers and the pump pouches are filled with electrolyte solution. During process execution, an empty cartridge is attached to the handheld, nondisposable heating and electronics unit, where the cartridge is pre-heated to the reaction temperature. After the cartridge has reached the appropriate operating temperature, reaction master-mix is injected from the top (Figure 2c), through the septum inlet port, into the reaction pouch, which initiates isothermal DNA amplification. Once the reaction is completed, current is applied to the electrodes (Figure 2d) and the pump pouch is pressurized with gas produced via electrolysis. The pressurized pump pouch pushes the fluid within the reaction pouch up through the outlet port and onto the lateral flow strip. The reaction mixture migrates along the strip through passive capillary action, wicking across test and control areas of the strip where colored microspheres can bind, producing a visual readout. The lateral flow strips are also sealed in pouches, ensuring that all fluids remain sealed within the disposable cartridge, eliminating carry-over contamination of amplicons. 
(a)
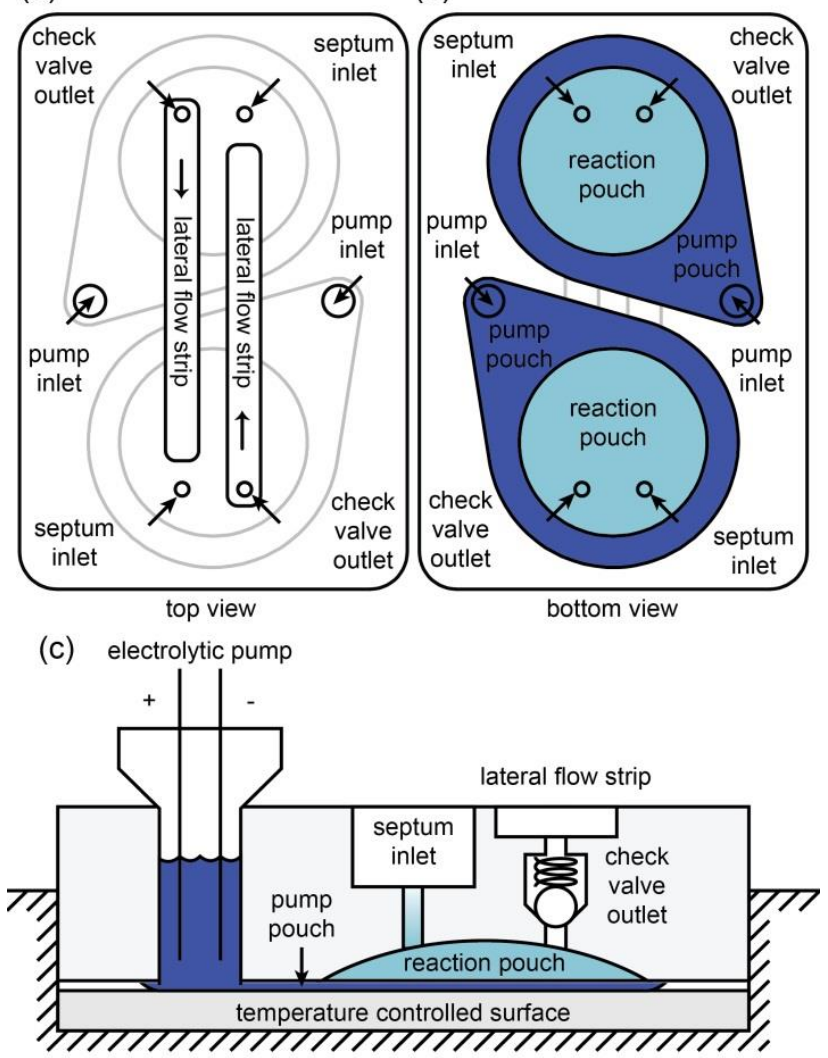

(d) electrolytic pump

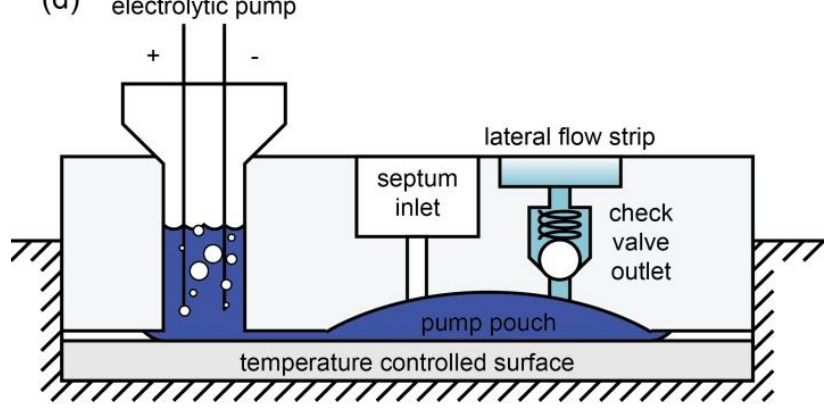

Figure 2: Conceptual drawing of system components. (a) Cartridge viewed from the top, showing the access ports to the reaction and pump pouches on the underside of the cartridge (indicated in gray outline), and the position of lateral flow strips in anti-parallel orientation. (b) Cartridge viewed from the bottom, showing the reaction pouches with overlaid pump pouches. (c) and (d) Side view of the cartridge on the heater (not to scale), illustrating the operating principle. (c) Master-mix is injected through the septum inlet into the reaction pouch, and targeted DNA sequences are isothermally amplified on top of 
the temperature-controlled heater surface. (d) Applying current to the electrolytic pump pushes fluid into the pump pouch. The pump pouch expands, thereby compressing the reaction pouch up against the dome-shaped recess in the cartridge, which forces fluid out of the reaction pouch through the one-way check valve and into the lateral flow strip pouch.

Cartridge Fabrication: The cartridge uses low-cost components and manufacturing techniques to meet the cost constraints of POC testing performed in low-resource settings. The main cartridge components are manufactured using injection molding and thermal bonding, both of which are inexpensive and scalable techniques.

The body of the cartridge consists of two polypropylene cards, which were injection molded in-house from USP Class VI polypropylene pellets (Pro-fax SR256M, PolyOne, Inc., Rancho Cucamonga, CA) using a G-100T injection molding press from Morgan Industries (Long Beach, CA). The cards include hollow protrusions in matching locations, which can be joined to form fluid conduits that allow fluid to pass from the bottom surface of the bottom card to the top surface of the top card. One-way passive ball-and-spring valves (CCPI2510014S, the Lee Company, Westbrook, CT) were pressed into the hollow protrusions of the bottom card, leading from the reaction pouches to the lateral flow strip pouches (Figure 2a, check valve outlet). To create a silicone inlet septum, short pieces of silicone cord (McMaster-Carr, Santa Fe Springs, CA) were inserted into the hollow protrusions on the top card, serving as inlets into the reaction pouches (Figure 2a, septum inlet). Next, 0.005 inch thick flexible polypropylene film (Qosina, Edgewood, NY) was heat-sealed onto the bottom card to create two reaction 
pouches overlaid by two pump pouches (Figure 3a). Lateral flow strips were inserted into grooves on the top card, and polypropylene film was heat-sealed onto the card to create two lateral flow strip pouches (Figure 3b). Heat-sealing was performed using custom-cut aluminum dies to produce pouches of various geometries, attached to a custom-built heat press with a temperature-controlled aluminum block mounted to an arbor press for safety and leverage. After attaching the pouches, the two cards were pressed together, creating a snap-fit seal and leak-proof fluid conduits between the two cards. We then inserted silicone foam insulation ( $85925 \mathrm{~K} 115$, McMaster) cut to the appropriate shape between the top and bottom cards. To complete the cartridges, two large reservoirs (Qosina) were press fit into the fluid conduits that provide access to the pump pouch (Figure 2a, pump inlet), thus forming the electrolyte chambers on the top of the cartridge (Figure 3b). We used standard 18 gauge stainless steel syringe needle tips to fill the chamber and pump pouch with $1 \mathrm{M}$ aqueous $\mathrm{Na}_{2} \mathrm{SO}_{4}$ electrolyte solution through the septum on top of the reservoir chamber. The two needles were then capped closed, but were left in the septum, to be used as electrodes for electrolytic pumping.
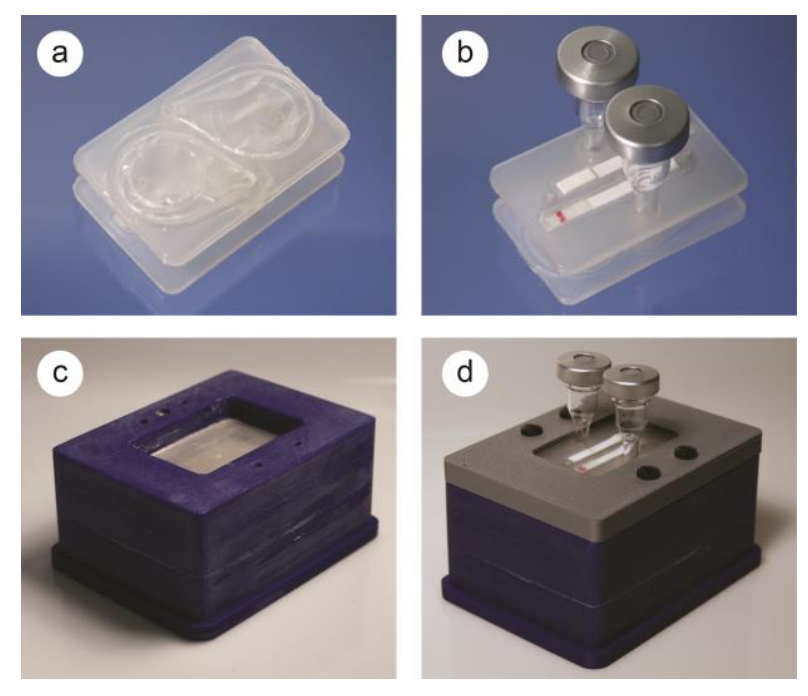
Figure 3: Cartridge and instrument. (a) Underside of cartridge, showing two reaction pouches sealed inside two pump pouches on the bottom surface of the bottom card. (b) Top side of cartridge: lateral flow strips are sealed inside the grooves forming the lateral flow strip pouches, and electrolytic chambers are press-fit in place. Not shown: silicone foam is placed between the cards, and pump chambers are filled with electrolyte solution to complete the assembly. (c) Small portable prototype heater, which contains the control electronics, thin-film polyimide heater, an aluminum heater surface, and thermal insulation below the heater and around the perimeter of the area where the cartridge is inserted. (d) Heater with a cartridge secured in place (grey lid), prior to test execution. (b) and (d) The red line on the NALF strips represents the dry reagent formulation with colored microspheres dispensed on the conjugate pad.

Portable Heater Fabrication: We designed and fabricated a reusable handheld instrument (Figure 3c) to apply a precise and controlled temperature to the reaction fluid within the disposable cartridge (Figure 3d). The set-point of the heater can be adjusted to reach the temperature required for a particular isothermal reaction, e.g. $63{ }^{\circ} \mathrm{C}$ for LAMP and $55^{\circ} \mathrm{C}$ for EXPAR. The chassis of the heater instrument was printed with acrylonitrile butadiene styrene (ABS) using a Dimension SST 768 fused deposition modeling machine. Thinfilm polyimide heaters (Omega) were attached to a 1/8th inch thick aluminum plate to provide evenly-distributed heating. Polyurethane foam sealant (Dow Chemical), applied to the surrounding area, minimizes the power required to reach and maintain the required temperature for isothermal amplification. We controlled the temperature using a custom printed circuit board (PCB) to adjust the power to the heaters based on feedback from a 
thermistor embedded near the center of the top surface of the aluminum plate. The instrument was powered by a $15 \mathrm{~V}$ wall transformer, using $\sim 8 \mathrm{~W}$ during the initial $\sim 3$ minutes heating ramp, and $\sim 0.5 \mathrm{~W}$ to maintain the temperature.

Thermal Control Testing and Modeling: Precise temperature control is essential for this system, as the amplification reaction kinetics are related to assay fluid temperature. We measured the temperature of the heater surface as a function of time using a DirecTemp (Redfish Sensors, Meridian, ID) universal serial bus (USB) thermistor-based sensor probe. To directly measure the temperature of the fluid in the reaction pouches as a function of time, we inserted a thermocouple (5TC-TT-K-36-36, Omega, Stamford, CT) into the inlet of a reaction chamber filled with water while the cartridge was attached to the heater. We measured the cartridges under three different conditions: with empty pump pouches (no electrolyte solution), with pump pouches filled with electrolyte solution, and with pump pouches filled with electrolyte and a thin layer of thermal paste (OmegaTherm "201", Omega, Stamford, CT) applied to the underside of the cartridge on the outside of the pump pouches.

We modeled the three-dimensional temperature profile within the system as a function of three factors: the overall system design, the choice of materials, and the thermal contact between cartridge and heater. Thermal simulations were performed using COMSOL Multiphysics (Burlington, MA), based on the three-dimensional cartridge and instrument geometry imported from Solidworks (Glendora, CA). The model treats the thin-film heater as a two-dimensional heat source on the bottom of the aluminum heater plate, with a specified output power. The heat conducts through the interior of the 
cartridge and dissipates to the room-temperature environment by convection to still air through the top surface of the cartridge, or through insulating material on the sides of the cartridge. Convection through still air is imposed using a natural convection boundary with a Dirichlet heat flux based on: $N V=(5 \mathrm{~W}) /\left(\mathrm{m}^{2} \mathrm{~K}\right)$. We modeled the non-ideal thermal contact between the cartridge and the heating surface by incorporating a thermally resistive layer.

Isothermal Amplification Reactions: For this proof of principle study, we implemented two isothermal DNA amplification reactions in the device to detect $M . t b$ genomic DNA, coupled with NALF visual endpoint detection: an established and clinically validated LAMP reaction, and an in-house developed EXPAR reaction.

For isothermal LAMP targeting the $M . t b$ gyrB genomic region, we modified primer sequences (Table 2Table ) and reaction conditions from Iwamoto et al. ${ }^{38}$ to work in our system with lateral flow detection of amplified products. The $100 \mu \mathrm{L}$ LAMP master-mix contained $20 \mathrm{mM}$ Tris- $\mathrm{HCl}, \mathrm{pH} 7.9,15 \mathrm{mM}$ ammonium sulfate, $30 \mathrm{mM}$ potassium chloride, $0.005 \%$ Triton $\mathrm{X}-100,2 \mathrm{mM}$ magnesium chloride, $2 \mathrm{mM}$ magnesium sulfate, $400 \mu \mathrm{M}$ each dNTP (Fermentas, Glen Burnie, Maryland), 1 M betaine, 200 $\mu \mathrm{g} / \mathrm{mL}$ bovine serum albumin, 2.4 $\mu \mathrm{M}$ Forward Inner Primer (FIP), 1.6 $\mu \mathrm{M}$ Backward Inner Primer (BIP, DIG-labeled), $0.2 \mu \mathrm{M}$ of each outer primer (F3 and B3), $0.4 \mu \mathrm{M}$ biotinylated Forward Loop Primer (bio-LF), $0.8 \mu \mathrm{M}$ Backward Loop Primer (LB), and 20 U of Bst DNA Polymerase large fragment (New England BioLabs, Ipswich, MA). Each reaction further contained $10 \mathrm{ng}$ human genomic DNA (Promega, Madison, WI) as general background. Positive reactions contained 3000 copies of M.tb H37Ra genomic 
DNA (ATCC, Manassas, VA) as the template, which was omitted in the No Template Control (NTC) negative reactions. To characterize the assay performance, LAMP reactions were amplified on a thermocycler set to $63{ }^{\circ} \mathrm{C}$ for the same time period as was used for amplification in the cartridges. Amplified LAMP master-mix was then analyzed using gel electrophoresis on a $2 \%$ eGel (Invitrogen, Life Technologies, Carlsbad, CA).

Table 2: Oligonucleotide Sequences for LAMP Amplification and NALF Detection

\begin{tabular}{ll}
\hline Name & Sequences \\
\hline Forward Outer Primer & GCGATATCTGGTGGTCTGC \\
Backward Outer Primer & CCGTGGTTTCGAAAACAGC \\
Forward Inner Primer & AGACCACTCGTACCCGTCGCCGGTGGTTAACGCGCTAT \\
Backward Inner Primer & DIG-sp9-ATGAGAAGTCGGAACCCCTGGGACCGTTGACCCCGTCTTC \\
Forward Loop Primer & biot-TEG-sp18-TTGATCTCGACTTCGAGCC \\
Backward Loop Primer & CCTCAAGCAAGGGGCG \\
NALF Conjugate Probe & NH2-sp12-d(T) ${ }_{90}$ \\
NALF Test Line & anti-Digoxin \\
NALF Control Line & biot-TEG-d(A) ${ }_{60}$ \\
\hline DIG = digoxygenin; sp9 = C9 spacer; biot-TEG = biotin-tetra-ethylene glycol; sp18 = C18 spacer; sp12 = \\
C12 spacer
\end{tabular}

EXPAR was also executed in the cartridge, which is initiated by a short trigger oligonucleotide (Trigger X) that can be generated from a targeted site within genomic DNA through the Fingerprinting reaction. ${ }^{58}$ Trigger $\mathrm{X}$ primes and is extended along an oligonucleotide provided in the EXPAR master-mix, called the first stage amplification template. The template consists of two times the complimentary sequence X', separated by nine bases that enable generation of a nicking enzyme recognition site upon polymerase extension. The nicking enzyme, Nt.Bst, recognizes this sequence and reaches four bases to the 3' end of the recognition site to nick the top strand. This nick creates another short oligonucleotide trigger that melts off the amplification template. The 
polymerase elongates the recessed 3'-hydroxyl, created by the departing trigger, and the process repeats. Newly formed triggers then prime other amplification templates, creating true chain (exponential) reactions. Trigger $\mathrm{X}$ is generally 10 to 16 bases long, although the genomic sequence required for trigger generation is much longer (typically $\sim 28 \mathrm{bp}$ ). In two-stage EXPAR, Trigger X is converted to a reporter oligonucleotide (Reporter Y), which can be detected through a colorimetric assay using DNA nanosphere aggregation, ${ }^{59}$ or via NALF, as described herein (Figure 4).
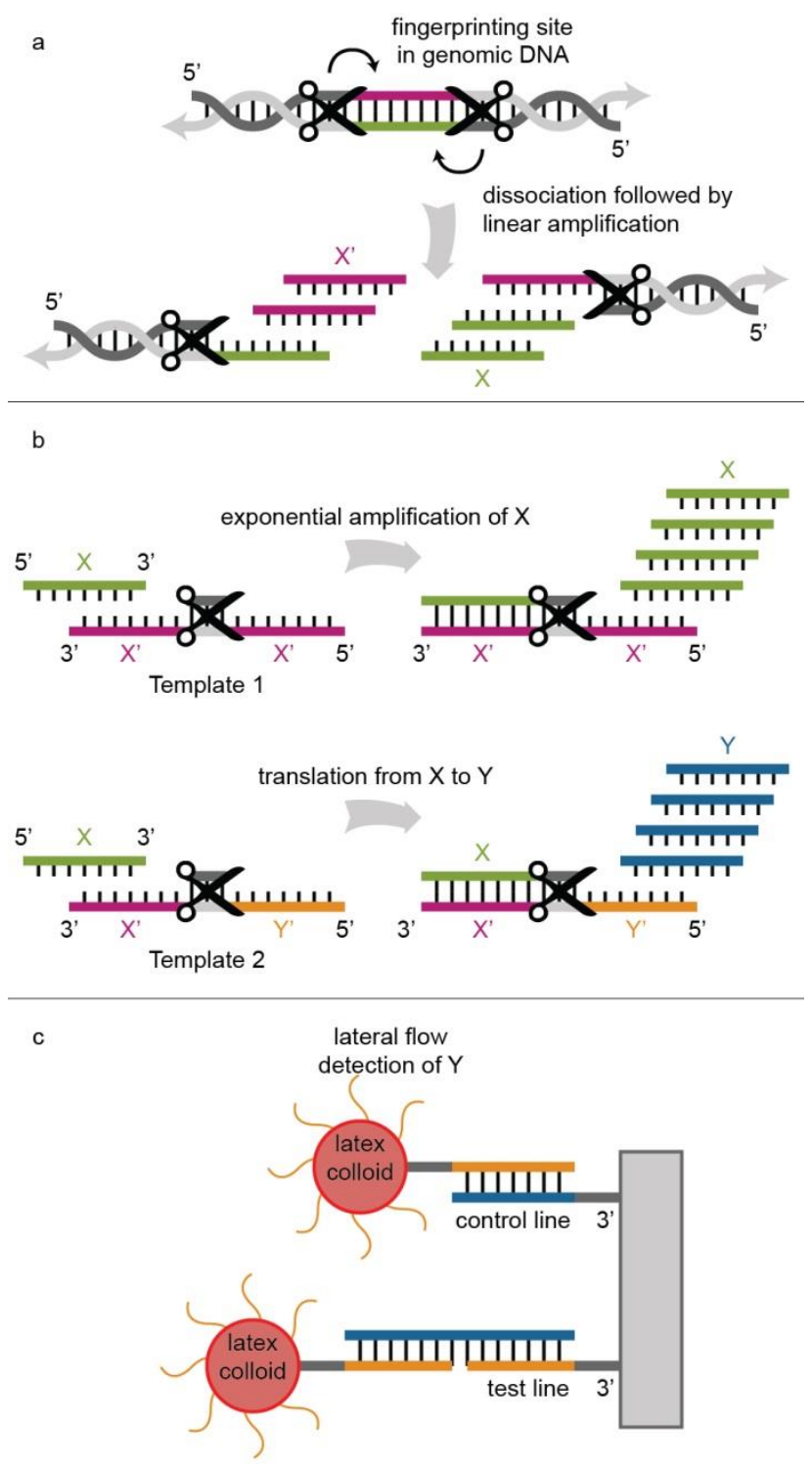
Figure 4: Conceptual depictions of EXPAR reactions and coupling the amplification products with NALF. (a) First-stage EXPAR, where Trigger X primes and is extended along a template provided in the EXPAR master-mix. (b) Second-stage EXPAR illustrating Trigger X converting to a reporter oligonucleotide (Reporter Y). (c) NALF coupled to second-stage EXPAR, where the Reporter Y oligonucleotide binds DNAfunctionalized microspheres and DNA hybridized on the nitrocellulose at the test line, resulting in a visible test line. This binding is dependent on the presence of Reporter Y, so the test line will only be present in positive samples. Additional DNA functionalized on the microspheres will bind DNA hybridized on the nitrocellulose at the control line, resulting in a visible line. The binding at the control line is independent of the presence of Reporter Y, and, therefore, acts as an indicator of proper lateral flow function.

EXPAR was carried out in a reaction mixture containing 0.3 units/ $\mu \mathrm{L} \mathrm{Nt} \mathrm{Bst}$ nicking enzyme (New England Biolabs, Ipswich, MA), 0.03 units/ $\mu$ L Bst polymerase (NEB), 0.24 mM dNTPs (Fermentas, Glen Burnie, Maryland), 1.6 mg/mL bovine serum albumen (New England Biolabs), 3 mM magnesium chloride (Sigma-Aldrich, St. Louis, MO), 1x SYBR Green I (Invitrogen), 20mM Tris $\mathrm{pH} 7.9,15 \mathrm{mM}$ ammonium sulfate, 30mM potassium chloride, $0.005 \%$ Triton $\mathrm{X}-100,1.6 \mathrm{mg} / \mathrm{mL}$ BSA, $25 \mathrm{nM}$ first stage EXPAR template, and $50 \mathrm{nM}$ second stage EXPAR template. Positive reactions contained $6 \times 10^{5}$ copies of M.tb H37Ra genomic DNA, but not in the no trigger control (NTC) negative reactions. Template and trigger sequences are shown in Table 3. Each reaction further contained $10 \mathrm{ng}$ human genomic DNA (Promega, Madison, WI) per reaction as general background. In parallel with experiments performed on the cartridges, 
positive and negative control reactions ( $75 \mu \mathrm{L}$ volume) were amplified in a Bio-Rad (Hercules, CA) MJ Opticon II real-time thermocycler set to a constant temperature of $55^{\circ} \mathrm{C}$, and the fluorescence intensity was monitored in 10 second intervals using excitation at $488 \mathrm{~nm}$ over a period of up to 2 hours to verify reaction kinetics.

NALF Test Strip Fabrication: LAMP-NALF test strips were fabricated in-house, using the probe and control oligonucleotides shown in Table 2, synthesized by Eurofins MWG Operon (Huntsville, AL). Oligo d(T) $)_{90}$ and neutravidin were conjugated respectively to red-dyed carboxylate modified polystyrene microspheres $(0.4 \mu \mathrm{m}$; Thermo Fisher Scientific) using 1-Ethyl-3-[3-dimethylaminopropyl] carbodiimide hydrochloride (EDC) as the cross-linker. Following the conjugation process, the microspheres were resuspended and stored in $50 \mathrm{mM}$ Sodium Borate, $\mathrm{pH} 8.4$, at a final microsphere concentration of $1 \%$. The anti-Digoxin antibody (MyBioSource, San Diego, CA) was diluted to $1 \mathrm{mg} / \mathrm{ml}$ in $10 \mathrm{mM}$ Tris, $\mathrm{pH} 7.4$, plus $5 \%$ ethanol and the biotinylated control line $d(A)_{60}$ oligonucleotide was coupled to neutravidin at room temperature. Both were then dispensed as even and reproducible lines onto a nitrocellulose membrane (Sartorius, Goettingen, Germany) using a lateral flow reagent dispenser (ALFRD 07.711.01, Claremont BioSolutions, Upland, CA). After dispensing the capture antibody and the control line oligonucleotide probe, the nitrocellulose was dried at room temperature for at least 30 minutes, and was subsequently stored in a sealed, desiccated, moisture-barrier pouch.

EXPAR-NALF test strips were also fabricated in-house, using the probe, test, and control oligonucleotides shown in Table 3, obtained from Operon Biotechnologies 
conjugated polystyrene microspheres were then dispensed as a line onto the conjugate pad using the reagent dispenser set to a flow rate of $7 \mu \mathrm{l} / \mathrm{cm}$. The conjugate pad was dried for 5 minutes at $55^{\circ} \mathrm{C}-60{ }^{\circ} \mathrm{C}$, and then stored in a sealed, desiccated, moisture-barrier pouch.

To prepare test strips, we mounted the dispensed nitrocellulose membrane to an adhesive plastic backing ( $\mathrm{G} \&$ L Precision Die Cutting, San Jose, CA). The treated glass fiber conjugate pad and a cellulose fiber absorbent pad (Millipore Corp.) were respectively attached to the bottom (sample) and the top portions of the adhesive backing, each with a 1 - $2 \mathrm{~mm}$ overlap with the nitrocellulose membrane. The resulting sheet was cut into $4 \mathrm{~mm}$ x $50 \mathrm{~mm}$ individual test strips for assembly into the cartridge.

Amplification and Detection in the Cartridge: For amplification and detection in the cartridge, we pre-heated the empty cartridge on the heater for 10 minutes. In LAMP cartridges, LAMP master-mix solutions for the positive and NTC reactions (100 $\mu 1$ each) were then injected into the reaction pouches, and amplified on the heater for 10 minutes. In EXPAR cartridges, EXPAR master-mix solutions for the positive and NTC reactions (75 $\mu 1$ each) were then injected into the reaction pouches, and amplified on the heater for 60 minutes. For all cartridges, electrolytic pumping was initiated after heating to push the amplified master-mixes out of the reaction pouches and into the lateral flow pouches, where the samples migrated along the NALF strips. The results were visually interpreted by reading the test and control lines 10 minutes after pumping was initiated. 


\section{Results and Discussion}

Fluidic Control: This cartridge utilizes electrolytic pumping and therefore does not require external pistons or actuators, as opposed to other clinical diagnostic systems using flexible pouches. ${ }^{21,22,28}$ Similar to previous reports, ${ }^{53}$ we have observed a roughly linear relationship between applied current and flow rate for electrolytic pumping. To ensure that the lateral flow strips perform properly, we determined that amplified master-mix needs to be pumped from the reaction chamber into the lateral flow strip chamber at a flow rate of approximately $100 \mu \mathrm{L} / \mathrm{min}$, which was obtained by applying $50 \mathrm{~mA}$ of current.

One-way valves are necessary to prevent the fluid within the reaction chamber from leaking prematurely into the lateral flow detection chamber. Injecting 75-100 $\mu \mathrm{L}$ liquid into the reaction chamber results in $<2$ PSI fluid pressure, which is less than the cracking pressure of the valves ( 2 PSI) used in the current design. Therefore, fluid is retained in the reaction pouch until electrolytic pumping forces the fluid through the valve and onto the lateral flow strip.

Instrument Design and Thermal Control: Maintaining an appropriate and uniform temperature throughout the reaction pouch during the amplification period is essential for assay performance. With a cartridge inserted into the device, the heater surface requires approximately three minutes to heat from room temperature to $63{ }^{\circ} \mathrm{C}$, and maintains this constant temperature to within $\pm 0.1{ }^{\circ} \mathrm{C}$ for as long as the heater is turned on. Comparable temperature stability is obtained at other temperatures, e.g. $55^{\circ} \mathrm{C}$ required for EXPAR. 
Air trapped between the fluid inside the reaction pouch and heater surface significantly lowers the thermal conductivity across this interface, and can contribute to non-uniform fluid heating within the reaction pouch. This concern can be mitigated by filling the pump pouch with fluid. The slightly inflated pump pouch presses up against the heater surface, which compensates for imperfections on the cartridge bottom surface that prevent the cartridge from sitting completely flat on the heater. Additionally, the fluid in the pump pouch effectively conducts heat to the reaction pouch. We measured the temperature of liquid in the reaction pouch using a thermocouple inserted through the inlet septum. If the pump pouch is empty, then the fluid temperature in the reaction pouch deviates significantly from the temperature measured directly on the heater surface underneath the cartridge (Figure 5a). Filling the pump pouch improves the thermal transfer efficiency between heater surface and cartridge, resulting in temperature equilibration after $\sim 10$ minutes, and a final temperature closer to the desired set-point. Thermal paste applied between the heater surface and pump pouch provides no further improvements. 

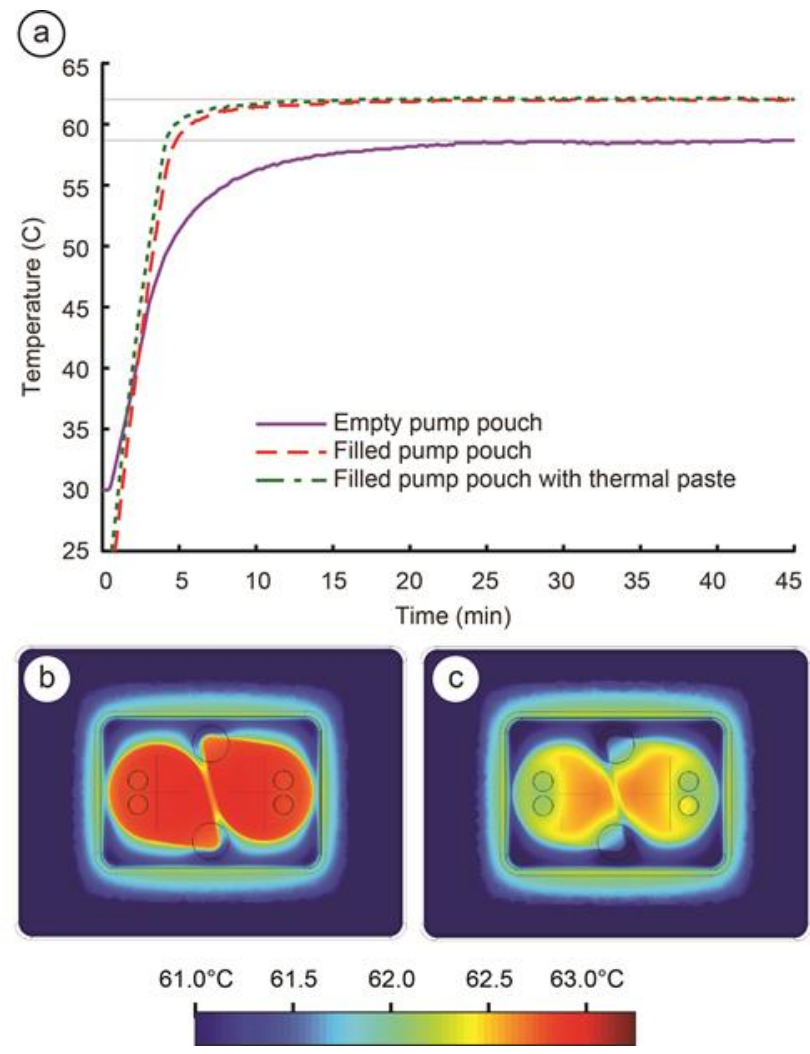

Figure 5: (a) Measured temperature of the fluid inside the reaction pouch of a cartridge attached to the heater as a function of time. Filling the pump pouch with liquid improves the thermal transfer from the heater to the reaction fluid, which reaches a stable temperature of $62 \pm 0.1{ }^{\circ} \mathrm{C}$ within approximately ten minutes. Thermal paste applied between the heater surface and pump pouch provides no further improvements. (b) and (c) Thermal simulations (COMSOL Multiphysics) of the cartridge on top of the heater, showing horizontal cross-sections within the center of the reaction fluid layer of the cartridge. (b) Uniform heating to the desired temperature $\left(63^{\circ} \mathrm{C}\right)$ is observed using a model with ideal thermal contact between cartridge and heater, with no air gap. (c) Introducing a $150 \mu \mathrm{m}$ thick thermally-resistive layer between cartridge and heater leads to a lower temperature and less uniform heating within the reaction pouch. The crosshatches in the middle of the pump pouches indicate the dimensions of the circular 
reaction pouches. An air bubble was intentionally introduced into the outlet port of the reaction chamber to simulate air trapped in the reaction pouches after fluid is inserted.

Using our mathematical model, we found that the temperature within the reaction pouches deviated from $63{ }^{\circ} \mathrm{C}$ by less than $0.5^{\circ} \mathrm{C}$ throughout the reaction chamber if the cartridge makes ideal contact with the heater surface (Figure 5b). To understand the effects of non-ideal thermal contact, we modeled the system with a $150 \mu \mathrm{m}$ thick thermally-resistive air layer between the pump pouches and the heater surface (Figure $5 \mathrm{c}$ ). This resistive layer significantly reduced the heat transfer between the heater and cartridge, decreasing the maximum temperature in the reaction pouches by $1^{\circ} \mathrm{C}$, and leading to more significant temperature variation throughout the reaction pouch.

Isothermal Amplification Methods: We designed out system to work with any isothermal amplification method coupled to lateral flow, to detect genetic material of any disease. For preliminary studies, we have decided to run two amplification methods targeting M.tb genomic DNA: LAMP and EXPAR. These reactions have distinct advantages and disadvantages in terms of integration into the cartridges. For example, in previous work we optimized coupling EXPAR amplification to NALF detection. ${ }^{61}$ Therefore, for EXPAR implementation in the cartridges, we needed to design EXPAR templates for detection of M.tb genomic DNA, and demonstrate target amplification with NALF detection. On the other hand, a LAMP assay was previously clinically-validated in another lab for the detection of $M . t b .{ }^{38}$ Consequently, for LAMP implementation in the cartridges, we needed to develop a method for coupling LAMP to NALF, as described 
below. By using two different amplification methods coupled to lateral flow detection, we are able to demonstrate proof of concept for the cartridge and handheld heater system as a platform technology which could be modified for other applications.

Lateral Flow Detection on Cartridge: The cartridge was designed to run two samples per cartridge. This dual-processing design could increase throughput by allowing for multiple simultaneous tests, or could allow for duplicate testing on one cartridge from the same original sample.

Additionally, the lateral flow strips were positioned in anti-parallel orientation on the top surface of the cartridge. This design was developed as a way to best use the footprint of the cartridge top surface while also utilizing the existing inlet and outlet fluid conduits between the top and bottom cartridge pieces. The lateral flow strips were modified to be shorter $(\sim 4 \mathrm{~cm})$ than standard lateral flow strips $(\sim 5 \mathrm{~cm})$, to facilitate installation on the cartridges. By orienting the strips anti-parallel, we were able to fix the lateral flow strip pouches over outlet fluid conduit, to connect directly with the reaction pouch chamber, but without covering the matching inlet fluid conduit to the other reaction pouch chamber on the opposite end of the cartridge.

Isothermal Amplification Coupled to NALF Detection: To establish proof of principle that isothermal amplification coupled to lateral flow detection can be executed in the cartridge on the heater device, we chose two isothermal amplification reactions that detect M.tb genomic DNA as the target. The first reaction is an established, clinicallyvalidated LAMP assay that has been used for TB diagnosis in low resource settings. ${ }^{38,39}$ 
We have modified this assay to enable coupling with NALF detection (Figure 6).

Coupling LAMP to NALF has been demonstrated for other assays, ${ }^{46}$ but the complicated, large amplicon structure can cause steric hindrance that may result in compromised line intensities and test results.

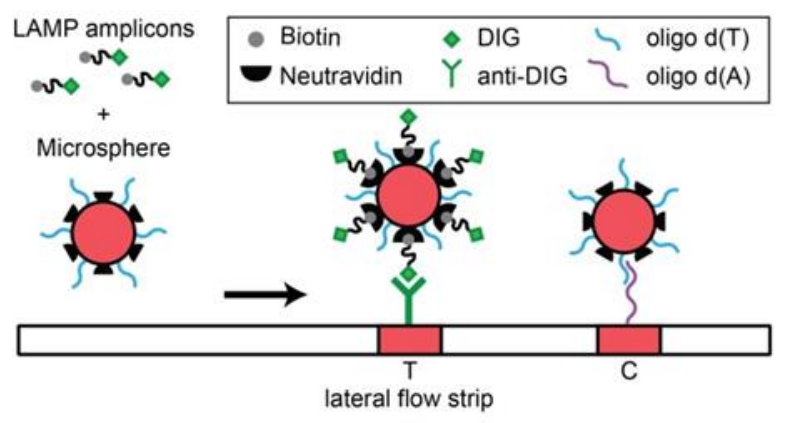

(a)

(b)

(c)

Figure 6: Conceptual depiction of NALF detection coupled to LAMP. (a) Amplified master-mix applied to the conjugate pad enables amplicons to interact with colored polystyrene microspheres functionalized with appropriate capture moieties. (b) After migrating along the nitrocellulose membrane, microspheres carrying amplicons are captured at the test line. (c) At the control line, microspheres are captured irrespective of the presence of amplicon.

Our approach for LAMP amplicon detection via NALF uses a somewhat different reaction scheme than that described for coupling EXPAR to NALF. In positive reactions, the LAMP master-mix contained biotin/DIG-labeled amplicons. Through the biotin moiety, amplicons were captured by the neutravidin-conjugated microspheres. After migrating along the nitrocellulose strip, microspheres carrying LAMP amplicons were captured at the test line $(\mathrm{T})$, based on the interaction between the DIG-labeled portion of 
the LAMP product and the immobilized anti-DIG antibody (Figure 6b). At the control line (C), the oligo-d(A) immobilized on the nitrocellulose membrane hybridized to the oligo-d(T) conjugated to the microspheres (Figure 6c). This control line confirms proper performance of the lateral flow strip, and should appear in positive and negative reactions as hybridization is independent of the presence of LAMP amplicons. In contrast, the test line should only appear in positive reactions. In addition, many NALF reactions require an extra liquid running buffer, which introduces another reagent and complicates the device design. In our LAMP-NALF scheme, amplified master-mix can be applied directly to the conjugate pad of the NALF strip, where it reconstituted a dry reagent mixture containing colored microspheres. No other liquid reagents are required other than the amplified master-mix.

The second assay used herein is EXPAR, which amplifies short trigger oligonucleotides at $55{ }^{\circ} \mathrm{C}$ using polymerase and nicking enzyme activities. ${ }^{58}$ In prior work, we have demonstrated that the trigger oligonucleotide amplified in EXPAR can be generated from a targeted genomic DNA sequence that contains adjacent nicking enzyme recognition sites through the Fingerprinting reaction, ${ }^{61}$ and have implemented EXPAR in a two-stage reaction format to generate a reporter oligonucleotide that can be detected via a nanosphere based colorimetric readout. ${ }^{59}$ We detected M.tb genomic DNA through a Fingerprinting two-stage EXPAR assay with NALF-based readout. Two-stage EXPAR generates large amounts of single-stranded reporter oligonucleotide $\mathrm{Y}$ that can form a sandwich complex with complementary probe oligonucleotides conjugated to microspheres and to the test line of a NALF strip (Figure 4c). Therefore, the test line becomes visible in positive reactions. The probe oligonucleotide immobilized at the 
NALF control line hybridizes directly to the probe sequence conjugated to the microspheres. Therefore, the control line appears in positive and negative reactions. Again, we developed an EXPAR-NALF strip with a dry reagents mixture in the conjugate pad, to which amplified master-mix can be applied directly without the need for additional liquid reagents.

Amplification and Detection in the Cartridges: We tested the performance of both isothermal assays coupled to NALF detection in the cartridge prototype. For these experiments, the heater set-point was adjusted for the desired reaction temperature $\left(63{ }^{\circ} \mathrm{C}\right.$ for LAMP and $55^{\circ} \mathrm{C}$ for EXPAR), and a cartridge with empty reaction chambers was allowed to pre-heat on the heater for ten minutes. We then injected master-mix through the inlet septum into the reaction chambers, with one positive and one negative reaction per cartridge. We allowed the reaction to incubate on the heater for 10 minutes (LAMP) or 60 minutes (EXPAR), then applied current to the electrolytic pumps, and allowed 10 minutes for NALF detection. The final readouts for LAMP-NALF (Figure 7a) and EXPAR-NALF (Figure 7c) consisted of the expected two lines $(\mathrm{T}=$ test and $\mathrm{C}=\mathrm{control}$ ) for the positive and a single line (control) for the negative reactions. For LAMP we also determined that master-mix amplified in the cartridge produces the same characteristic pattern of high molecular weight amplicons as control reactions performed under standard conditions (Figure 7b). 

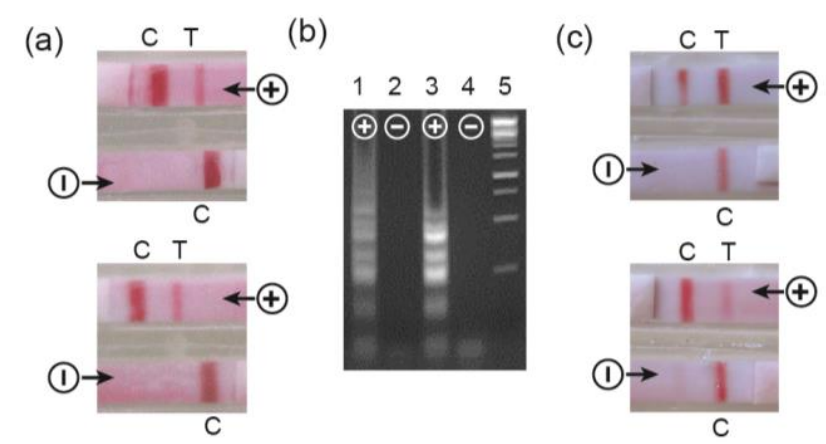

Figure 7: Amplification results. (a) LAMP based detection of M.tb genomic DNA performed in the cartridge on the instrument: NALF strips of two representative cartridges, after 10 minutes of isothermal amplification, followed by $10 \mathrm{~min}$ for lateral flow detection. (b) LAMP master-mix amplified in the cartridge on the heater, analyzed via gel electrophoresis (Lanes 1 and 2), compared to amplification performed in reaction tubes on a standard heat block (Lanes 3 and 4). Lane 5: DNA molecular weight markers. For (a) and (b), positive (+) reactions show LAMP product starting from 3000 copies of M.tb DNA, and negative (-) reactions show no product since no $M . t b$ DNA was added to the reaction. (c) EXPAR based detection of M.tb genomic DNA performed in the cartridge on the instrument: NALF strips of two representative cartridges, after 60 minutes of isothermal amplification, followed by $10 \mathrm{~min}$ for lateral flow detection. Positive (+) reactions contained $6 \times 10^{5}$ copies M.tb DNA, and negative (-) reactions contained no M.tb DNA.

For LAMP, positive reactions contained 3000 copies of $M . t b$ genomic DNA, while for EXPAR, positive reactions contained $6 \times 10^{5}$ copies of $M . t b$ genomic DNA. Relatively high concentrations of target genomic DNA were chosen as a means of establishing proof of principle for the cartridge prototype. The goal of the study reported 
herein was not to test the limit of detection for the assays. In both cases, negative reactions contained no M.tb genomic DNA, but all reactions included $10 \mathrm{ng}$ human genomic DNA, which is present as background in clinical samples. The assays are not cross-reactive with human genomic DNA. LAMP produced results after 10 minutes of isothermal amplification, likely due to the relatively high starting copy number. ${ }^{38}$ The EXPAR assay, which still needs to be optimized, required longer amplification under the current reaction conditions.

\section{Conclusions}

Using the system and assay reported herein, we have demonstrated detection of purified M.tb genomic DNA through isothermal LAMP and EXPAR reactions coupled with NALF in a fully-enclosed combined format. Through this early prototype, we obtained suitable thermal control, and have established proof of principle for the novel fluid handling approach using two-layer pouches combined with one-way valves and electrolytic pumping. This system is capable of executing different isothermal amplification reactions coupled with NALF detection, such as the LAMP and EXPAR assays described herein.

This system was developed as a step towards the goal of enabling point-of-care infectious disease diagnosis in low-resource settings. We are refining the design of this amplification and detection subunit ${ }^{62}$ (Chapter 4) to incorporate miniaturized and integrated electrolytic pumps and novel passive valves ${ }^{63}$ (Chapter 3), and to enable automated pumping, heating, and timing. 


\section{CHAPTER 3: NOVEL MINIATURE PASSIVE VALVE}

\section{Introduction}

Valves are an essential component for the control and manipulation of fluids. Many passive and active valve designs exist, each with their own advantages and limitations. ${ }^{64-}$ ${ }^{68}$ The small passive valves presented in this paper offer a combination of attributes not readily met by commercially available stand-alone passive valves, such as ball-andspring, duckbill, and umbrella valves. These attributes include small dead volume, selectable non-zero cracking pressure, and the ability to be mass manufactured at low cost. Valves used in medical diagnostics and devices also have to be compatible with certain biomaterials, such as enzymes and other reagents used in clinical diagnostic applications. ${ }^{68-70}$ Our disposable cartridges for point-of-care nucleic acid testing require passive check valves with the attributes mentioned above. ${ }^{62}$ None of the currently available commercial options were able to meet all our desired criteria. For example, the Lee Company (Westbrook, CT) produces a ball-and-spring valve that meets all the physical requirements but is cost prohibitive. The remaining valve options all had recommended installation configurations that created a dead volume too large for a typical diagnostic assay, among other challenges.

Specialized valves made using microfabrication techniques, ${ }^{67,71-76}$ such as photolithography, can be very small and often have negligible dead volumes. However, these techniques require the valve to be, at least in part, fabricated in the same way and at the same time as the system with which it is integrated. This limits the design options greatly. Furthermore, linking macro and micro volumes in microfabricated designs is a significant challenge, one that is essential for diagnostic systems that require large initial 
sample input volumes and much smaller volumes during final analysis. The valve design presented herein can be made using commercially available sub-components, is low cost and easy to assemble. The valve is manufactured independently from the system into which it is inserted. Its dead volume, although larger than lithography-based valves, is significantly lower than that of commercially available stand-alone solutions with competitive manufacturing costs.

\section{Experimental}

Design and Operating Concept: The body of the valve consists of a hollow cylindrical core, capped at one end, with a side port (Figure 8). An elastic cylindrical sleeve is stretched over the core covering the side port (Figure 8b). When sufficient positive pressure is applied to the interior of the core, the sleeve expands and allows flow through the side port between the exterior of the core and interior of the sleeve. The radially oriented pressure out the side port in conjunction with the friction between the sleeve and core prevents the sleeve from slipping off. Once the pressure is reduced, the sleeve returns to its resting diameter on the core, closing the port and preventing backflow. The valve is easily integrated into a system by fitting the open end of the core into the fluid path. It can be used in an open configuration, letting fluid flow out into a reservoir, or it can be encapsulated to create an in-line check valve (Figure 8c). Similarly functioning

valve designs have been proposed. ${ }^{77-79}$ However, the non-cylindrical geometries proposed in these patents are likely to increase their manufacturing challenges, which is, perhaps, the reason these valves are not commercially available. 
a

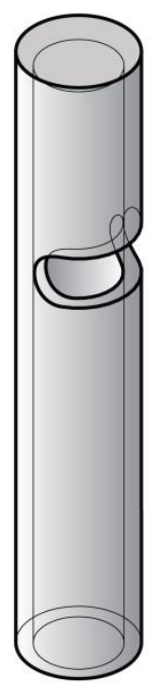

b

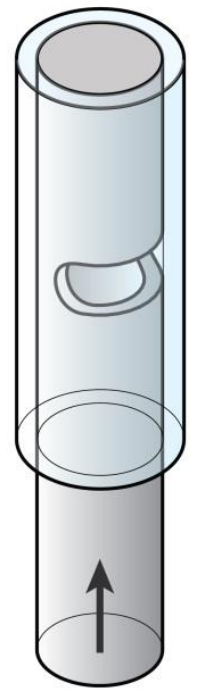

C

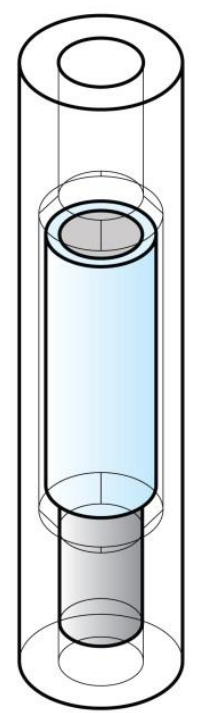

Figure 8: Valve design components: (a) inner rigid core with one end capped and one side port; (b) rigid core with elastomeric sleeve covering side port; (c) encapsulated valve for in-line use.

The cracking pressure of the herein described valves can be tuned to a desired value by adjusting easily controllable valve dimensions. We developed a mathematical model for an idealized case, using elasticity theory of thick-walled cylinders, which predicts that the cracking pressure of the valve is dependent solely on the modulus of the sleeve material, the outer diameter of the core, and the inner diameter and wall thickness of the sleeve. Although this model is based on an idealized case, we expect that we can create valves with different and tunable cracking pressures by varying the above mentioned core and/or sleeve geometries.

In reality other factors likely contribute to the valve performance, such as small adhesive forces and/or friction between the core outer surface and the inner surface of the sleeve, and axial deformation of the sleeve. Such additional forces likely contribute to the two distinct observable pressures associated with the opening of the valve. First, there is 
an initial pressure spike indicating the true cracking pressure, where the pressure inside the valve core is large enough to stretch the sleeve open, releasing the dead-air trapped inside the valve core. We refer to this initial spike as the valve cracking pressure. After this initial pressure spike, there is a lower pressure associated with maintaining the valve in the open position. We refer to this pressure as the sustained open pressure.

Valve Fabrication: According to our model, the valve cracking pressure depends predominantly on the diameters of the core and sleeve, which are made from rigid and elastic tubing readily available with precise diameters at low costs. The tolerances on other features, such as the geometry of the side port, length of the sleeve, or position of the sleeve, depend mainly on additional considerations such as valve seating and method of insertion into the final device. The tolerances for these other parameters are larger than the needed tolerances for core OD, sleeve ID, and sleeve wall thickness, which simplifies valve manufacturing and assembly, since these other features of the valve are substantially more expensive to manufacture to the same precision.

Two different embodiments of the valve were assembled and tested (Figure 9). The first valve consisted of a core that was fabricated from stainless steel hypodermic tubing (Small Parts, Inc), sliced into short sections using a cutting wheel on a rotary tool. We then cut a notch on the side into each core tubing segment to create the side port, and sealed one end of each segment with UV curing glue (KOA 300, Kemxert Co.) or with melted polycarbonate. The valve sleeve was made from silicone tubing (VWR International, LLC), which was pushed over the core, covering the side port. A puff of air was injected into the valve to inflate the sleeve tubing above the cracking pressure. This 
relieved any axial deformation that may have occurred while placing the sleeve on the core. Excess silicone was cut away with scissors. We fabricated two sets of test valves using this method, with alternate dimensions for the core and sleeve (Table 4). The second valve embodiment consisted of a core fabricated from PEEK tubing (Zeus Inc.), sliced into short segments using a razor blade. We then cut a notch into the side, and sealed one end of the core by melting the PEEK material. The valve sleeve was made by casting silicone (R 1328, Silpak Inc.) into short cylinders with varying inner diameters and wall thicknesses. These sleeves were then pushed over the PEEK core, covering the side port. As for the previous design, a puff of air was injected into the valve to relieve any axial deformation of the sleeve. We fabricated several sets of test valves with constant core dimensions and sleeve wall thickness, but with varying sleeve inner diameters (Table 4, Figure 9).

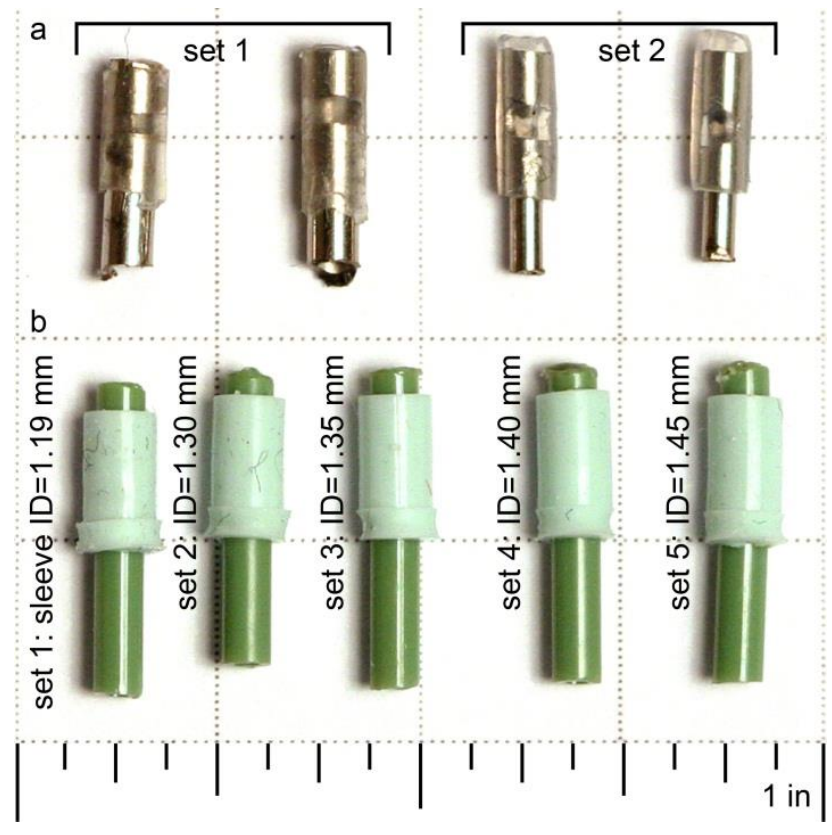


Figure 9: Different embodiments of the sleeve valve. (a) Stainless steel core tubing with silicone tubing sleeve. (b) PEEK core tubing with cast silicone sleeves, with varying sleeve inner diameters, as indicated in the figure.

Table 4: Key dimensions and physical parameters for three valve sets.

\begin{tabular}{cccc}
\hline & Stainless Steel Valve Set 1 & Stainless Steel Valve Set 2 & PEEK Valves \\
\hline Core Outer Diameter & $0.065 \pm 0.0005^{\prime \prime}$ & $0.042 \pm 0.0005^{\prime \prime}$ & $0.061 \pm 0.0003^{\prime \prime}$ \\
& $(1.65 \pm 0.01 \mathrm{~mm})$ & $(1.07 \pm 0.01 \mathrm{~mm})$ & $(1.56 \pm 0.007 \mathrm{~mm})^{\mathrm{c}}$ \\
Core Inner Diameter & $0.047 \pm 0.0005^{\prime \prime}$ & $0.027 \pm 0.0005^{\prime \prime}$ & $0.03 \pm 0.002^{\prime \prime}$ \\
& $(1.19 \pm 0.01 \mathrm{~mm})$ & $(0.69 \pm 0.01 \mathrm{~mm})$ & $(0.76 \pm 0.05 \mathrm{~mm})$ \\
Sleeve Inner Diameter ${ }^{\text {a }}$ & $0.058^{\prime \prime}(1.47 \mathrm{~mm})$ & $0.030^{\prime \prime}(0.76 \mathrm{~mm})$ & $0.047-0.057^{\prime \prime}$ \\
Sleeve Wall Thickness ${ }^{\mathrm{a}}$ & $0.009^{\prime \prime}(0.229 \mathrm{~mm})$ & $0.018^{\prime \prime}(0.46 \mathrm{~mm})$ & $0.017^{\prime \prime}(0.43 \mathrm{~mm})$ \\
$\begin{array}{c}\text { Sleeve axial modulus of } \\
\text { elasticity }\end{array}$ & $482 \pm 37$ PSI & $229 \pm 19 \mathrm{PSI}$ & $79.0 \pm 0.1 \mathrm{PSI}$ \\
\hline
\end{tabular}

${ }^{a}$ Sleeve inner diameter and wall thickness when sleeve is relaxed. ${ }^{b}$ Determined experimentally by performing a tensile test (Instron ${ }^{\circledR}$ ) with 10 replicates for each sleeve material. ${ }^{\mathrm{c}}$ Measured for actual PEEK tubing batch used in valve construction. ${ }^{d}$ Five valve sets were fabricated with different sleeve inner diameters, see Figure 9.

Valve Testing: Each valve tested was connected to a syringe pump and a fluid filled pressure sensor (Omega Engineering, Inc) via a T-junction. At the outlet, the valve was open to atmosphere such that the gauge pressure measured by the sensor represented the pressure drop across the valve, which was recorded over time.

\section{Results and Discussion}

Cracking and Sustained Open Pressures: We determined the cracking and sustained open pressures for the stainless steel and PEEK core valves (Table 5). With the syringe pump set to a fixed flow rate, the pressure upstream of the valves increased until it reached the 
cracking pressure. Thereafter, the pressure held steady at the sustained open pressure (Figure 10a i and ii, respectively).

Table 5: Cracking and sustained open pressure for different valve sets

\begin{tabular}{cccc}
\hline Valve Type $^{\text {a }}$ & $\begin{array}{c}\text { Flow rate } \\
{[\mu \mathrm{L} / \mathrm{min}]}\end{array}$ & $\begin{array}{c}\text { Cracking Pressure } \\
{[\mathrm{PSI}]}\end{array}$ & $\begin{array}{c}\text { Sustained Open } \\
\text { Pressure [PSI] }\end{array}$ \\
\hline Steel set 1 & 500 & $6.00 \pm 1.38$ & $5.39 \pm 0.71$ \\
Steel set 1 & 200 & $4.93 \pm 1.09$ & $4.74 \pm 0.75$ \\
Steel set 2 & 500 & $19.89 \pm 1.73$ & $19.29 \pm 1.77$ \\
PEEK set 1 & 500 & $7.41 \pm 0.57$ & $4.82 \pm 0.30$ \\
PEEK set 2 & 500 & $6.64 \pm 0.43$ & $3.76 \pm 0.22$ \\
PEEK set 3 & 500 & $6.22 \pm 0.63$ & $3.41 \pm 0.20$ \\
PEEK set 4 & 500 & $5.31 \pm 0.58$ & $2.94 \pm 0.19$ \\
PEEK set 5 & 500 & $5.24 \pm 0.32$ & $2.22 \pm 0.80$ \\
PEEK set 6 & 500 & $7.81 \pm 0.53$ & $6.23 \pm 0.50$ \\
PEEK set $6^{\mathrm{b}}$ & 200 & $7.83 \pm 0.52$ & $6.08 \pm 0.71$ \\
PEEK set $6^{\mathrm{b}}$ & 100 & $7.00 \pm 0.57$ & $5.34 \pm 0.83$ \\
\hline
\end{tabular}

${ }^{\mathrm{a}}$ Five to ten identically manufactured valves tested per data set. ${ }^{\mathrm{b}}$ PEEK set 6 manufactured using sleeves with inner diameter nominally identical to PEEK set 1, but manufactured using a different mold with slight differences in other parameters. 


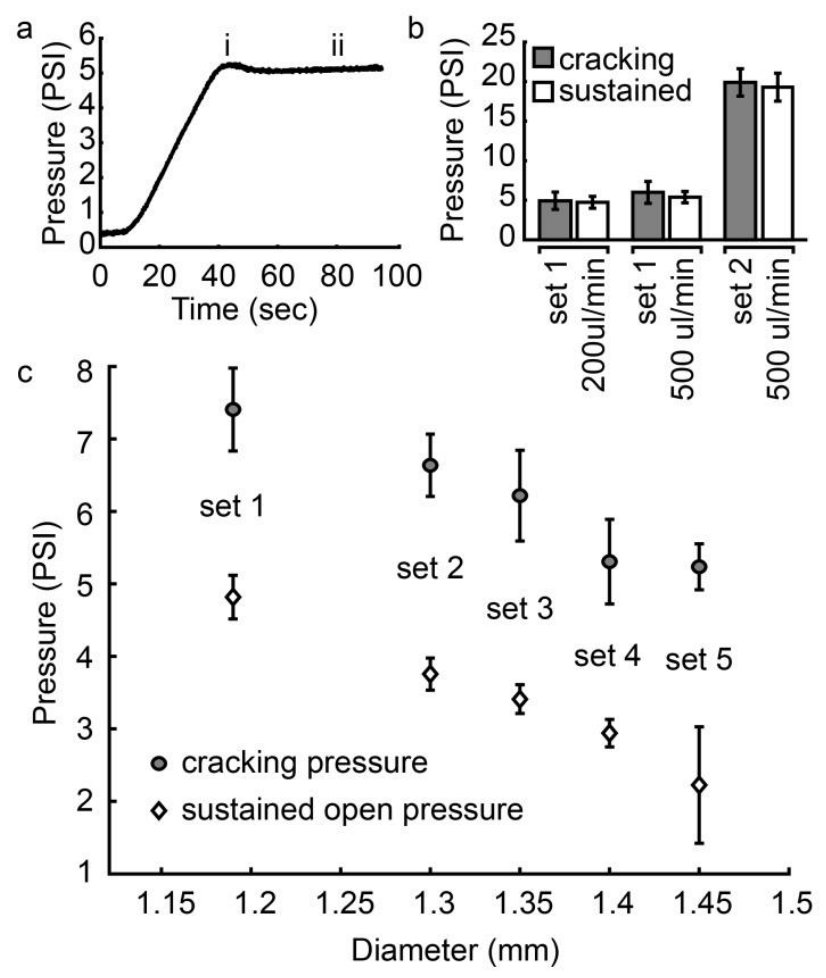

Figure 10: Cracking and sustained open pressure for different valve embodiments. (a) Pressure as a function of time for an example steel valve, indicating (i) cracking pressure and (ii) sustained open pressure; (b) Cracking pressure for the two stainless steel valve sets; (c) Cracking pressure for PEEK valve sets 1-5, as a function of silicone sleeve inner diameter.

All valves tested provided distinct and reproducible cracking and sustained open pressures (Table 5, Figure 10), with pressure values ranging from 2 to 20 PSI, depending on the valve type. This ability to tune the cracking and sustained open pressures enables ready fabrication of valves suitable for different applications. No back flow leakage was encountered prior to exceeding the measurement capabilities of the pressure sensor (30 PSI). Furthermore, the observed trends in cracking pressure agree qualitatively with the 
trends predicted by the model. Steel valve set 2 has significantly larger cracking and sustained open pressure values compared to steel valve set 1 , as expected based on the larger difference between the core outer and sleeve inner diameters, and the larger sleeve wall thickness for set 2. For the PEEK core valves, as predicted by the model, the cracking and sustained open pressure decreased as the inner diameter of the sleeve increased (Figure 10c). Furthermore, upon decreasing the flow rate from 500 to 200, and $100 \mu \mathrm{L} / \mathrm{min}$, the cracking and sustained open pressures for representative steel and PEEK valve sets decreased slightly, by up to 1 PSI (Table 5).

The cracking versus sustained open pressure was not significantly different for the steel valves ( $\mathrm{p}$ value $\geq 0.499$ ), but for the PEEK valves, the cracking pressure was $2.0 \pm$ 0.8 PSI larger than the sustained open pressure ( $\mathrm{p}$ value $\leq 0.00012$ ). We hypothesize that the difference between cracking and sustained open pressure depends primarily on the materials used for the sleeve and core, which dictate the adhesion and friction between the sleeve and core. The cast silicone sleeves appear to have much stronger adhesive interactions with different surfaces, compared to the sleeves obtained from silicone tubing. If having a cracking pressure spike is undesirable for a particular application, then this can be remedied by choosing different sleeve and / or core materials.

Dead Volume: The dead volume within the valve depends on the internal geometry and length of the core. The stainless steel core valves have a dead volume of $15 \mu \mathrm{L}$ for set 1 , and $3 \mu \mathrm{L}$ for set 2 . The dead volume of the PEEK core valves was $4 \mu \mathrm{L}$. The volume can be reduced without changing the cracking pressure or sleeve material by increasing the wall thickness of the core, thereby reducing the internal diameter and dead volume. 
However, reducing the internal diameter of the core increases the overall flow resistance. Therefore, the most suitable core ID has to be selected based on the requirements of a given application.

Compatibility with Nucleic Acid Amplification: We have incorporated the PEEK core valves into our disposable cartridge to execute isothermal nucleic acid amplification coupled to lateral flow detection. ${ }^{62}$ For fluidic systems that execute nucleic acid amplification, components that come in contact with sample or master-mix fluids should be free of the target DNA or RNA, or products of target amplification, to prevent false positive amplification. For our prototype development studies, we found that DNA contamination can be eliminated by subjecting cartridge components to a dry-autoclave cycle for 50 minutes at $120{ }^{\circ} \mathrm{C}$. We dry-autoclaved 8 fully-assembled PEEK core valves, and tested their valve cracking and sustained opening pressures before and after autoclaving (Table 6). The valve cracking pressure decreases slightly after autoclaving, but the difference was not found to be statistically significant $(\mathrm{p} \geq 0.13)$.

Table 6: Cracking pressure for PEEK valves before and after autoclaving

\begin{tabular}{cccc}
\hline Valve Type & Autoclaved & $\begin{array}{c}\text { Flow rate } \\
{[\mu \mathrm{L} / \mathrm{min}]}\end{array}$ & $\begin{array}{c}\text { Cracking Pressure } \\
{[\mathrm{PSI}]}\end{array}$ \\
\hline PEEK set 1 & No & 200 & $6.26 \pm 0.83$ \\
PEEK set 1 & Yes & 200 & $5.56 \pm 0.36$ \\
PEEK set 4 & No & 200 & $4.15 \pm 0.45$ \\
PEEK set 4 & Yes & 200 & $3.69 \pm 0.09$ \\
\hline
\end{tabular}

Four valves of each type were tested at a flow rate of $200 \mu \mathrm{L} / \mathrm{min}$, either in its original form or after dry autoclaving for 50 minutes at $121{ }^{\circ} \mathrm{C}$ prior to the experiment. The valve cracking pressure decreases slightly after autoclaving, but the difference was not found to be statistically significant $(p \geq 0.13)$.

Furthermore, these valves come in contact with the master-mix prior to isothermal DNA amplification in our system. Therefore, we needed to verify that the valve materials 
do not inhibit the DNA polymerase mediated amplification in our isothermal Loop Mediated Amplification (LAMP) reactions. ${ }^{38}$ For these experiments, we incubated a small piece of each valve material, either autoclaved or in its original form, in LAMP reaction buffer for 60 minutes at $63{ }^{\circ} \mathrm{C}$, with agitation by rocking at a speed of $750 \mathrm{RPM}$. We then used this buffer solution in setting up LAMP reactions that were subsequently amplified in the presence and absence of the targeted genomic DNA by incubation at $63{ }^{\circ} \mathrm{C}$, with real time fluorescence monitoring using an Opticon2 real-time PCR instrument (Bio-rad, Hercules, CA). None of the valve materials inhibited the LAMP reactions (Figure 11), and autoclaving did not affect the amplification results. We further demonstrated LAMP based DNA amplification in our cartridge configuration including the valves, ${ }^{62}$ and the valves functioned in this system as expected (Figure 11).

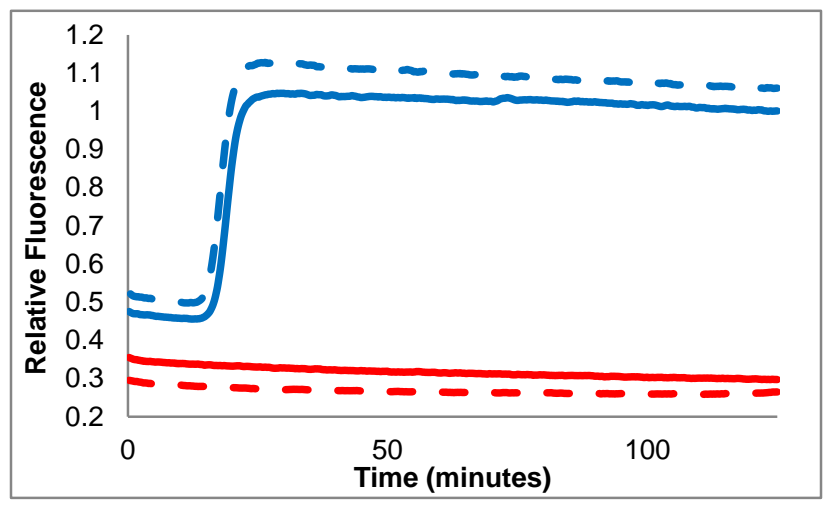

Figure 11: Nucleic acid amplification reactions with (solid lines) and without (dashed lines) autoclaved valve materials incubated in the reaction buffer prior to running the amplification reaction. The valve materials do not inhibit the reaction. A small piece of each valve material, either autoclaved or in its original form, was incubated in LAMP reaction buffer for 60 minutes at $63{ }^{\circ} \mathrm{C}$, with agitation. We then used this buffer solution 
in setting up LAMP reactions that were subsequently amplified in the presence and absence of the targeted genomic DNA by incubation at $63{ }^{\circ} \mathrm{C}$, with real time fluorescence monitoring using an Opticon2 real-time PCR instrument (Bio-rad, Hercules, CA). Blue: LAMP positive reactions containing 3000 copies of target genomic DNA per reaction. Red: LAMP negative control reactions, which did not contain target DNA.

\section{Conclusions}

We demonstrated a stand-alone, miniature check valve design with selectable and reproducible cracking and sustained open pressures. The axial flow alignment of the valve lends itself to be press-fit, or similarly inserted into a flow path. The valves can be fabricated in a simple and reproducible manner from readily available, low-cost materials. The current prototype production process involves manual cutting, sealing, and notching of the rigid core, which alternatively can be accomplished through industry-standard deep draw production methods. Likewise, we used casting to generate elastomeric sleeves of varying inner diameters, but for mass production, the sleeves can be more readily obtained in large volume by injection molding, or by cutting silicone tubing of the appropriate inner diameter and wall thickness into shorter pieces. Therefore, the production of these valves can be scaled up to large-volumes using traditional manufacturing techniques. The valves can be manufactured from non-reactive materials that are compatible with challenging applications such as automation of biological assays. $^{62}$ 


\section{CHAPTER 4: REFINED PROTOTYPE FOR AMPLIFICATION AND DETECTION SUBUNIT}

\section{Introduction}

The work presented herein builds upon an earlier prototype of the amplification and

detection unit ${ }^{80}$ (Chapter 2), comprised of a disposable cartridge containing flexible pouches, passive valves ${ }^{63}$ (Chapter 3 ), and electrolysis-driven pumps, in conjunction with a small heater. Using this refined subunit, we have demonstrated successful execution of an established and clinically-validated isothermal LAMP reaction targeting Mycobacterium tuberculosis (M.tb) DNA, ${ }^{38}$ which we have coupled to NALF detection.

\section{Experimental}

The refined disposable cartridge can execute two isothermal nucleic acid amplification reactions coupled to lateral flow detection in parallel. This enables identification of multiple targets, or amplification of the target $(M . t b)$ in one reaction and an unrelated amplification control in another reaction, which is useful for reactions that cannot be readily multiplexed, such as LAMP.

The operating principle is illustrated in Figure 12a. A sample of purified genomic DNA is injected through a septum into the sample input port. The sample is directed into a chamber containing liquid or lyophilized master-mix reagents using a syringe pump to simulate an upstream integrated electrolytic pump (ePump). Next, the master-mix is pumped through a channel with a hydrophobic barrier that vents the dead air that originally filled the fluid channels, and into the reaction insert. This reaction insert consists of two reaction pouches with inlet and outlet ports containing one-way passive check valves. Each reaction pouch is enclosed by a pump pouch connected to an ePump. 
The reaction insert is located above a heating plate on the instrument, which facilitates isothermal amplification at a set temperature for a pre-determined length of time. Once amplification is complete, the ePump is turned on. Water electrolysis generates gas that pressurizes the fluid in the pump pouch, which then compresses the reaction pouch, forcing the amplified master-mix to exit through the outlet valve and into the lateral flow detection chamber located on the top of the cartridge. The amplified master-mix migrates up the lateral flow strip, generating a positive result if the test and control lines appear visible, or a negative result if only the control line appears visible. The lateral flow detection chamber is vented into the waste chamber through another hydrophobic barrier to reduce pressure build-up as the amplified master-mix is pumped into the chamber. Overall, amplification and detection are executed in an automated fashion in a fullyenclosed cartridge, to prevent amplicon carry-over. 


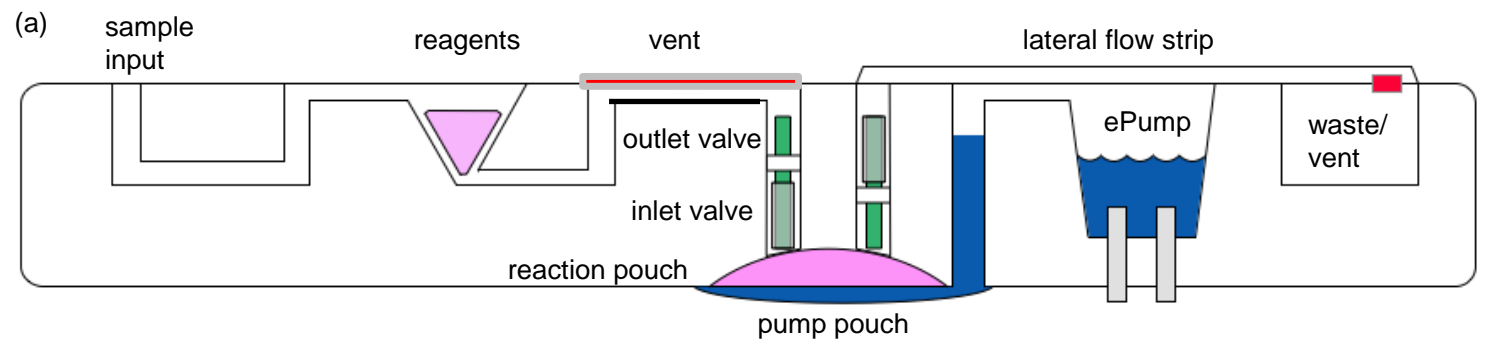

(b)

(c)
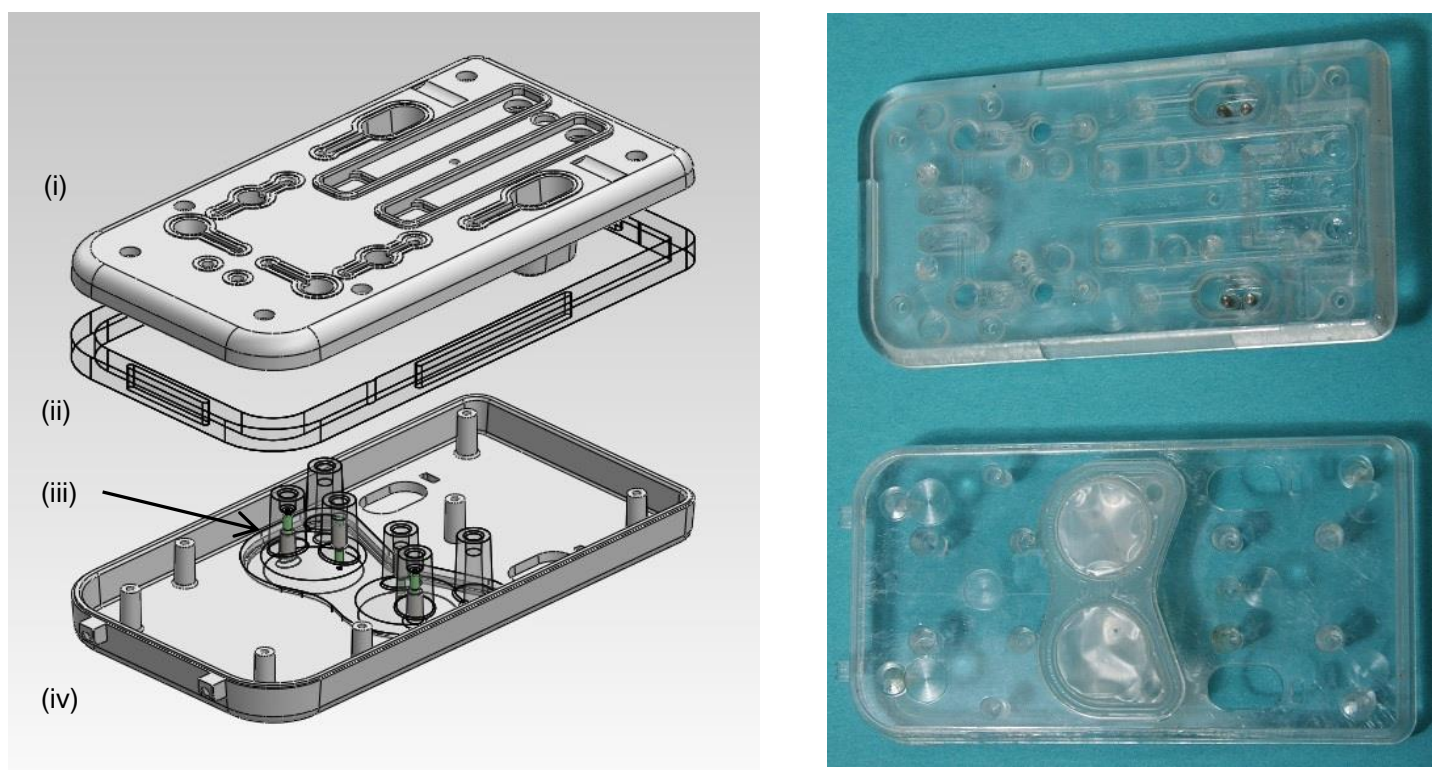

Figure 12: (a) Design concept of the fluidic system of the cartridge. (b) CAD design of the cartridge components including: (i) cartridge core, (ii) spacer ring, (iii) reaction insert, and (iv) bottom casing. (c) Injection molded pieces of the cartridge components.

The cartridge (Figure 12b) consists of a polycarbonate core piece, that incorporates two integrated ePumps, fluidic channels, two chambers that hold master-mix reagents, and two lateral flow detection chambers. The fluidic channels and lateral flow detection chambers are closed via polycarbonate film heat sealed to the top and bottom of the core piece. The cartridge also features a polypropylene insert containing reaction and pump pouches formed via heat sealing polypropylene film onto the rigid polypropylene insert, with check valves press fit into the inlet and outlet ports of the reaction pouch. 
Dead air is removed from the reaction pouches during assembly, and the pump pouches are filled with silicone oil that facilitates hydraulic pumping through a connection with the ePumps, and enhances heat transfer between the heater surface of the instrument and the enclosed reaction chamber. The assembled reaction insert is press fit onto the polycarbonate core, which is then attached to another polycarbonate bottom casing. To protect the insert-molded electrodes from mechanical shock, the electrode pins are recessed within the cartridge by the addition of an acrylic spacer ring that runs along the entire seam between the polycarbonate core and bottom casing pieces. The bottom casing contains access windows to the reaction pouch insert and ePump electrodes, and presses the reaction insert against the core, providing appropriate fluidic seals at the connection points. During assembly, the internal volume of the cartridge is filled with silicone foam to reduce the heat lost from the reaction insert during isothermal amplification.

To demonstrate feasibility of scalable production of the proposed cartridge design, the polycarbonate cartridge core and bottom casing, as well as the polypropylene reaction insert components, were injection molded (Figure 12c). Currently, a maximum of 24 polycarbonate or 100 polypropylene components can be produced in one workday. By improving the mold design and moving the process to an industrial machine with steel molds, we estimate that $\geq 1000$ polycarbonate components and a much larger number of polypropylene inserts could be produced per day on each dedicated machine.

Handheld Instrument: Complementing the refined disposable cartridge, we also refined the prototype instrument to facilitate heating, pumping, and timing. The instrument secures the cartridge in a recessed slot on its top surface. Datum location and spring clip 
mechanisms ensure proper mating of the electrical contacts and the heating surface of the instrument to the ePump connectors and the reaction insert on the cartridge (Figure 13). The on-board microprocessor can be readily programmed to obtain the required assay timing, heater temperature, and current provided to the ePumps, which means that different types of isothermal amplification assays can be readily accommodated. This refined prototype system operates from a plug-in DC power source, but the low-power electronics can be readily operated from an integrated battery pack. The instrument contains three LEDs, of which one indicates appropriate heater temperature and two indicate proper ePump connections, plus a start button to trigger assay execution.

(a)

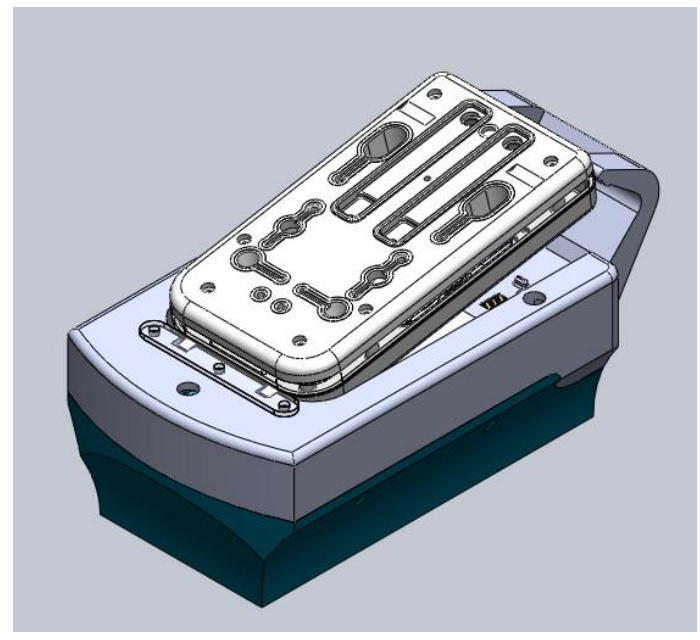

(b)

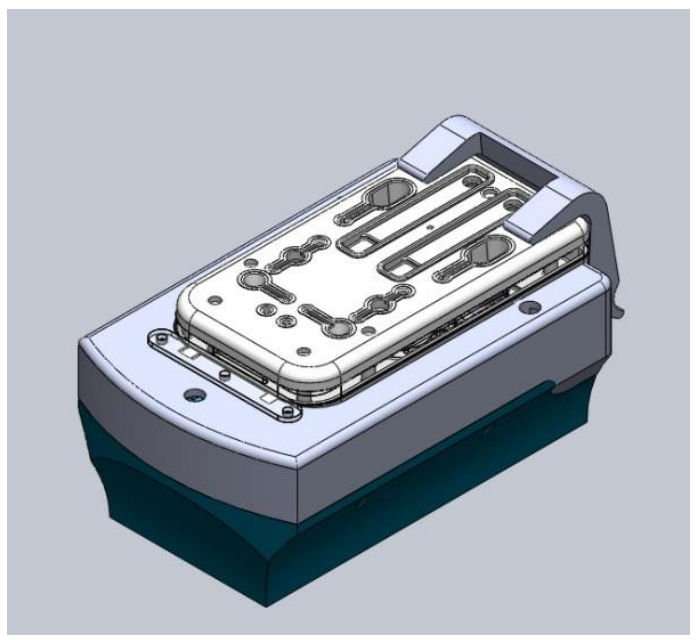

Figure 13: Steps of cartridge insertion into the designed instrument including (a) insert cartridge datum into locator slots in the instrument housing at an angle and (b) lower the cartridge into the slot and release the spring loaded clip to secure the cartridge.

\section{Results and Discussion}

Reaction Insert Design: The initial design intent for this refined cartridge was to transition all of the polypropylene components and films to polycarbonate, as 
polycarbonate is more robust and more commonly used for consumer products.However, preliminary studies showed that the double-layered reaction and pump pouch set-up used from the previous polypropylene cartridges could not be duplicated using polycarbonate film heat-sealed to a rigid polycarbonate body. The polycarbonate film is brittle and does not stretch, eliminating the flexible nature of the double-pouch system, which is necessary for the simple fluid handling and pumping mechanisms used for this cartridge. Therefore, the cartridge design was modified to retain the double-layered polypropylene reaction and pump pouch scheme as an independent component that is inserted into the polycarbonate cartridge body.

Valving and Fluid Handling within the Reaction Insert: The reaction insert within the cartridge needs to receive and contain the master-mix during the amplification process, then enable pumping of amplified master-mix onto the lateral flow strips. Fluid handling within the reaction insert (Figure 12a) is facilitated via a mechanism similar to a diaphragm pump, with a reaction pouch enclosed by a pump pouch, and including passive check valves at the inlet and outlet to the reaction pouch. An inlet valve of lower opening pressure prevents back flow leakage of the reagents. An outlet valve with higher opening pressure ensures proper fluid containment during the reagent injection into the reaction pouch and the isothermal amplification period. These check valves have to be low-cost and mass-producible, with predictable nominal opening pressures and low dead volume. Commercially-available valves do not meet these requirements. Therefore, we developed a novel, custom valve design ${ }^{63}$ (Chapter 3 ) to implement the fluidic control. The assembled valves were press fit into the reaction inserts. 
To characterize the fluidic control of master-mix entering and exiting the reaction pouch through these valves, we injected fluid into assembled reaction insert using a syringe pump, and monitored the pressure upstream of the inlet port to the reaction pouch. We also monitored the pressure in the filled and closed pump pouch as a surrogate for the pressure in the reaction pouch. As buffer fills the reaction pouch, no further stretching of the polypropylene film covering the reaction or pump pouches is required. However, at the beginning of the experiment, the reaction pouch is empty with the reaction pouch film pressed up against the dome of the reaction chamber, and the pump pouch is fully-filled. As the reaction pouch is filled, a similar volume of pump pouch fluid is forced out of the pump pouch outlet and pressurizes the enclosed ePump chamber. Therefore, we can assume that the pressure measured at the closed pump pouch outlet approximately reflects the pressure in the reaction pouch. For this experiment, four replicates all showed similar behavior, an example of which is given in Figure 14. Approximately $30 \mu \mathrm{L}$ of compressible air ahead of the liquid causes slow pressurization at the inlet at the start of the pumping. The inlet pressure then increased while the pump pouch pressure remained constant at zero, until the opening pressure of the inlet valve was reached. This was followed by a pressure drop as the fluid rushed into the reaction pouch. As the reaction pouch filled with liquid, the inlet pressure and pump pouch pressure both increased, but the difference between the two pressure measurements remained constant at around 4 PSI, which is the required pressure to sustain the inlet valve in the open position. Eventually, the opening pressure of the outlet valve was reached, and the fluid began to exit into the lateral flow detection chambers. The outlet valves opened at around 6 PSI pressure difference between the pump pouch / reaction pouch, and open atmosphere. In these four 
replicates, $250 \pm 60 \mu \mathrm{L}$ of reaction buffer could be pumped into the reaction pouch between the opening events at the inlets and outlet valves, which is more than adequate to accommodate the desired assay volume of $100 \mu \mathrm{L}$ per reaction.

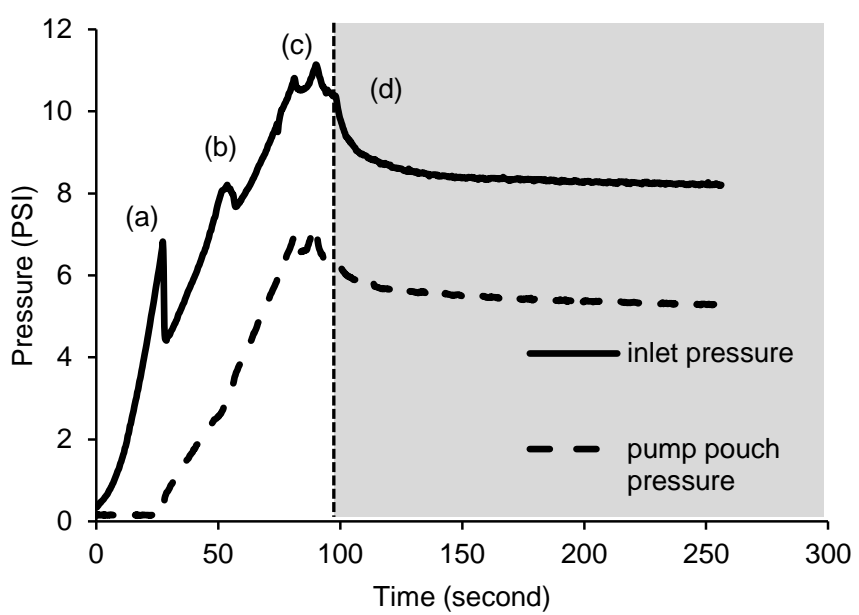

Figure 14: Fluidic control of reaction pouch filling. Fluid was pumped into the reaction pouch at $3 \mu \mathrm{L} / \mathrm{sec}$ for $91 \mathrm{sec}$, and then the pump was turned off (grey area). The inlet pressure was measured upstream of the inlet valve, in-line with the fluid pumping into the reaction pouch. The pump pouch pressure was measured as a surrogate to indicate the pressure inside the reaction pouch. (a) Inlet pressure increases, then drops, which indicates inlet valve cracking. (b) Inlet and pump pouch pressures build as fluid fills the reaction pouch. The outlet valve does not allow fluid to flow out of the reaction chamber. The small pressure peak presumably indicates dead air trapped in the reaction pouch exiting the chamber through the outlet valve. (c) Maximum peak pressure indicates outlet cracking pressure and the point at which fluid begins flowing out of the reaction chamber. (d) Inlet pumping was stopped, allowing fluid to exit the reaction chamber and pressure inside the reaction pouch to equilibrate. 
Hydrophobic Barriers and Venting: Proper venting of dead air stuck in the channels upstream of the reaction pouch is crucial to achieve reproducible amplification, as large gas bubbles introduced into the reaction pouch may interfere with heat transfer and temperature equilibration throughout the master-mix volume in the reaction pouch. We selected a PTFE porous membrane with $0.2 \mu \mathrm{m}$ pores on polypropylene mesh backing as venting material. This membrane was heat sealed on top of the polycarbonate channels immediately upstream of the reaction pouch to be used as a hydrophobic barrier to vent the trapped air.

We characterized the performance of the hydrophobic barrier by injecting $150 \mu \mathrm{L}$ reaction buffer fluid using a syringe pump into the cartridge sample input port and then into the reaction insert, while measuring the pressure in the upstream fluid channel (Figure 15).

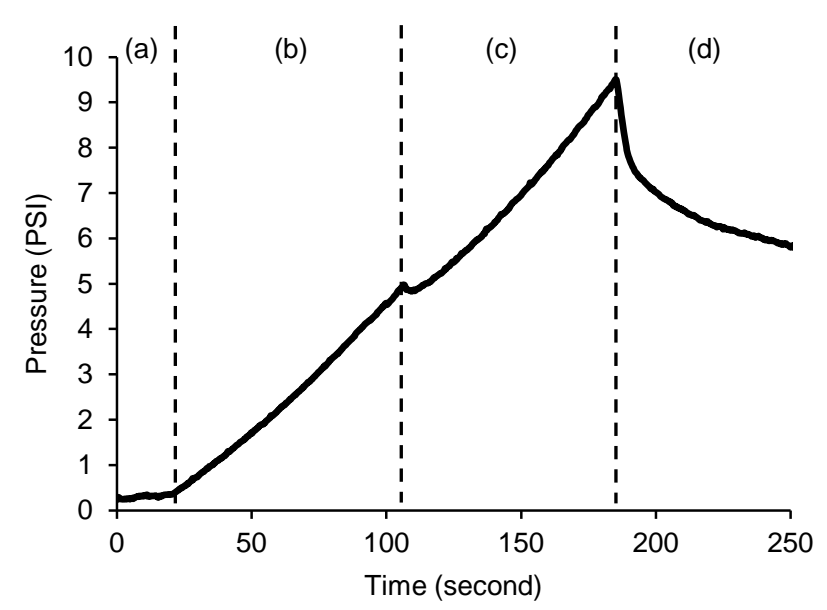

Figure 15: Pressure measurement in the upstream channel during injection of $150 \mu \mathrm{L}$ reaction buffer from the sample inlet port into the reaction insert. (a) The barrier vents the dead air ahead of the fluid until the fluid fills the upstream channel. No air is injected into the reaction pouch. (b) The venting barrier closes as liquid fills the area underneath the barrier. Pressure builds in the upstream fluidic channel until the inlet valve opens. (c) The 
fluid is pumped into the reaction pouch through the inlet valve. The channel pressurizes due to the pressure exerted by the filled and closed pump pouch. (d) After approximately $100 \mu \mathrm{L}$ liquid is injected into the reaction pouch, the gas behind the injected volume is vented through the venting barrier and the remainder of the volume is trapped in the vented portion. No air is injected into the reaction pouch.

This hydrophobic barrier vented the gas in the channels as fluid was pushed from the sample inlet port towards the reaction insert without pressure buildup (Figure 15a), but became impermeable once the liquid reagents reached the inlet valve of the reaction insert. At that point, the pressure increased until the inlet valve cracking pressure was reached (Figure 15b). Liquid reagents then entered each reaction pouch and, during this process, the pressure further increased because pressure was exerted onto the closed and filled pump pouch. However, the venting barrier did not allow fluid to leak out of the cartridge during the entire 10 PSI fluid pressure build-up encountered in this process (Figure 15c). Once approximately $100 \mathrm{uL}$ of the liquid reagents was injected into each reaction pouch, the barrier released the pressurized gas present in the channel upstream of the injected volume, effectively stopping the pumping action (Figure 15d), leaving approximately $50 \mathrm{uL}$ of reagents in the venting portion of the upstream channel. A thin layer of fluid wetted the porous membrane and, therefore, a small pressure in the venting channel was required to open the porous membrane. However, the decrease in the upstream pressure was sufficient to close the inlet valve and prevent further pumping of air into the reaction pouch. 
Lateral Flow Detection Chambers: For our cartridge design, it is particularly important that the lateral flow strip chambers be completely sealed, since the master-mix will have been amplified prior to being pumped into the chamber. For this refined amplification and detection cartridge, the lateral flow chambers needed to be integrated into the core polycarbonate body, requiring the chamber to be sealed with polycarbonate film. Previous investigations indicated that lateral flow strip performance can be negatively affected if pressure builds inside the lateral flow strip chamber as master-mix is pumped in and the air inside the chamber compresses. The polypropylene film used in the previous cartridge version (Chapter 2) could stretch to accommodate the increase in volume when the amplified master-mix was pumped into the lateral flow strip pouches, which is not ideal but proved to be sufficient for initial testing. To mitigate this problem, and since the polycarbonate film is less flexible than polypropylene film, we included commercially-available hydrophobic barriers (Qosina) to vent air out of the new lateral flow chambers, reducing any possible pressure build-up.

Additionally, the lateral flow strips in the previous amplification and detection cartridge design were approximately $1 \mathrm{~cm}$ shorter than standard sized strips (typically $\sim 5$ $\mathrm{cm}$ ), and had to be run in anti-parallel orientation to fit within the footprint of the top of the cartridge. For this refined amplification and detection subunit, the lateral flow chambers were re-designed to fit two full-length strips in parallel orientation. We investigated the fluid flow and lateral flow strip performance within chambers of several sizes, with varying additional features (Figure 16). One of these additional features was a reagent pool area located above the fluid conduit connecting the reaction chamber with the lateral flow strip chamber. We manufactured lateral flow strips wtih sample pads that 
extended into this reagent pool. We observed the best and most reproducible performance for lateral flow detection chambers that contained such a recessed reagent pool, which ensures that the lateral flow strip performance is not compromised by a rapid outflow of fluid from the reaction pouch, and which enables controlled absorption of the amplified master-mix into the lateral flow strip, allowing proper reconstitution of the dried reagents. Previous studies indicated that if the flow rate into the lateral flow strip chamber is too fast, then the fluid will flow around and over the lateral flow strip, instead of wicking through and across the nitrocellulose membrane.

These studies further demonstrated that the hydrophobic barrier needs to be recessed below the chamber bottom surface, that the largest chambers tested had the best fluid control, and that a pressure point at the interface of the conjugate pad and the nitrocellulos is needed for proper fluid flow along the lateral flow strip. This pressure point helps to guide the fluid as it wicks from the conjugate pad to the nitrocellulose membrane. In the current design, we apply pressure manually as the fluid is pumped from the reaction pouch into the lateral flow strip chamber. In future design iterations, however, this pressure point will need to be included in the chamber design.

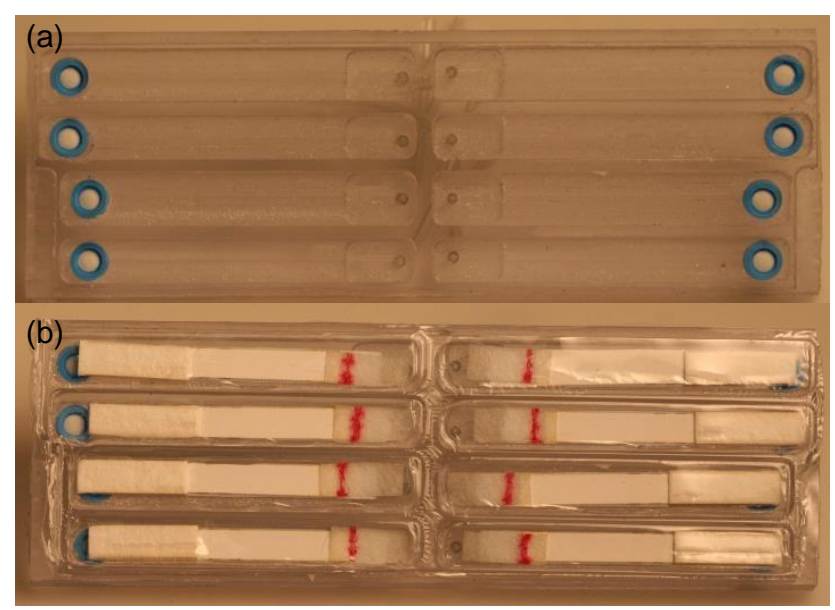


Figure 16: Lateral flow strip chamber testbed. (a) Empty lateral flow strip chambers, showing embedded hydrophobic barriers (blue). (b) Lateral flow strip chambers with lateral flow strips sealed by polycarbonate film. The red line on the conjugate pads is the lateral flow conjugate that has been dispensed and dried onto the conjugate pad.

Electrolytic Pumping: After the amplification reaction is completed, the integrated ePumps are used to push the amplified master-mix from the reaction pouch into the lateral flow detection chambers. The ePump chambers are filled with electrolyte solution, and are connected via a closed channel to the pump pouch port of the reaction insert. By applying a constant current to the electrodes in the ePump chambers, hydrogen and oxygen gas is generated though water electrolysis. This gas then pressurizes the silicone oil which fills the pump pouches. Since the pump pouches are supported from below by the instrument surface, the pressure is transferred to the reaction pouch through the flexible membrane separating both pouches. Once the outlet valve is opened, the membrane pushes the amplified product from the reaction pouch to the lateral flow detection chamber, at a flow rate dependent on the characteristics of the outlet valve and the pressure built up in the reaction pouch, which in turn is dictated by the rate of gas generated by the ePump. If the ePumps generate too much pressure, then the reaction pouch contents will be pushed too rapidly onto the lateral flow strips, exceeding the capacity of the lateral flow strip sample pad to absorb the liquid and properly reconstitute the reagents on the conjugate pad, which may negatively impact the detection readout. Our prototype instrument electronics contain controllable current sources to power the ePumps, and are capable of generating a constant current with a resolution of $2.7 \mathrm{~mA}$ up 
to $700 \mathrm{~mA}$. The lower end of this current range will be used to power the ePumps in the amplification and detection unit. We determined that by applying $8.2 \mathrm{~mA}$ or $13.7 \mathrm{~mA}$ of current to the ePumps, the outlet valve opened within 98 or 57 seconds, respectively, from the start of electrolysis. In both cases, the fluid exited the reaction pouch at a flow rate of approximately $2-4 \mathrm{uL} / \mathrm{s}$, which is in the required range to enable suitable lateral flow performance.

Thermal Control: A constant and controlled temperature throughout the reaction pouch, and reproducible heat transfer between the reaction pouch and instrument heater, is critical to the performance of this diagnostic system. To facilitate appropriate thermal control, the cartridge contains thermal insulation above the reaction insert, and the bottom of the reaction insert is designed to protrude below the bottom cartridge surface by 400 $\mu \mathrm{m}$. The pump pouches are completely filled with silicone oil and slightly inflated. Therefore, the liquid-filled pump pouches enclosing the reaction pouches are pushed up against the heater surface, ensuring good thermal contact between the heater and reaction pouches. 


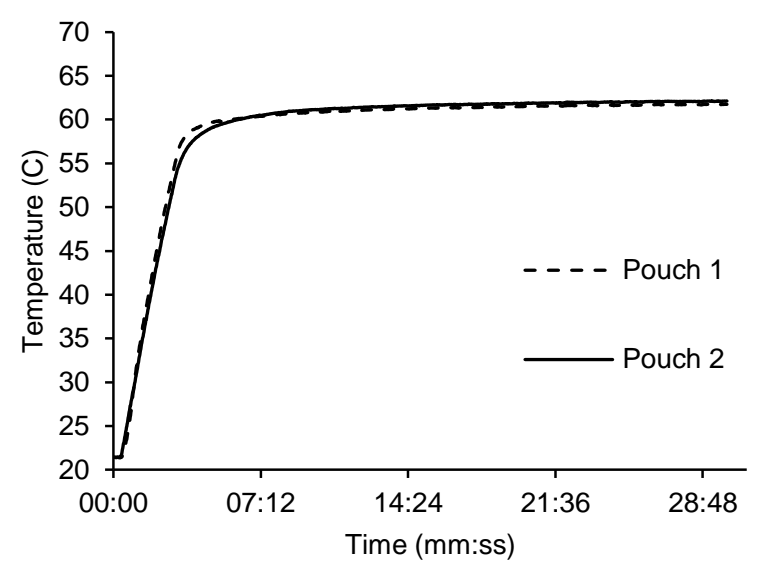

Figure 17: Temperature of the fluid inside the reaction pouch of a cartridge attached to the heater as a function of time. The fluid reaches a stable temperature within approximately ten minutes.

The combination of these strategies enables efficient heating of the reaction buffer within both reaction pouches to the targeted temperature within approximately 10 minutes (Figure 17). The temperature is maintained at steady-state over the duration of the isothermal amplification period. We implemented an established LAMP reaction which requires a constant temperature of $63{ }^{\circ} \mathrm{C}$ in our cartridge. However, the thermal set-point of the heater incorporated in our instrument can be readily adjusted to the desired temperature required for different types of isothermal amplification reactions.

Entire Process and Assay Execution: The process consists of the following steps: an empty cartridge is attached to the instrument, which is then powered on. At that point, the heater LED blinks red to indicate system initialization, and the system performs an ePump check: if proper connections between the instrument and ePumps are established, 
then the two blue ePump LEDs blink three times. The heater LED then turns solid red while the heater warms up to the programmed temperature. The heater LED turns solid green once the final heater temperature has been reached. At that point, the sample is manually injected into the reaction pouch. The master-mix remains in the reaction pouch chambers to incubate at the set temperature for the desired reaction time, at which point the heater is turned off and the reaction ePumps are turned on. Once suitable pressure is acheived within the pump pouches, the fluid in the reaction pouches will be forced through the outlet valves and into the lateral flow strip detection chambers.

(a)

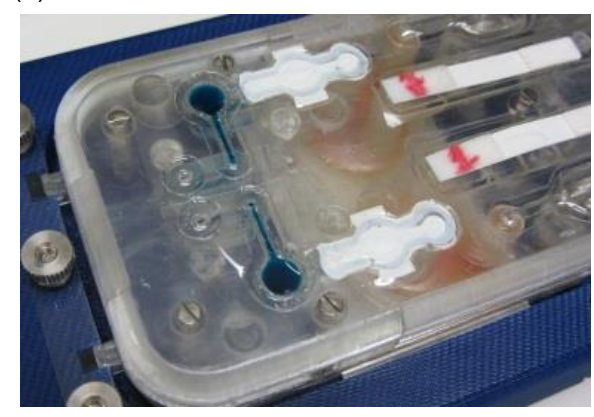

(b)

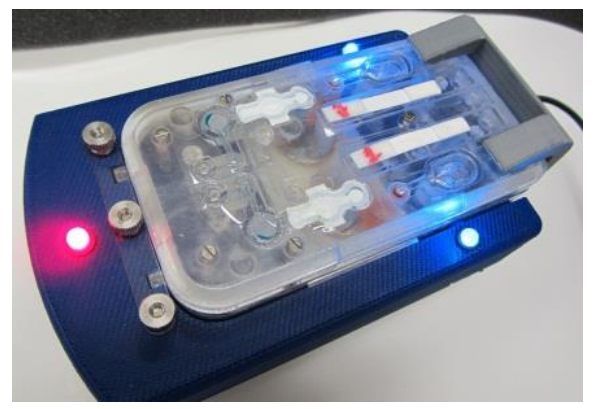

(c)

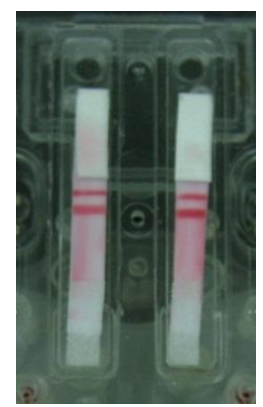

Figure 18: Demonstration of fluidic process in cartridge on instrument. (a) Cartridge attached to instrument, with reaction buffer (colored blue) injected into storage chamber. (b) Instrument LEDs indicate function of heater (red LED) and ePumps (blue LEDs). (c) Lateral flow detection of reaction buffer spiked with synthetic DNA product pumped out of the reaction pouch onto the lateral flow strips in the cartridge.

To demonstrate fluid handling in the cartridge, we injected $150 \mathrm{uL}$ dye-colored reaction buffer into each of the two sealed conical storage chambers (Figure 18a). We then manually triggered the instrument to start the amplification reaction cycle, and pumped the liquid reagents into the reaction pouch by pushing air at a rate of $3 \mathrm{uL} / \mathrm{sec}$ 
into the two sample inlet ports using a syringe pump. After suitable pressure had been reached to open the inlet valve, the reaction buffer moved downstream into each reaction pouch of the reaction insert. As the buffer entered the reaction insert, the pumping air advanced down the channel until it exited through the venting barrier. The release of upstream pressure closed the inlet valves. The injection process was completed in 2 minutes. This experiment omitted the heating and incubation steps to save time and, after the sample was injected, the instrument was triggered to apply $13.7 \mathrm{~mA}$ of current to the ePumps for 2 minutes, indicated by the ePump LEDs turning solid blue (Figure 18b). Within approximately 1 minute, sufficient pressure was reached in the pump pouches to open the outlet valves, and the contents of the reaction pouches were pushed into the lateral flow detection chambers in less than one minute. We also executed the process using uncolored reaction buffer spiked with a synthetic amplification product that can be detected on the lateral flow strip to verify that the cartridge enables a suitable visual readout (Figure 18c).

We then conducted an initial proof of principle experiment to demonstrate that a LAMP reaction can be executed successfully in the cartridge. This reaction is based on a published method to detect the gyrB gene within $M . t b$ genomic DNA, ${ }^{38}$ which we have modified to enable coupling to $\mathrm{NALF}^{80}$ (Chapter 2). To verify proper amplicon formation, our first experiment utilized gel electrophoresis rather than lateral flow detection. As described above, we turned on the instrument and attached an empty cartridge to the handheld unit, allowing at least 10 minutes for the heater and cartridge to reach appropriate reaction temperature. We then injected LAMP master-mix with 3000 copies of M.tb genomic DNA per reaction into the reaction pouches and allowed the reaction to 
incubate for 60 minutes. After incubation, amplified master-mix was withdrawn from the cartridge and analyzed via gel electrophoresis (Figure 19). Both reaction pouches generated the expected ladder of high molecular weight concatenated amplicons typical for LAMP, with banding patterns similar to control reactions that were performed in standard reaction tubes.

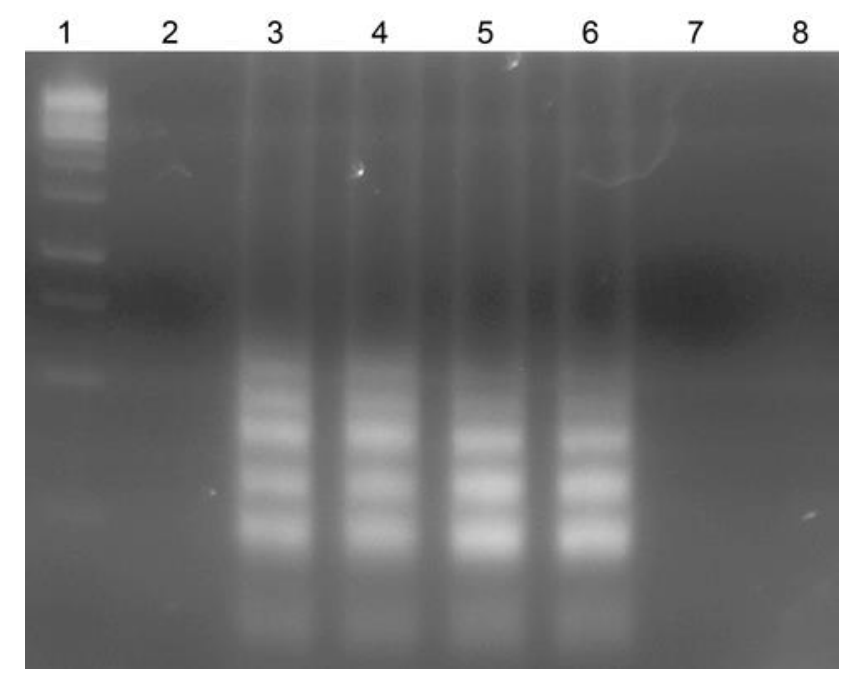

Figure 19: Detection of M.tb genomic DNA through 60 minutes of isothermal LAMP in the cartridge on the instrument, verified via gel electrophoresis of amplified master-mix removed from the cartridge after the reaction. Amplified product from both reaction pouches (lanes 3 and 4) generated a similar pattern as the positive control reactions performed in tubes on a standard heater (lanes 5 and 6), indicating that desired amplification had occurred in the cartridge. All positive reactions contained 3000 copies M.tb genomic DNA. No products were generated in negative control reactions (lanes 7 and 8) that did not contain M.tb DNA. Lane 1 contained $1 \mathrm{~kb}$ molecular weight ladder and lane 2 was empty. 
We have modified the lateral flow configuration from the previously reported configuration ${ }^{80}$ (Chapter 2) to rely on hapten-only interactions on both the test and controls lines. In this new configuration, neutravidin and rabbit anti-sheep antibody are immobilized onto the nitrocellulose for the test and control lines, respectively. A polyclonal digoxygenin antibody (anti-DIG) from sheep is conjugated to colored microspheres and will bind to the DIG-labeled BIP primer if the amplicon product is present in the sample. Additionally, the neutravidin that is immobilized on the nitrocellulose strip will bind to the biotinylated FIP primer if the amplicon product is present, resulting in a visual line in the test area. The rabbit anti-sheep antibody present at the control area binds the anti-Dig conjugate and will be present regardless of the presence of LAMP amplicon.

Initial studies using LAMP master-mix with 3000 copies of M.tb genomic DNA in positive samples have demonstrated proper automated fluid handling from sample insertion through lateral flow detection on board the cartridge. However, these initial positives samples did not show amplification on the lateral flow strips. We have identified possible causes for the lack of amplification. First, all prior studies were conducted with cartridges that had been manufactured in dirty lab conditions. While we were able to demonstrate amplification, there was a risk that these cartridges had been contaminated during manufacturing. We have since transitioned all cartridge manufacturing processes into clean lab conditions (Appendix G). Under these new conditions, the thermal bonding for all fluid conduits on the cartridge is performed in a laminar flow hood. When running the initial amplification and detection assays in these clean cartridges, we noticed the pump pouches showed leakages. It is possible that the air 
blowing over the heated arbor press in the laminar flow hood is preventing the arbor press from reaching the necessary temperature to provide sufficient thermal bonding. In addition, this arbor press may not consistently have even contact across the heated die and the cartridge surfaces, yielding one edge of the thermal bond ineffective. We are currently investigating strategies to mitigate this issue.

Moreover, the previous studies demonstrating amplification in the cartridges were performed by bypassing the upstream fluidic channels and chambers leading into the reaction pouch. For these studies, the LAMP master-mix was injected directly into the reaction pouches. For the current studies, the master-mix is now injected through the sample port, exposing the reaction mixture to a higher amount of plastic surface area in the upstream channels and chambers. We had demonstrated polycarbonate assay compatibility in earlier studies, but noted that these cartridges had been exposed to UV radiation while the insert-molded electrodes in the polycarbonate core were secured in place with UV glue. We then tested the irradiated polycarbonate for possible inhibition, but none was observed.

Finally, in our work with the initial cartridge prototype (Chapter 2), we observed that the cartridges required higher concentrations of BSA in the master-mix as compared to standard reaction tubes (Appendix F). We hypothesize that this increase in BSA concentration is necessary to mitigate non-specific adsorption of enzyme to the plastic surfaces in the cartridges. Therefore, with the increase in plastic surface area from the upstream fluidic channels and chambers in this refined cartridge prototype, we will investigate increasing the BSA concentration in the master-mix to possibly passivate these surfaces. 


\section{Conclusions}

We refined the amplification and detection prototype cartridge and instrument to perform the necessary fluidic and thermal control operations for automated isothermal nucleic acid amplification coupled to visual lateral flow detection. The system utilizes economical and scalable manufacturing techniques as a step toward point-of-care infectious disease diagnosis in low-resource settings. Additionally, we developed and implemented in this cartridge low cost fluidic components such as passive valves, electrolytic pumps, and hydrophobic vents. We have characterized and verified appropriate performance of the key fluid handling and thermal control components, have demonstrated that the system can execute the entire process sequence, and, in a preliminary experiment, have demonstrated the detection of 3000 copies of purified M.tb genomic DNA via isothermal LAMP amplification using this disposable cartridge and instrument. We have transferred the cartridge manufacturing to a clean environment to mitigate contamination risk, and have manufactured 10 cartridges in this environment. Preliminary tests have revealed technical issues with these cartridges, which we are currently addressing. We anticipate these issues will be resolved soon, rendering the refined amplification and detection subunit fully-functional. 


\section{CHAPTER 5: REAGENT STORAGE AND RECONSTITUTION}

\section{Introduction}

Point of care diagnostics intended for low-resource settings must be suitably designed for the targeted use environment. For example, many health care facilities in low-resource countries lack un-interrupted access to electricity, making reagent refrigeration impossible. Moreover, distribution to remote health care facilities cannot rely on coldchain transportation, since this infrastructure is nonexistent in the majority of these settings. Therefore, reagents need to be incorporated in the device in a stabilized form to withstand prolonged exposure to ambient and above ambient temperatures. Additionally, health care workers at these facilities have minimal training, and lack the required tools to precisely measure and combine reagents. Therefore, reagents need to be aliquoted and stored on-board the disposable cartridges.

Different reagent stabilization approaches for nucleic acid testing systems have been reported. In settings where refrigeration is available, master-mix reagents can be stored in reagent packs or pre-measured on-board cassettes in reservoirs or pouches. ${ }^{33,81}$ Lyophilization of master-mix reagents for recombinase polymerase amplification (RPA), ${ }^{82}$ and LAMP, ${ }^{82,83}$ plus commercially-available PCR beads (Illustra "PuRe Taq ReadyTo-Go PCR beads," GE Healthcare, Piscataway, NJ), ${ }^{84}$ have been demonstrated as a viable stabilization method. In most cases, excipients are added to the reagents to aid in preservation, such as trehalose, a protein-stabilizer. Additionally, to ensure proper consistency of the lyophilized material, a bulking agent, such as dextran, is often added. However, lyophilization of master-mix reagents cannot be performed on regular laboratory lyophilizers used to prepare purified peptides and proteins for storage. Master- 
mix lyophilization requires large systems with temperature-controlled reagent trays and programmable temperature and time ramps. Lyophilized pellets need to be generated with suitable consistency to enable manipulation. While this has been done, it is not trivial. Lyophilized pellets are very hydroscopic and often hard to handle, which complicates their integration into a final device.

Alternate reagent stabilization approaches have been developed, often with the addition of trehalose ${ }^{85}$ Other groups have reported using heat to stabilize reagents in microfluidic devices by drying at $37{ }^{\circ} \mathrm{C}^{86}$ or at $55{ }^{\circ} \mathrm{C} .{ }^{87}$ Kiviniemi et al. have reported using a combination of vacuum- and air-drying to stabilize PCR reagents. ${ }^{88}$ Paraffinpassivation is another method of stabilizing reagents on-board microfluidic chips, in which reagents are added during assembly and then encapsulated in paraffin wax. ${ }^{89}$ Glassification ${ }^{90}$ is another method of reagent stabilization used in commercial products which includes the addition of a glass-forming filler material, such as glucose, sucrose, maltose or maltotriose, to produce material that is stable at room temperature using freeze-drying ${ }^{91,92}$ or vacuum-drying. ${ }^{93}$

During use, master-mix reagents in liquid, lyophilized, or dried form need to be reconstituted and combined with purified DNA present in the sample preparation eluate to yield the final master-mix. Effective mixing of master-mix reagents before nucleic acid amplification is important, because reaction kinetics are affected by reagent concentrations. Assay performance may thereby be negatively affected if the reagent concentrations vary within a reaction volume due to improper mixing. Active mixing of master-mix components in cartridges designed for nucleic acid testing has been reported using plungers (Cepheid GenXpert) and pistons (Rheonix CARD), but these approaches 
require additional hardware, thereby increasing the system cost and complexity. Passive mixing by diffusion is a viable option if the diffusion length is held to a minimum and the reaction volume can be contained in a single chamber for a set period of time to allow for diffusive mixing to reach the final equilibrium.

The refined amplification and detection cartridge described in Chapter 4 was designed to hold a lyophilized master-mix pellet in a conical chamber (Figure 20). We generated lyophilized LAMP reagent pellets suitable for integration into this chamber in collaboration with, and using the state-of-the-art lyophilization equipment at, the Program for Appropriate Technology in Health (PATH, Seattle, WA). These pellets produced promising amplification results when reconstituted and amplified in standard PCR tubes. However, on the cartridge, we found that the reconstitution process did not enable uniform and effective mixing. The cartridge design assumes that the eluate from the sample preparation module is pumped into the conical chamber, where it re-hydrates the master-mix pellet. After incubating the liquid in this chamber for a suitable time to ensure uniform passive mixing, the final reaction mixture would be pumped into the reaction pouch. In reality, the initial fluid front entering the conical chamber was quickly wicked into the pellet, drawing a small volume out of the upstream fluid channel and introducing an air bubble into the fluid path (Figure 20b). This air bubble prevented the reaction fluid from mixing as one continuous volume. Subsequent pumping to move the liquid out of the conical chamber led to the initial fluid front containing highly concentrated reconstituted reagents exiting first (Figure 20(ii)), followed by an air bubble, and a second volume with much lower reagent concentration (Figure 20b(i)). Although the air bubble can be removed through the hydrophobic barrier, the liquid entering the reaction 
pouch was not well mixed, which would compromise controlled isothermal amplification. This lack of fluid control and mixing when reconstituting the master-mix reagents made this lyophilized pellet and conical reconstitution chamber embodiment incompatible with our cartridge design.

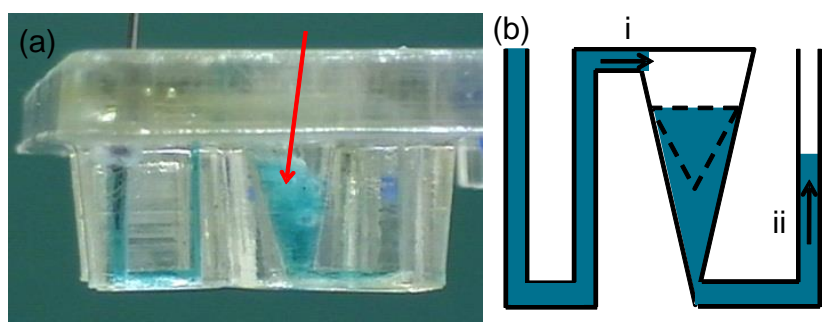

Figure 20: Lyophilized pellet storage and reconstitution chamber as originally designed.

(a) Reconstitution of pellet (red arrow) in blue colored water. (b) Depiction of reconstitution, illustrating the pellet wicking the initial fluid front to the bottom of conical chamber and pumping further downstream, while introducing an air bubble in the fluid stream separating the initial fluid front (ii) from the remaining fluid (i).

As previously discussed, our ultimate goal is to develop a fully-integrated system that performs sample preparation, amplification, and detection. The work described in this thesis pertains to the module for nucleic acid amplification and detection, developed at KGI. In a parallel effort, Claremont BioSolutions has developed a disposable cartridge and electronics instrument system for sample preparation and nucleic acid extraction. The next step toward our overall project goal is to combine these two separate cartridges into one fully-integrated, sample-in to answer-out cartridge. In this fully-integrated cartridge, the eluate exiting the upstream sample preparation subunit needs to reconstitute the stabilized master-mix reagents before it can be pumped into the downstream reaction pouch chamber for isothermal amplification. Ensuring the function of this fluidic 
interface between the sample preparation and amplification and detection modules is critical for the overall project effort. Therefore, we decided to develop and demonstrate suitable performance of a reagent storage and reconstitution method within a separate fluidic module, designed to act as the interface between the sample preparation module, and the amplification and detection module (Figure 21).

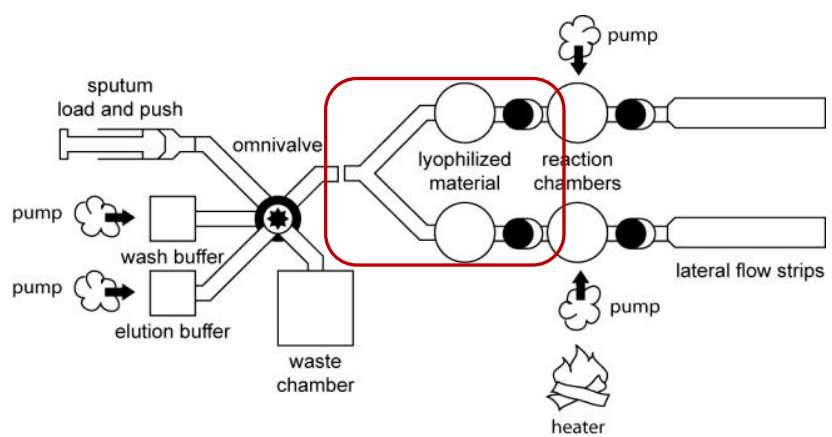

Figure 21: Original system overview diagram. Red area highlights the fluidics interface between sample preparation and amplification and detection, which needs to store stabilized master-mix reagents until they can be reconstituted by purified sample preparation eluate and pumped into the reaction pouch chamber. The initial design incorporated lyophilization to stabilize the master-mix reagents.

\section{System Overview}

The goal for the fluidic interface module is two-fold: (1) to provide a mechanism for storing dry master-mix reagents in a stabilized form, and (2) to provide a mechanism by which master-mix reagents can be reconstituted with purified sample preparation eluate, resulting in a homogeneous master-mix which then can be pumped downstream into the reaction pouch.

The fluidic interface module design is based on a novel reagent stabilization approach, utilizing master-mix reagents lyophilized onto a microfiber material. This 
material, in the form of a thin filter paper, was incorporated inside an elliptical fluid chamber, with an inlet and outlet conduit at the respective ends (Figure 22). Purified sample preparation eluate is pumped into the chamber through the inlet, first absorbing into the microfiber material containing dried master-mix reagents and then filling the chamber completely. The pumping is stopped, to allow the master-mix reagents to diffuse into the fluid. The master-mix is then pumped through the outlet, past a hydrophobic venting barrier and through a one-way valve into the reaction pouch chamber where amplification is initiated.

(a)

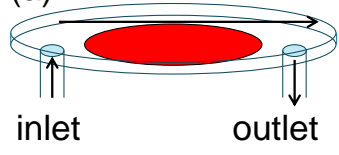

(b)

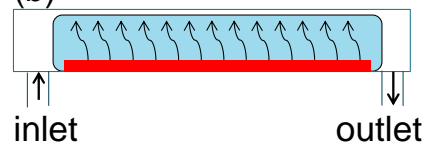

Figure 22: Fluidic system overview. (a) Top view: purified sample enters the chamber, first absorbing into the lyophilized master-mix material matrix, and then filling the chamber completely. (b) Side view: after a short incubation period to allow the reagents to diffuse, the reconstituted reaction master-mix is pumped through the outlet.

\section{Experimental}

Fabrication: All testbeds were manufactured from solid pieces of polycarbonate plastic. Using a CNC mill, we machined elliptical chambers $(5-11.5 \mathrm{~mm}$ wide, $7.5-15 \mathrm{~mm}$ length, $.5-1 \mathrm{~mm}$ depth), in some cases containing additional internal features, with through holes at the ends of the ellipse serving as inlet and outlet conduits. Microbore tubing (polyvinyl chloride, $0.5-1 \mathrm{~mm}$ inner diameter, $1.5-1.8 \mathrm{~mm}$ outer diameter) was press-fit into the inlet and outlet fluid conduits (Figure 23). Some chambers contained additional fluid conduits and features, as discussed below. The reconstitution chambers 
and all additional fluid conduits on all testbed embodiments were sealed closed using PCR film.

We integrated a refined venting chamber into this fluidics interface module to expel any dead air trapped in the fluid channels prior to introducing the master-mix into the reaction chamber. Early testbed embodiments (Figure 24) utilized commerciallyavailable hydrophobic barriers (Qosina), while the final refined version incorporated custom venting barriers manufactured from a polypropylene-backed PTFE filter membrane, previously described in Chapter 4 . When using custom venting barriers, the filter membrane was heat-sealed onto the polycarbonate testbed using the heated arbor press. For the refined design (Figure 25), the outlet of the reconstitution chamber is connected to the inlet of the venting chamber via a fluid channel on the underside of the testbed. The outlet of the venting chamber is connected to the polypropylene reaction insert through a boss that protrudes from the underside of the testbed.

Fluidics Testing of Microfiber Materials: We tested several possible microfiber materials as a matrix onto which the master-mix reagents can be dried (Table 7). Using the testbed embodiment shown in Figure 23(a), a piece of microfiber material cut into the appropriate elliptical shape was placed in each chamber, which was then sealed closed using PCR film. We determined the volume of unrecoverable fluid inside the chamber containing the microfiber material in the following manner: a liquid plug of $\sim 150 \mu \mathrm{L}$ was injected using a syringe into the inlet tubing upstream of the reaction chamber. The specific volume of fluid was determined by measuring the length of the fluid plug in the inlet tubing, and then calculating the total volume using the inner diameter of the tubing. 
The liquid plug was then manually pushed into the reconstitution chamber, where it was incubated for $\sim 1 \mathrm{~min}$. The fluid was then manually pushed out of the chamber and into the outlet tubing, at a flow rate of approximately $100 \mu \mathrm{L} / \mathrm{min}$. The amount of fluid recovered from the reaction chamber was determined again by measuring the length of the fluid plug in the outlet tubing, and then calculating the volume based on the inner diameter of the tubing.

Table 7: Key dimensions and physical parameters for support materials.

\begin{tabular}{|c|c|c|c|}
\hline Product Name & Material & $\begin{array}{l}\text { Typical Thickness } \\
(\mu \mathrm{m})\end{array}$ & $\begin{array}{l}\text { Water Absorption } \\
\left(\mathrm{mg} / \mathrm{cm}^{2}\right)\end{array}$ \\
\hline $\mathrm{CF} 1^{\mathrm{a}}$ & $\begin{array}{l}100 \% \text { cotton linter, } \\
\text { light, thin grade }\end{array}$ & $175 \pm 26$ & 16 \\
\hline $\mathrm{GF} / \mathrm{A}^{\mathrm{b}}$ & Unbound glass microfiber & 271 & 98 \\
\hline $\mathrm{CF}^{\mathrm{a}}$ & $\begin{array}{c}100 \% \text { cotton linter, } \\
\text { medium weight }\end{array}$ & $335 \pm 49$ & 31 \\
\hline Fusion $5^{\text {a }}$ & $\begin{array}{c}\text { Proprietary FUSION material, } \\
\text { single-layer matrix }\end{array}$ & $350 \pm 40$ & 40 \\
\hline
\end{tabular}

${ }^{\mathrm{a}}$ GE Healthcare. ${ }^{\mathrm{b}}$ Whatman, a division of GE Healthcare.

Stabilizing Master-Mix Reagents: The reagent stabilization studies presented herein focused on developing a dry master-mix formulation for the LAMP M.tb assay discussed in Chapters 2 and 4. Elliptical pieces of microfiber material were pre-treated by dipping them into a solution containing $2 \%$ trehalose and $0.4 \mathrm{mg} / \mathrm{mL} \mathrm{BSA}$. The pieces were then placed inside standard $1.5 \mathrm{~mL}$ tubes, and were allowed to dry overnight at ambient temperature.

Next, $\sim 30 \mu \mathrm{L}$ of master-mix reagents ( $5 \mathrm{X}$ concentrated) were then pipetted onto the pretreated pads inside the $1.5 \mathrm{~mL}$ tubes. The tubes were then placed inside a Savant 
Speed Vac Concentrator, which was attached to a Labconco Freeze Dry System/Freezone 4.5. During the drying process, the rotor of the Speed Vac containing the samples was spun at 1300-1600 RPM, while the Labconco lyophilizer was pulling a vacuum of $\sim 0.1$ torr, with the cooling coil inside the lyophilizer set to $-40^{\circ} \mathrm{C}$.

The $5 \mathrm{X}$ concentrated master-mix solution contained $2 \%$ trehalose and other standard reaction buffer components (Tris, $\left.\mathrm{KCl},\left(\mathrm{NH}_{4}\right)_{2} \mathrm{SO}_{4}\right)$. For initial studies, only one of the more sensitive master-mix reagents (primer oligonucleotides, dNTPs and Bst polymerase) was added to the concentrated master-mix at a time. For reconstitution, the remaining LAMP master-mix reagents that were not included in the drying process were combined into a $150 \mu \mathrm{L}$ volume. The microfiber pieces containing the dried reagents were incubated in this reconstitution volume in a $1.5 \mathrm{~mL}$ tube, to enable the dried mastermix reagents to be released from the microfiber matrix. After incubating, the fluid was withdrawn from the tube and transferred to standard PCR tubes, followed by isothermal amplification on a Bio-Rad OpticonII real-time fluorescence PCR thermocycler, set to a constant temperature of $63^{\circ} \mathrm{C}$.

\section{Results and Discussion}

Reconstitution Chamber Design: The reconstitution chamber shape was designed to enable uniform filling of the chamber with incoming liquid, and to maximize fluid recovery out of the chamber after the master-mix reagents have reconstituted. There are two main mechanisms that prevent fluid from being recovered from the reconstitution chamber when air is pumped into the chamber: (1) fluid can be left behind in "free space" once the air has navigated a path from the inlet to the outlet of the chamber, and (2) fluid can be retained inside the microfiber material matrix. 
We selected an eliptical chamber shape approximately $11 \mathrm{~mm}$ wide, $15 \mathrm{~mm}$ long, and $1 \mathrm{~mm}$ deep, which provided a sufficiently large internal volume, and enabled reasonably uniform fluid filling and emptying due to the absence of sharp features. Based on related discussions with microfluidics experts, we investigated internal features for the reconstitution chamber, such as pillars and chevrons, to facilitate fluid mixing and to trap any air bubbles formed in the chamber (Figure 23). However, initial studies determined that sharp edges and/or topographical features inside the chamber lead to non-uniform chamber filling as well as the introduction of air bubbles and large dead volumes that could not be recovered when the fluid was pumped out of the chamber. Therefore, all future experiments were performed without additional structures within the chamber. 
(i)

(ii)

(iii)

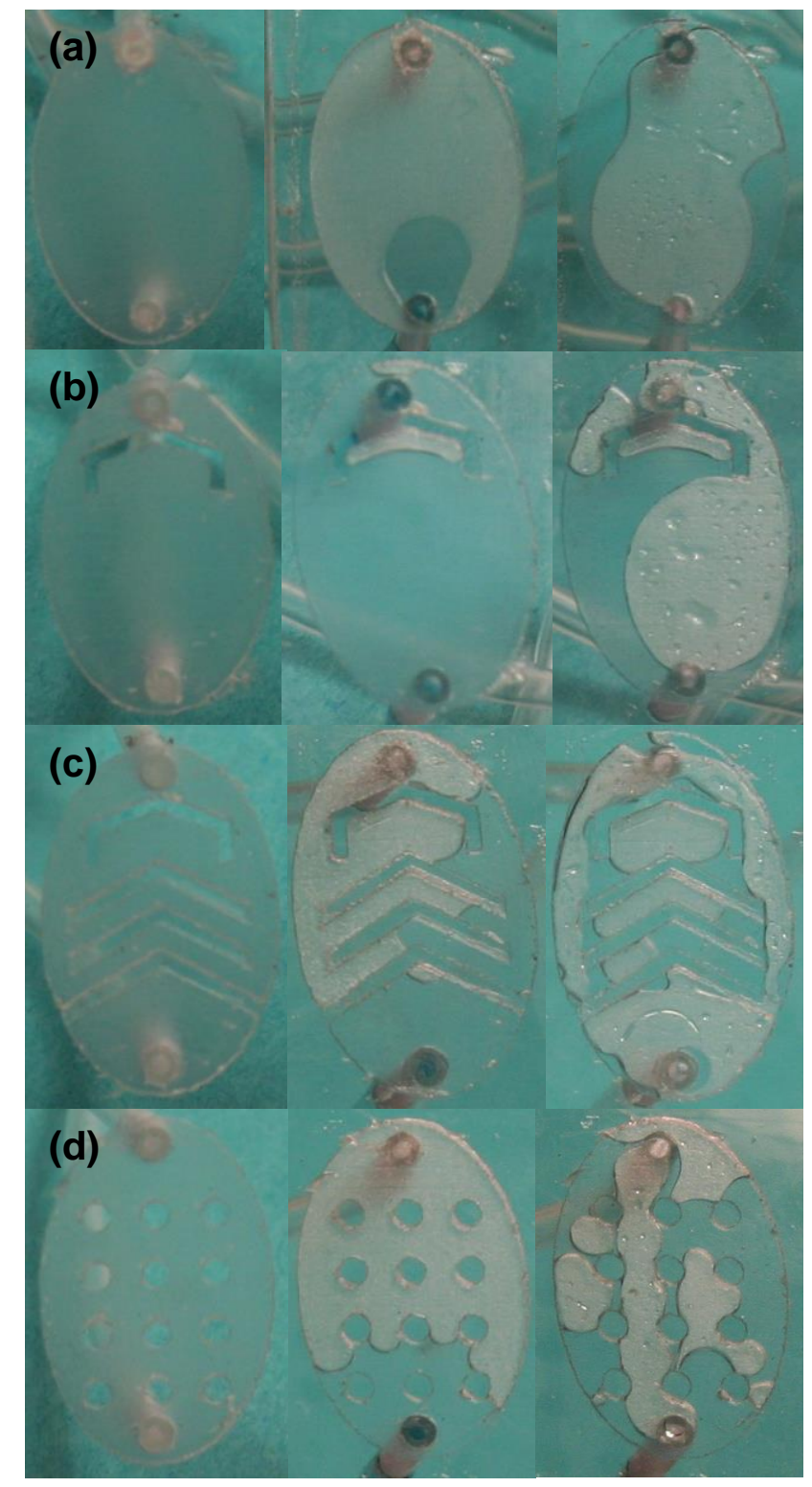

Figure 23: Investigation of different reconstitution chamber geometries. For each case, showing (i) empty chamber, (ii) partially filled chamber, and (iii) chamber after as much fluid could be emptied as possible. (a) Ellipse without any structures. (b) Ellipse with structure in front of outlet intended to catch any air trapped in the chamber. (c) Ellipse with chevron structures intended to enhance mixing in the chamber. (d) Ellipse with cylindrical pillars intended to enhance mixing in the chamber. All structures inhibited 
emptying of the chambers, leaving large dead volumes of unrecoverable fluid in the chambers. The empty ellipse shape (a) had the largest fluid recovery.

We observed that, even for the chambers without internal features, when emptying the chambers with an air chase, the amount of fluid left behind was irreproducible. As soon as the air pushed into the chamber behind the liquid found a path to the outlet, all remaining fluid in the chamber was unrecoverable. We hypothesize that this behavior is related to the properties of the chamber geometry and the inclusion of the cotton microfiber material into the reconstitution chamber. The Hele-Shaw cell is a wellcharacterized model investigating viscous fluid confined between closely spaced parallel sheets of glass. Experiments have shown that instability develops at the interface between fluids of different densities, resulting in round-ended "fingers" of less dense fluid penetrating the more dense fluid. ${ }^{94-96}$ If we assume that the reconstitution chamber can be modeled as a Hele-Shaw cell, this would explain the "fingering" seen in the empty chamber experiments where the air finds a path from the inlet to the outlet, leaving unrecoverable fluid in the chamber. Additionally, Hele-Shaw would suggest that there will always be unrecoverable fluid in the empty chamber.

However, introducing the cotton microfiber pad into the reconstitution chamber disrupts this system in such a way that the "finger" mechanism is no longer the driving force. Experiments have shown that, as the surface-tension affects become more important, the "finger" widens to fill the channel. ${ }^{97}$ Focusing on the reconstitution chamber shown here, the cotton pad is more hydrophilic than the polycarbonate in the empty chamber case. Therefore, with the inclusion of the cotton pad, the surface tension 
between the water above and the water inside the cotton pad becomes significant, resulting in the incoming air "finger" widening to expel all the water in the space above the cotton pad.

Volume Requirements: In our cartridge design (Chapters 2 and 4), a minimum of $75-$ $100 \mu \mathrm{L}$ of amplified master-mix needs to be pushed out of the reaction chamber and onto the lateral flow strips to ensure optimal performance, since we do not employ a separate lateral flow strip running buffer. This volume serves as the minimum required amount of fluid that needs to be recovered from the reaction pouch chambers. Accounting for additional dead volumes in the valves and fluid conduits leading into and out of the reaction chamber range ( $10 \mathrm{uL})$, and the dead volume underneath the hydrophobic venting barrier ( $~ 25 \mathrm{uL}$ in the refined design), we can estimate approximately $50 \mu \mathrm{L}$ of reaction fluid lost in the fluid conduits downstream from the reconstitution chamber. Therefore, we need to recover $\sim 150 \mu \mathrm{L}$ of reconstituted master-mix from the reconstitution chamber to ensure proper performance of downstream processes.

The original fully-integrated cartridge design entailed splitting the purified sample preparation eluate into two reaction chambers. Assuming no fluid losses in the reconsititution chamber, this would require at least $300 \mu \mathrm{L}$ of sample preparation eluate. Initial studies have indicated that the sample preparation module comfortably can yield $200 \mu \mathrm{L}$ eluate, but $300 \mu \mathrm{L}$ would be very difficult to obtain reproducibly. It further is unrealistic to assume no fluid losses in the reconstitution chamber. For this and other reasons related to overall size, cost, and complexity, we recently decided to simplify the fully-integrated cartridge system to contain only one reaction chamber. Therefore, if 150 
$\mu \mathrm{L}$ can be recovered out of the reagent reconstitution chamber after pumping in $\sim 200 \mu \mathrm{L}$ of purified sample preparation eluate, a loss of $25 \%$ (50 $\mu \mathrm{L}$ ) of input volume can be tolerated. The studies on the fluidic module shown here were performed using $150 \mu \mathrm{L}$ for the input volume, based on the original splitting design. Additionally, master-mix reconstitution in the reagent chamber should ideally be performed in batch mode, meaning that the entire volume of eluate (originally $150 \mu \mathrm{L}$, for future studies $200 \mu \mathrm{L}$ ) is introduced into the chamber at one time, and can be incubated above the pad to facilitate uniform reconstitution of the dried reagents, and diffusive mixing within the relatively short vertical distance above the pad.

Fluid Recovery: As previously described, fluid recovery depends on the amount of fluid left behind in "free space", as discussed in a previous section, and fluid retained inside the microfiber material matrix. For optimal fluid recovery, the microfiber matrix should absorb only a small amount of liquid. However, the microfiber material needs to be able to retain the necessary volume of reaction master-mix reagents during the drying process: $30 \mu \mathrm{L}$ of $5 \mathrm{X}$ master-mix, assuming a total volume of $150 \mu \mathrm{L}$, or $40 \mu \mathrm{L}$ of $5 \mathrm{X}$ master-mix, assuming a total volume of $200 \mu \mathrm{L}$. The requirements for fluid recovery when the reconstituted master-mix is pushed out of the chamber, and fluid retention of master-mix during drying have to be balanced against each other.

All of the microfiber materials were tested to determine the unrecoverable fluid lost in the material when approximately $150 \mu \mathrm{L}$ reaction buffer was pumped into the reconstitution chamber, followed by approximately $700 \mu \mathrm{L}$ of air (Table 8). The amount and percentage of unrecoverable fluid appears to depend on the material properties, and 
was most favorable for the two cotton based materials, CF1 and CF3, with a higher recovery for the thinner $\mathrm{CF} 1$ pad. The Fusion 5 and GF/A microfiber materials had the highest unrecoverable volumes. The CF1 material was chosen for future studies.

Table 8: Average fluid loss for each microfiber material

\begin{tabular}{ccc}
\hline Product Name & Average Fluid Loss $(\mu \mathrm{L})$ & Average Fluid Loss $(\%)$ \\
\hline CF1 & 25 & 15 \\
GF/A & 55 & 35 \\
CF3 & 41 & 24 \\
Fusion 5 & 67 & 47 \\
\hline
\end{tabular}

Venting Chamber: After reconstitution, the fluid leaving the chamber must pass a hydrophobic barrier to vent the dead air, thereby minimizing the amount air that is introduced into the reaction pouch. The original amplification and detection subunit design included a commercially-available hydrophobic barrier (Qosina) as a venting barrier, which we integrated into an early testbed designed to test the venting mechanism (Figure 24a). We demonstrated that trapped dead air could be vented through this barrier. However, once the microfiber material was added into the reconstitution chamber (Figure $24 b)$, the unrecoverable volume increased dramatically, which is undesirable. Therefore, a new venting chamber was designed.

In previous studies (Chapter 4), we verified that a polypropylene-backed PTFE membrane could be heat-sealed onto polycarbonate fluid channels to create a hydrophobic barrier that vents air through the top seal. ${ }^{62}$ Utilizing this design, we integrated the hydrophobic barrier on the top of a separate venting chamber, which was added downstream of the reconstitution chamber (Figure 24c). We heat-sealed the 
polypropylene-backed PTFE membrane around the perimeter of the venting chamber, and were able to demonstrate that all dead air was removed, and that the fluid exited the venting chamber as one complete bolus.

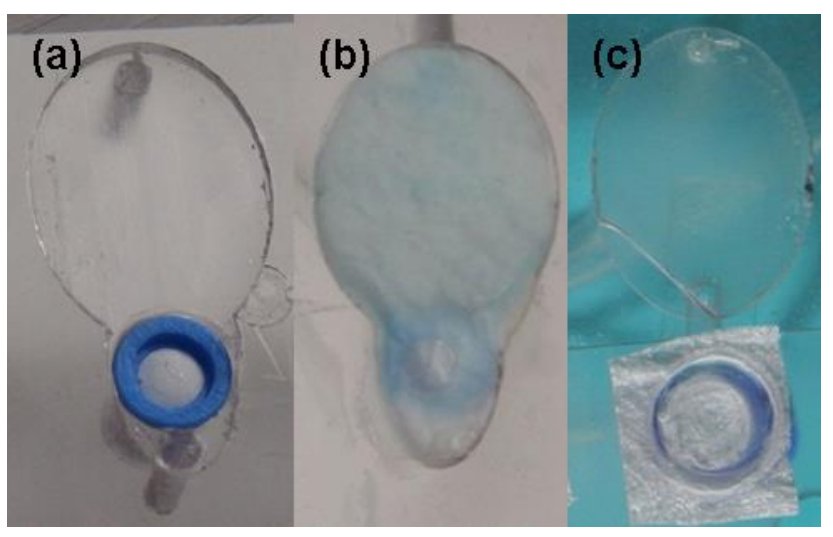

Figure 24: Reconstitution chambers with integrated venting barriers. (a) Reconstitution chamber with commercially-available hydrophobic barrier integrated into chamber. (b) Reconstitution chamber with integrated hydrophobic barrier and microfiber material, which had a higher percentage of fluid that could not be recovered from this chamber design. (c) Polypropylene-backed PTFE membrane material heat-sealed to an isolated venting chamber downstream of the reconstitution chamber.

Initial Fluidics Testing: We designed a final testbed to demonstrate that the fluidics module containing the reconstitution and venting chambers could interface directly with the polypropylene reaction insert (Figure 25). Since the current polypropylene inserts contain two reaction pouches per insert, the testbed was designed with two reconstitution and venting chamber sets, enabling duplicate fluidic tests per testbed. This testbed contained protruding bosses on the underside of the polycarbonate to mate the outlets of each venting chamber with the female luer-type fluid conduit on the inlets of the polypropylene reaction insert. The integrity of the liquid-tight seal was tested by inserting 
the boss into the polypropylene insert, and pumping fluid through the fluid conduit interface. We demonstrated that, while the conduit interface was not air-tight, it did not leak any fluid, making it appropriate for continuing forward with integration studies. We further demonstrated appropriate fluid handling in the testbed using a CF1 microfiber pad onto with food coloring was dried, to visualize the reconstitution process. Approximately $150 \mu \mathrm{L}$ of water was pumped into the reconstitution chamber, and the pumping was stopped when the chamber was full. The fluid was allowed to incubate for $\sim 2$ minutes above the pad, reconstituting most of the dried food coloring. After incubating, air was pumped into the reconstitution chamber, pushing the fluid through the venting chamber, to release any dead air, and then into a reaction pouch through a oneway, passive valve. The testbed performed as expected, with the fluid reconstituting the food coloring and the reaction pouch filled with colored water without air bubbles (Figure 25).

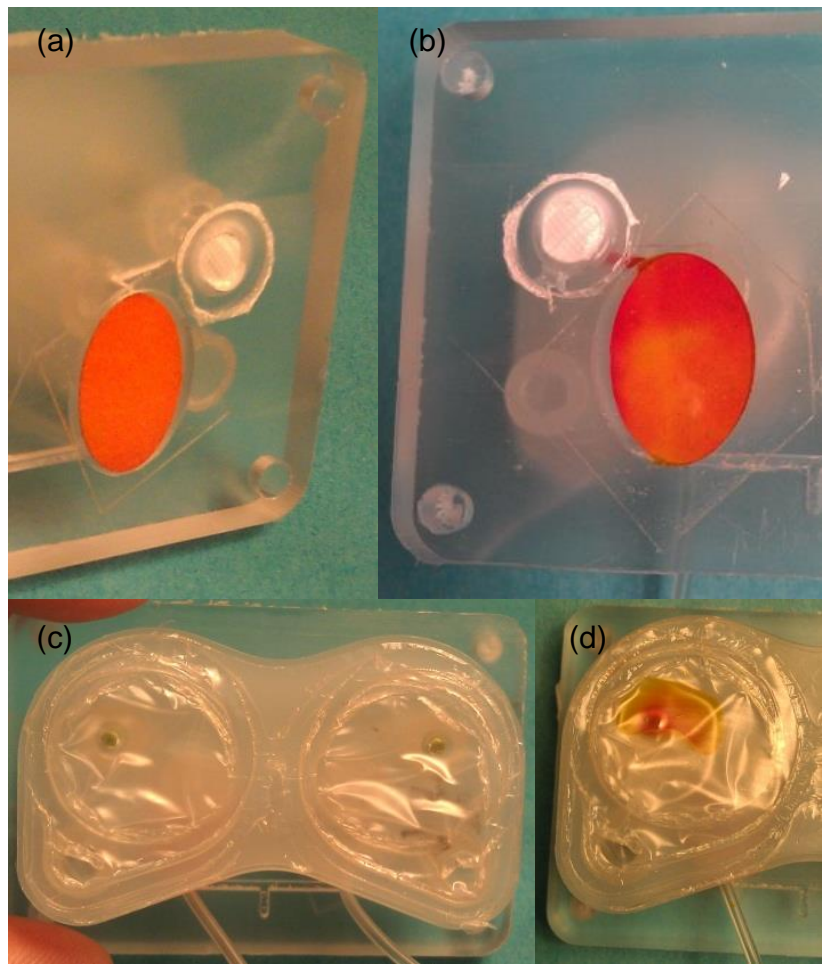


Figure 25: Fluidic interface testbed demonstrating the reconstitution of dried food coloring. (a) Top side of fluidic interface testbed showing one of the two fluidic module testbeds, including the venting chamber (white) and the reconstitution chamber, with orange food coloring dried onto the CF1 microfiber material. (b) Reconstitution chamber filled and reconstituting the orange food coloring, with fluid beginning to pump through the fluid conduit on the underside of the testbed from the reconstitution chamber to the venting chamber. (c) Bottom side of fluidic interface testbed, showing polypropylene reaction insert with empty reaction and pump pouches. (d) Reaction pouch with orange colored water inside after reconstituting the food coloring and pumping through the oneway valve into the reaction pouch chamber.

Reagent Stabilization: Our reagent stabilization approach used a modified lyophilization process has not been previously reported, and we are continuing to optimize this protocol. Current studies are focusing on drying the LAMP reagents onto the microfiber material. We have dried the primer oligonucleotides and the dNTPs individually, and in combination with each other, on the CF1 microfiber material. We were able to demonstrate amplification upon reconstitution (Figure 26). Amplification of negative samples does occur and is most likely due to amplicon contamination during the drying process. We are continuing to optimize the drying protocol to improve reconstitution performance. 


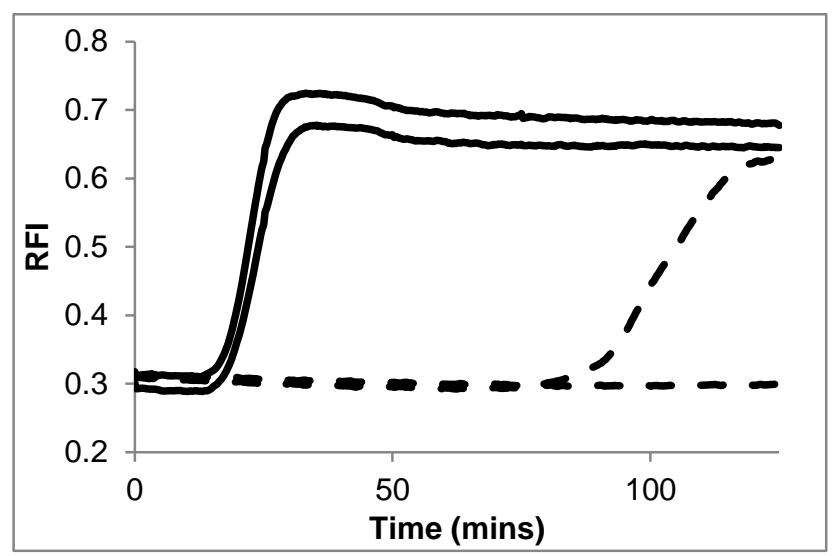

Figure 26: Reconstitution of dried LAMP master-mix reagents. Black solid line is reconstituted LAMP master-mix spiked with $M . t b$ genomic DNA (positive). Black dashed line is reconstituted LAMP master-mix without $M . t b$ genomic DNA (negative). Amplification of negative sample is most likely due to lab amplicon contamination affecting the reagent drying process.

Additionally, we reconstituted the dried master-mix material in the fluidic testbed to demonstrate amplification of reconstituted materials in the polypropylene reaction pouch insert (Chapter 4) on the original handheld heater instrument (Chapter 2). The reconstituted material was incubated in a reaction pouch insert on the handheld heater for one hour, and was then withdrawn via syringe to verify amplification through gel electrophoresis (Figure 27). As shown in Figure 27: Amplification of reconstituted master-mix materials. (a) Amplification results from two samples reconstituted in the fluidics testbed, with M.tb genomic DNA spiked into the reconstitution buffer. Testbed \#1 shows amplification while Testbed \#2 does not. (b) Amplification results from samples reconstituted in standard tubes and amplified on a bench-top thermocycler. The reconstituted NTC sample did not contain M.tb genomic DNA but does show 
amplification, likely due to amplicon contamination of lab areas.Figure $27 \mathrm{a}$, one of the reconstituted samples showed amplification on the gel similar to the reconstitution control reactions which were reconstituted in standard tubes and amplified on the benchtop thermocycler. These initial reconstitution results are promising, and we are continuing to optimize the reconstitution process in the fluidic testbed. Figure $27 \mathrm{~b}$ shows the amplification of samples reconstituted in standard tubes, with both the positive and negative reactions indicating clear amplicon product. Most likely the amplification of the negative reaction is due to lab contamination of amplicon product, and we are working toward setting up a clean work area to improve the drying protocol and to reduce false positive reconstitution results.

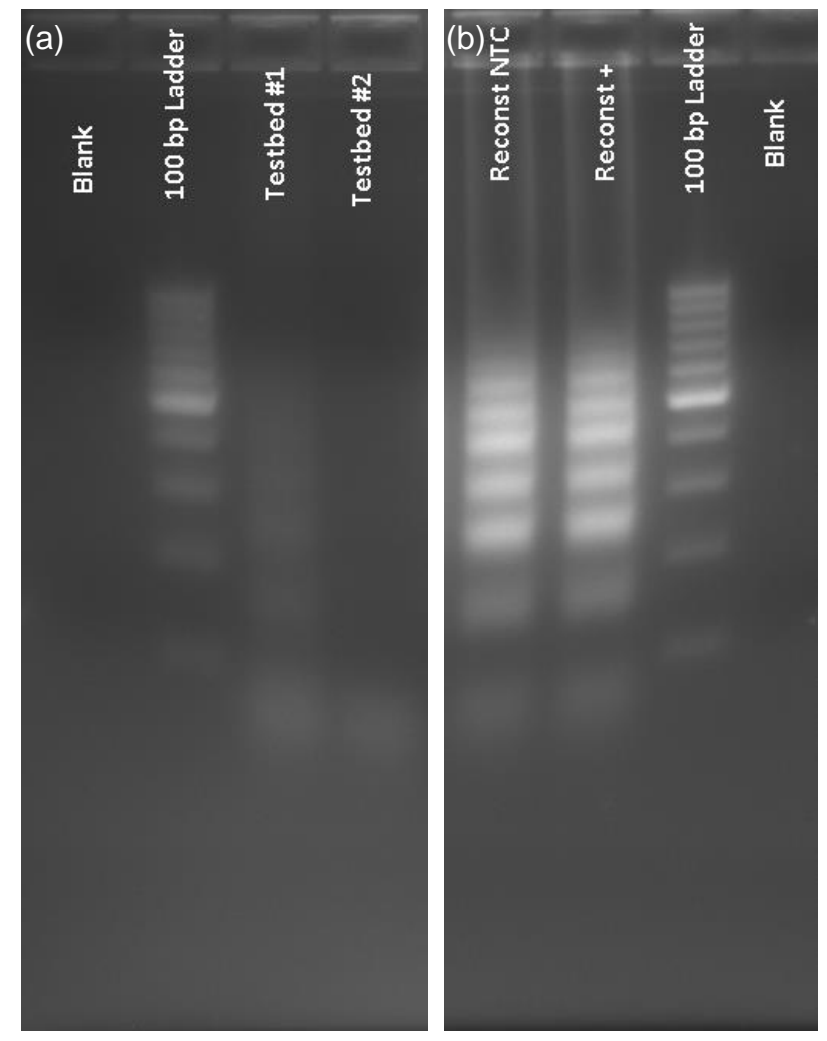

Figure 27: Amplification of reconstituted master-mix materials. (a) Amplification results from two samples reconstituted in the fluidics testbed, with M.tb genomic DNA spiked 
into the reconstitution buffer. Testbed \#1 shows amplification while Testbed \#2 does not. (b) Amplification results from samples reconstituted in standard tubes and amplified on a bench-top thermocycler. The reconstituted NTC sample did not contain M.tb genomic DNA but does show amplification, likely due to amplicon contamination of lab areas.

\section{Conclusions}

We developed a novel reagent storage and reconstitution module to serve as the interface between the sample preparation subunit and the amplification and detection subunit in our fully-integrated disposable cartridge. This module will replace the original conical reconstitution chamber design from our previous amplification and detection cartridge (Chapter 4).

We have designed the new reconstitution chamber to be a shallow ellipse shape, to maximize passive reagent mixing by diffusion. This chamber was designed to interface directly the polypropylene reaction insert, and we were able to demonstrate successful fluid handling in this module. We have demonstrated the ability to dry LAMP master-mix reagents onto microfiber material, and the initial amplification results of samples reconstituted in the fluidic testbed are promising. We are continuing to optimize the drying process, to improve reconstitution amplification kinetics and to reduce false positive amplification of negative samples. Our goal is to demonstrate reconstitution of the dried master-mix material by the sample preparation eluate, followed by amplification and detection in the fully-integrated cartridge. 


\section{APPENDIX}

\section{APPENDIX A: MANUSCRIPT SUBMISSIONS}

At the time of submission of this thesis, most of the content of Chapter 2 was submitted as a manuscript to the journal PLOS One. KGI has also filed a patent application covering the design of this cartridge (System and Method for Efficient Nucleic Acid Testing, US patent application number 13/860,453 filed April 10, 2013). ${ }^{98}$

The work presented in Chapter 3 was submitted as a manuscript to the journal Sensors and Actuators, A. Keck Graduate Institute has filed a provisional patent application covering the design of this valve (Check Valve, US provisional patent application number 61/621,989 filed April 9, 2012). ${ }^{99}$

The work presented in Chapter 4 was published as a paper in the SPIE Conference Proceedings. ${ }^{62}$ 


\section{APPENDIX B: TEAM ORGANIZATION}

The work presented in this thesis is part of a highly collaborative team effort involving other scientists and engineers at Keck Graduate Institute as well as external collaborators. Included below is an organization chart, illustrating the primary personnel in the key subsystems that make up the project as a whole. My specific contributions are included as part of the list of the KGI teams working on each sub-project.

\section{Principal Investigator: Angelika \\ Niemz}

Goal: Develop a fully-integrated NAAT device to diagnose infectious diseases, such as TB, at the point of care in low-resource settings

\begin{tabular}{|c|c|c|c|}
\hline \multicolumn{2}{|c|}{$\begin{array}{l}\text { Assay Development: } \\
\mathrm{KGI} \text {, external collaborators }\end{array}$} & \multicolumn{2}{|c|}{$\begin{array}{l}\text { Device Development: } \\
\mathrm{KGI} \text {, external collaborators }\end{array}$} \\
\hline Sample Preparation & $\begin{array}{l}\text { Lateral Flow } \\
\text { Detection }\end{array}$ & $\begin{array}{l}\text { Sample Prep } \\
\text { Module }\end{array}$ & $\begin{array}{l}\text { Amplification and } \\
\text { Detection Module }\end{array}$ \\
\hline $\begin{array}{l}\text { University of } \\
\text { Washington } \\
\text { Seattle Biomed } \\
\text { Claremont BioSolutions } \\
\text { PATH } \\
\text { Seattle King County } \\
\text { KGI }\end{array}$ & $\begin{array}{l}\text { Claremont BioSolutions } \\
\text { KGI: Kristina Roskos }\end{array}$ & Claremont BioSolutions & $\begin{array}{l}\text { KGI: Anna Hickerson } \\
\text { Kristina Roskos } \\
\text { Hsiang-Wei Lu }\end{array}$ \\
\hline \multicolumn{2}{|c|}{$\begin{array}{l}\text { Nucleic Acid } \\
\text { Amplification }\end{array}$} & \multicolumn{2}{|c|}{ Integrated System } \\
\hline \multicolumn{2}{|c|}{$\begin{array}{l}\text { KGI: Supriya Kadam } \\
\text { Eric Houghton } \\
\text { Kristina Roskos }\end{array}$} & \multicolumn{2}{|c|}{$\begin{array}{l}\text { Claremont BioSolutions } \\
\text { KGI: Anna Hickerson } \\
\text { Kristina Roskos } \\
\text { Hsiang-Wei Lu }\end{array}$} \\
\hline
\end{tabular}




\section{APPENDIX C: PRODUCT REQUIREMENT MATRIX}

At the beginning of this project, the team developed extensive product requirements with maximum and minimum acceptance criteria. After the project had begun, the conference entitled Diagnostics Innovation in China: Bring Chinese TB and HIV Molecular Diagnostics to Market (Shanghai, September 25-26, 2012) distributed a target product profile (TPP), detailing the expectations for TB diagnostics in development. A group of Master of BioScience (MBS) students, along with conversations with our lab team, compared our team's product requirements document with that of the TPP, resulting in the product requirement matrix as included on the next page. Furthermore, they added columns to demonstrate how these requirements are being met by our current, simple system, how they will be met with our future, expanded system, and defined the benchmark for performance. 


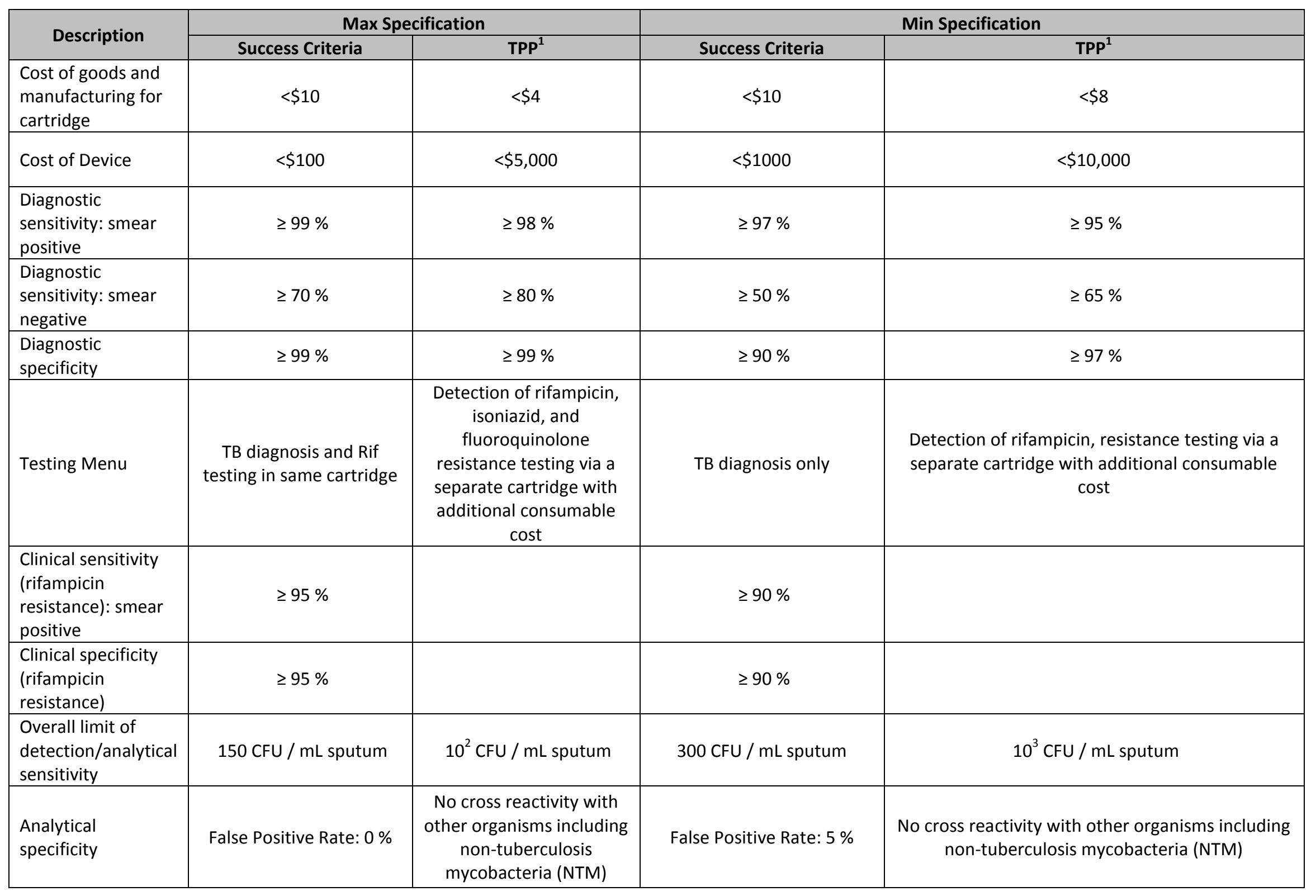




\begin{tabular}{|c|c|c|c|c|}
\hline \multirow{2}{*}{ Description } & \multicolumn{2}{|c|}{ Max Specification } & \multicolumn{2}{|r|}{ Min Specification } \\
\hline & Success Criteria & TPP $^{1}$ & Success Criteria & TPP $^{1}$ \\
\hline Reagent kit stability & $\begin{array}{l}\text { Stable for } \geq 12 \text { month at } \\
\text { RT, for } 1 \text { month at } 45^{\circ}, \\
\text { failure rate }<5 \% \text { at } \\
\text { expiration, and } \\
\text { withstands transport at } \\
\leq 0^{\circ} \mathrm{C}\end{array}$ & $\begin{array}{l}24 \text { months at } 40^{\circ} \mathrm{C}, 70 \% \\
\text { humidity, includes } \\
\text { transport stress ( } 48 \text { hours } \\
\text { at } 50^{\circ} \mathrm{C} \text { ) }\end{array}$ & $\begin{array}{c}\text { Stable for } \geq 12 \text { month at } 4^{\circ} \text {, } \\
\text { for } 1 \text { month at } 25^{\circ} \text {, failure } \\
\text { rate }<10 \% \text { at expiration, } \\
\text { and withstands transport at } \\
\leq 0^{\circ} \mathrm{C}\end{array}$ & $\begin{array}{c}12 \text { months at } 30^{\circ} \mathrm{C}, 70 \% \text { humidity, includes } \\
\text { transport stress }\left(48 \text { hours at } 50^{\circ} \mathrm{C}\right)\end{array}$ \\
\hline $\begin{array}{l}\text { Thermal tolerance } \\
\text { of platform/assay }\end{array}$ & N/A & $\begin{array}{l}\text { Operation between } 15^{\circ} \mathrm{C} \\
\text { and } 40^{\circ} \mathrm{C}\end{array}$ & N/A & Operation between $15^{\circ} \mathrm{C}$ and $35^{\circ} \mathrm{C}$ \\
\hline Reagent Integration & $\begin{array}{c}\text { All reagents on board, } \\
\text { possibly one external } \\
\text { liquefying reagent }\end{array}$ & $\begin{array}{l}\text { All reagents in } \\
\text { consumable }\end{array}$ & $\begin{array}{l}\text { Reagent addition to } \\
\text { cartridge for alpha } \\
\text { prototype }\end{array}$ & $<4$ external reagents \\
\hline $\begin{array}{l}\text { Sample preparation } \\
\text { and assay } \\
\text { processing }\end{array}$ & $\begin{array}{c}3 \text { steps: (1) sample intro, } \\
\text { (2) push go, (3) record } \\
\text { result }\end{array}$ & Integrated & $\begin{array}{l}\text { one manual step after } \\
\text { initiation, (step 2) upon } \\
\text { timer sounding }\end{array}$ & $\begin{array}{l}\text { Minimal sample processing; no more than 3-5 } \\
\text { steps (requiring operator intervention) }\end{array}$ \\
\hline User interface & $\begin{array}{l}\text { Intuitive user interface, } \\
\text { minimum \# of buttons } \\
\text { right on device - } \\
\text { microprocessor }\end{array}$ & $\begin{array}{c}\text { Simple test menu, } \\
\text { integrated LCD screen; } \\
\text { simple key pad or touch } \\
\text { screen }\end{array}$ & $\begin{array}{l}\text { Intuitive user interface on } \\
\text { external laptop connected } \\
\text { to device - external control }\end{array}$ & $\begin{array}{l}\text { Simple test menu, integrated LCD screen; simple } \\
\text { key pad or touch screen }\end{array}$ \\
\hline $\begin{array}{l}\text { Electronics and } \\
\text { data analysis }\end{array}$ & $\begin{array}{l}\text { Entire unit is a handheld } \\
\text { device (requires } \\
\text { microprocessor) }\end{array}$ & Integrated & $\begin{array}{c}\text { Alpha prototype unit is a } \\
\text { handheld device, with small } \\
\text { external computer }\end{array}$ & Separate computer required \\
\hline Portability & $\begin{array}{l}\text { Handheld device }(<0.9 \\
\mathrm{kg}) \text { (requires } \\
\text { microprocessor); } 1+\mathrm{w}+\mathrm{h}= \\
25 \mathrm{~cm}\end{array}$ & $\begin{array}{l}\text { Small, portable device }(<2 \\
\mathrm{kg}) \text { or handheld analyzer }\end{array}$ & $\begin{array}{c}\text { Alpha prototype unit is a } \\
\text { handheld device }(<2.7 \mathrm{~kg}) \text {, } \\
\text { with small external } \\
\text { computer, } 1+\mathrm{w}+\mathrm{h}=50 \mathrm{~cm}\end{array}$ & $\begin{array}{c}\text { Small, table-top analyzer }(<5 \mathrm{~kg}) \text { or portable } \\
\text { device }\end{array}$ \\
\hline Cold Chain & $\begin{array}{c}\text { No refrigeration needed } \\
\text { for cartridge, device } \\
\text { withstands transport at } \\
\leq 0^{\circ} \mathrm{C}\end{array}$ & $\begin{array}{l}\text { None required at any } \\
\text { point in supply chain or } \\
\text { storage }\end{array}$ & $\begin{array}{l}\text { For alpha prototype some } \\
\text { reagents require } \\
\text { refrigeration, with path } \\
\text { towards stable formulation }\end{array}$ & $\begin{array}{c}\text { None required at any point in supply chain or } \\
\text { storage }\end{array}$ \\
\hline Time - to - result & $\begin{array}{l}\leq 5 \text { min hands-on time } \\
\text { concentrated at the front } \\
\text { end; results in } \leq 40 \mathrm{~min} \\
\text { from sample introduction }\end{array}$ & $<1 \mathrm{~h}$ & $\begin{array}{c}\leq 15 \text { min hands-on time } \\
\text { concentrated at the front } \\
\text { end, results in } \leq 1 \mathrm{~h} \text { from } \\
\text { sample introduction }\end{array}$ & $<2 \mathrm{~h}$ \\
\hline Throughput & - & $\begin{array}{l}>48 \text { samples per day, } \\
\text { asynchronously }\end{array}$ & - & 12 tests per 8 -hour day \\
\hline
\end{tabular}




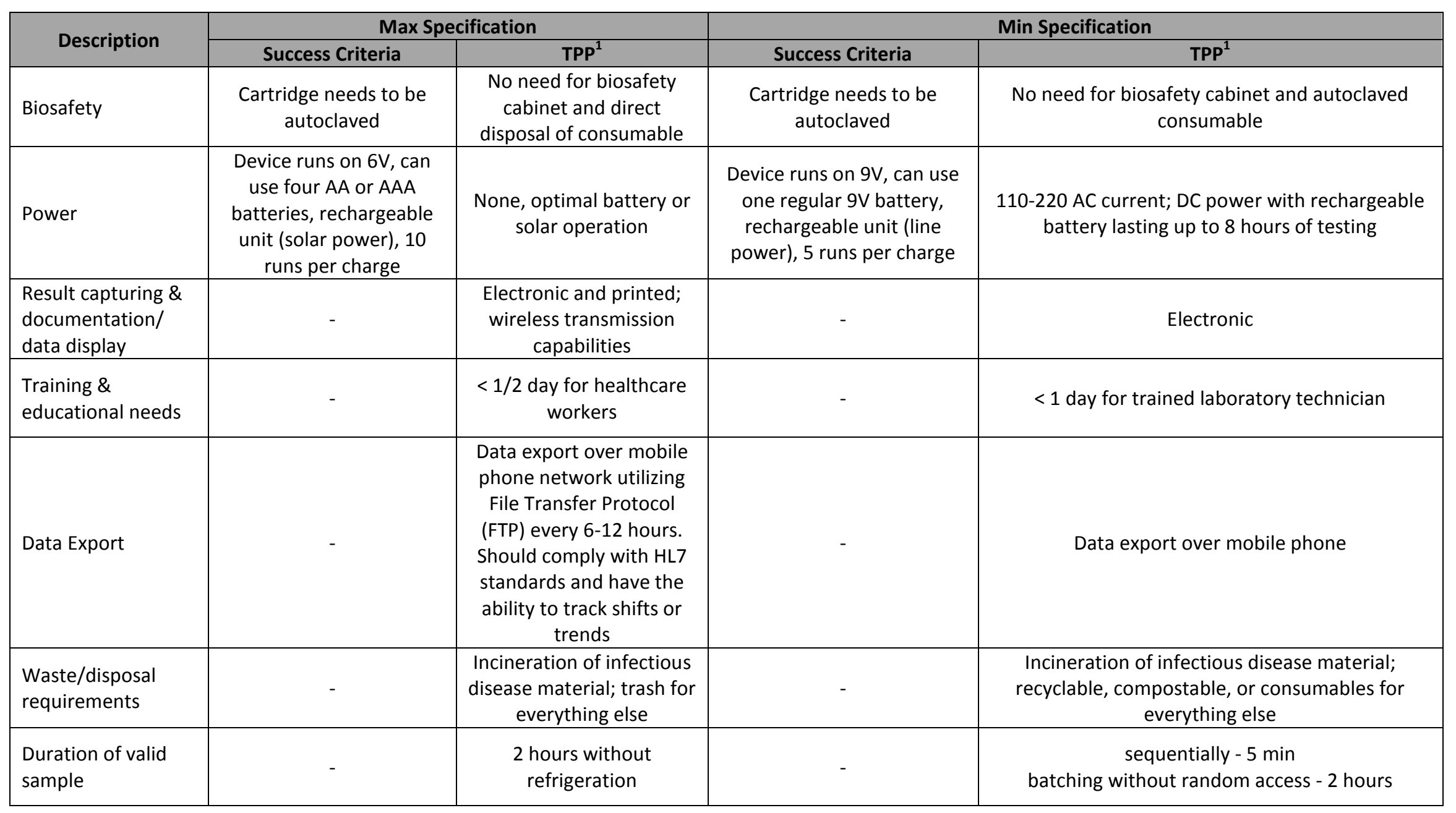




\begin{tabular}{|c|c|c|c|}
\hline Description & Final version 1 (simple system) & Final version 2 (expanded system) & Benchmark \\
\hline Cost of Device & $\$ 170$, plus $\$ 30$ markup (18\%) & $\begin{array}{l}<\$ 5000 \text { for four module unit with LF } \\
\text { reader, connectivity }\end{array}$ & $\begin{array}{l}\text { Cepheid GeneXpert: } \$ 17 \mathrm{k} \text { per } 4 \text { module instrument } \\
\text { ExWorks, which boils down to COGM of } \$ 13 \mathrm{k}^{2}\end{array}$ \\
\hline $\begin{array}{l}\text { Diagnostic sensitivity: } \\
\text { smear positive }\end{array}$ & $\geq 95 \%$ & $\geq 95 \%$ & Cepheid GeneXpert: $98.3 \% \%^{3,4}$ \\
\hline $\begin{array}{l}\text { Diagnostic sensitivity: } \\
\text { smear negative }\end{array}$ & $\geq 65 \%$ & $\geq 65 \%$ & Cepheid GeneXpert: $76.9 \%^{3,4}$ \\
\hline Diagnostic specificity & $\geq 97 \%$ & $\geq 97 \%$ & Cepheid GeneXpert: 99.0\% ${ }^{3,4}$ \\
\hline Testing Menu & TB diagnosis only & $\begin{array}{l}\text { Detection of rifampicin, resistance } \\
\text { testing via a separate cartridge with } \\
\text { additional consumable cost }\end{array}$ & \\
\hline $\begin{array}{l}\text { Clinical sensitivity } \\
\text { (rifampicin resistance): } \\
\text { smear positive }\end{array}$ & $\mathrm{N} / \mathrm{A}$ & $\geq 95 \%$ & $\begin{array}{l}\text { Cepheid GeneXpert: } 94.1 \%^{3,4} \\
\quad \text { Line Probe: } 93-98.9 \%^{5,6}\end{array}$ \\
\hline $\begin{array}{l}\text { Clinical specificity } \\
\text { (rifampicin resistance) }\end{array}$ & N/A & $\geq 97 \%$ & $\begin{array}{l}\text { Cepheid GeneXpert: } 97 \%^{3,4} \\
\text { Line Probe: } 99.4-100.0 \% \%^{5,6}\end{array}$ \\
\hline $\begin{array}{l}\text { Overall limit of } \\
\text { detection/analytical } \\
\text { sensitivity }\end{array}$ & $\leq 10^{3} \mathrm{CFU} / \mathrm{mL}$ sputum & $\leq 10^{3} \mathrm{CFU} / \mathrm{mL}$ sputum & $\begin{array}{c}\text { Cepheid GeneXpert: overall LOD } 131 \mathrm{CFU} / \mathrm{mL} \text {, assay } \\
\text { LOD } 4.5 \text { genomes } / \mathrm{rxn}^{8}\end{array}$ \\
\hline Analytical specificity & $\begin{array}{l}\text { No cross reactivity with other organisms } \\
\text { including non-tuberculosis mycobacteria } \\
\text { (NTM) }\end{array}$ & $\begin{array}{l}\text { No cross reactivity with other } \\
\text { organisms including non-tuberculosis } \\
\text { mycobacteria (NTM) }\end{array}$ & \\
\hline Reagent kit stability & $\begin{array}{l}\text { Stable for } \geq 12 \text { month at } 4^{\circ} \text {, for } 1 \text { month at } \\
25^{\circ} \text {, failure rate }<10 \% \text { at expiration, and } \\
\text { withstands transport at } \leq 0^{\circ} \mathrm{C}\end{array}$ & $\begin{array}{l}12 \text { months at } 30^{\circ} \mathrm{C}, 70 \% \text { humidity, } \\
\text { includes transport stress (48 hours at } \\
50^{\circ} \mathrm{C} \text { ) }\end{array}$ & $\begin{array}{c}\text { The Xpert MTB/RIF cartridges and reagents should } \\
\text { be stored at } 2-28^{\circ} \mathrm{C}^{8}\end{array}$ \\
\hline $\begin{array}{l}\text { Thermal tolerance of } \\
\text { platform/assay }\end{array}$ & Operation between $15^{\circ} \mathrm{C}$ and $40^{\circ} \mathrm{C}$ & Operation between $15^{\circ} \mathrm{C}$ and $40^{\circ} \mathrm{C}$ & $\begin{array}{l}\text { Cepheid GeneXpert: operation between } 2^{\circ} \mathrm{C} \text { and } \\
\qquad 30^{\circ} \mathrm{C}^{3}\end{array}$ \\
\hline
\end{tabular}




\begin{tabular}{|c|c|c|c|}
\hline Description & Final version 1 (simple system) & Final version 2 (expanded system) & Benchmark \\
\hline Reagent Integration & $\begin{array}{l}\text { wash, elution buffer, master-mix, and LF } \\
\text { strips in cartridge, liquefying/disinfecting } \\
\text { reagent and binding buffer part of sample } \\
\text { collection device }\end{array}$ & $\begin{array}{l}\text { wash, elution buffer, master-mix, } \\
\text { and LF strips in cartridge, } \\
\text { liquefying/disinfecting reagent and } \\
\text { binding buffer part of sample } \\
\text { collection device }\end{array}$ & Cepheid GeneXpert: reagent addition to cartridge ${ }^{3}$ \\
\hline $\begin{array}{l}\text { Sample preparation and } \\
\text { assay processing }\end{array}$ & $\begin{array}{c}\text { Sputum produced into cup, release } \\
\text { liquefying / disinfecting reagent, shake, } \\
\text { incubate } 10 \text { min, via syringe add glycine } \\
\text { buffer, aspirate sample, inject into cartridge, } \\
\text { push go }\end{array}$ & $\begin{array}{l}\text { Sputum produced into cup, release } \\
\text { liquefying / disinfecting reagent, } \\
\text { shake, incubate } 10 \text { min, via syringe } \\
\text { add glycine buffer, aspirate sample, } \\
\text { inject into cartridge, push go }\end{array}$ & Cepheid GeneXpert: 3 hands-on steps ${ }^{3}$ \\
\hline User interface & $\begin{array}{l}\text { Simple test menu, integrated LCD screen; } \\
\text { simple key pad or touch screen }\end{array}$ & $\begin{array}{c}\text { Simple test menu, integrated LCD } \\
\text { screen; simple key pad or touch } \\
\text { screen }\end{array}$ & \\
\hline $\begin{array}{l}\text { Electronics and data } \\
\text { analysis }\end{array}$ & Integrated & Integrated & $\begin{array}{c}\text { Cepheid GeneXpert: instrument, computer, and } \\
\text { barcode wand reader }{ }^{8}\end{array}$ \\
\hline Portability & $\begin{array}{c}\text { Small, portable device }(<2 \mathrm{~kg} \text { ) or handheld } \\
\text { analyzer }\end{array}$ & $\begin{array}{l}\text { Small, table-top analyzer }(<5 \mathrm{~kg}) \text { or } \\
\text { portable device }\end{array}$ & Cepheid GeneXpert: table-top, $1+w+h=79 \mathrm{~cm}$ \\
\hline Cold Chain & $\begin{array}{l}\text { For alpha prototype some reagents require } \\
\text { refrigeration, with path towards stable } \\
\text { formulation }\end{array}$ & $\begin{array}{l}\text { None required at any point in supply } \\
\text { chain or storage }\end{array}$ & \\
\hline Time - to - result & $<1 \mathrm{~h}$ & $<1 \mathrm{~h}$ & Cepheid GeneXpert: $<2 \mathrm{~h}^{3}$ \\
\hline Throughput & $\begin{array}{l}6 \text { tests per 8h day, for single instrument run } \\
\text { in series }\end{array}$ & 24 tests per $8 \mathrm{~h}$ day, random access & Cepheid GeneXpert: 1,4 , or 16 samples in parallel \\
\hline Biosafety & $\begin{array}{l}\text { No need for biosafety cabinet and direct } \\
\text { disposal of consumable }\end{array}$ & $\begin{array}{l}\text { No need for biosafety cabinet and } \\
\text { direct disposal of consumable }\end{array}$ & Cepheid GeneXpert: same as smear microscopy ${ }^{3}$ \\
\hline Power & $\begin{array}{c}\text { rechargeable battery lasting up to } 8 \text { hours of } \\
\text { testing }\end{array}$ & $\begin{array}{c}\text { rechargeable battery lasting up to } 8 \\
\text { hours of testing }\end{array}$ & \\
\hline
\end{tabular}
binding buffer part of sample

quefying/disinfecting reagent and buffer part of sample

putum produced into cup, release

liquefying / disinfecting reagent,

shake, incubate $10 \mathrm{~min}$, via syringe Simple test menu, integrated LCD le key pad or touch barcode wand reader ${ }^{8}$ 


\begin{tabular}{|c|c|c|c|}
\hline Description & Final version 1 (simple system) & Final version 2 (expanded system) & Benchmark \\
\hline $\begin{array}{l}\text { Result capturing \& } \\
\text { documentation/ } \\
\text { data display }\end{array}$ & simple visual lateral flow readout & $\begin{array}{l}\text { Electronic - LF reader; wireless } \\
\text { transmission capabilities }\end{array}$ & \\
\hline $\begin{array}{l}\text { Training \& educational } \\
\text { needs }\end{array}$ & $<1 / 2$ day for healthcare workers & $<1 / 2$ day for healthcare workers & $\begin{array}{c}\text { Cepheid GeneXpert: } 2 \text { days for non-experienced lab- } \\
\text { techs }{ }^{3}\end{array}$ \\
\hline \multirow[t]{4}{*}{$\begin{array}{l}\text { Service/ } \\
\text { Maintenance/ } \\
\text { Calibration }\end{array}$} & $\begin{array}{c}\text { No annually scheduled preventative } \\
\text { maintenance required }\end{array}$ & $\begin{array}{c}\text { No annually scheduled preventative } \\
\text { maintenance required }\end{array}$ & $\begin{array}{c}\text { Cepheid GeneXpert: annual calibration. General } \\
\text { warranty is } 12 \text { months }^{7}\end{array}$ \\
\hline & & $\begin{array}{c}\text { device has capability to send an alert } \\
\text { or to be detected remotely when it is } \\
\text { not functioning properly }\end{array}$ & \\
\hline & $\begin{array}{l}\text { Mean time to failure of at least } 12 \text { months; } \\
\qquad 18 \text { months preferred }\end{array}$ & $\begin{array}{l}\text { Mean time to failure of at least } 12 \\
\text { months; } 18 \text { months preferred }\end{array}$ & \\
\hline & No calibration required & No calibration required & \\
\hline Regulatory Requirements & $\begin{array}{l}\text { preliminary document control system, } \\
\text { DHF/DMR, risk analysis, minimal quality }\end{array}$ & $\begin{array}{c}\text { Must be produced according to } \\
\text { cGMPs, ISO } 13485 \text { and authorized by } \\
\text { GHTF }\end{array}$ & \\
\hline Data Export & & $\begin{array}{c}\text { Data export over mobile phone } \\
\text { network utilizing File Transfer } \\
\text { Protocol (FTP) every 6-12 hours. } \\
\text { Should comply with HL7 standards } \\
\text { and have the ability to track shifts or } \\
\text { trends }\end{array}$ & \\
\hline $\begin{array}{l}\text { Waste/disposal } \\
\text { requirements }\end{array}$ & Incineration or trash for everything & Incineration or trash for everything & $\begin{array}{l}\text { Cepheid GeneXpert: additional waste compared to } \\
\text { smear microscopy }{ }^{3}\end{array}$ \\
\hline Duration of valid sample & & 2 hours without refrigeration & $\begin{array}{l}\text { Cepheid GeneXpert: start the test within } 30 \text { min of } \\
\text { adding the sample to the cartridge }{ }^{8}\end{array}$ \\
\hline
\end{tabular}


1،"TPP for Optimal and Minimal Product Characteristics for proposed tuberculosis diagnostic.” Diagnostics Innovation in China: Bring Chinese TB and HIV Molecular Diagnostics to Market. Shanghai, September 25-26, 2012

${ }^{2}$ Cepheid Cares. 2012. Cepheid. November 11, 2012. www.cepheidcares.com/tb/cepheid-vision.html\#pricing

${ }^{3}$ Boehme, Catharina. "Value chain in action: Xpert MTB/RIF." FIND. Montreal. July 9, 2012

${ }^{4}$ Boehme, Catharina et al. Rapid Molecular Detection of Tuberculosis and Rifampin Resistance. NEJM 2010, 363, 1005

${ }^{5}$ Huyen, Mai NT et al. Validation of the GenoType MTBDRplus assay for diagnosis of multidrug resistant tuberculosis in South Vietnam. BMC Infectious Diseases 2010, 10, 149

${ }^{6}$ Barnard, Marinus et al. Rapid Molecular Screening for Multidrug-Resistant Tuberculosis in a High-Volume Public Health Laboratory in South Africa. Am. J.Res.Crit.Care 2008, 177, 787

${ }^{7}$ Helb, Danica et al. Rapid Detection of Mycobacterium tuberculosis and Rifampin Resistance by Use of On-Demand, Near-Patient Technology. J.Clin Microbiol. 2010, 48, 22

"Xpert® MTB/RIF: Two-hour detection of MTB and resistance to rifampicin.” Cepheid product brochure. $300-7810$ Rev. A. April 2009 


\section{APPENDIX D: CARTRIDGE MANUFACTURING STANDARD OPERATING PROCEDURE}

To ensure quality and reproducibility of cartridge assembly, I compiled the following standard operating procedure (SOP). This SOP is considered to be a working document, stored in the shared lab resource folder with all team members having access. The SOP is

included in its entirety below. The Niemz Lab is developing SOPs for all components and processes to assure quality. 
Standard Operating Procedure: Amplification and Detection Cartridge

DESCRIPTION: Assembly protocol for manufacturing Amplification and Detection Cartridge version 1 for either EXPAR-LF or LAMP-LF. PURPOSE:

This SOP details the assembly protocol for manufacturing cartridges.

\section{MATERIALS}

\begin{tabular}{|c|l|l|l|l|l|l|}
\hline $\begin{array}{l}\text { Item } \\
\text { No. }\end{array}$ & Source & Part No. & Item Description & Unit Qty. & $\begin{array}{l}\text { Lot No/ } \\
\text { Prep date }\end{array}$ & $\begin{array}{l}\text { Quantity } \\
\text { Used }\end{array}$ \\
\hline 1 & Lee Co. & $\begin{array}{l}\text { CCPI2510 } \\
\text { 014S D }\end{array}$ & Check valve, 2.5mm & & 2 \\
\hline 2 & Qosina & 49005 & Injection Port with Al Cap & & & 2 \\
\hline 3 & Qosina & 11628 & 8 " x 10" Autoclave Bag & & & $\begin{array}{l}2 \times 1 / 8 ” \\
\text { slice }\end{array}$ \\
\hline 4 & $\begin{array}{l}\text { McMaster- } \\
\text { Carr }\end{array}$ & & Silicone rubber cord & & & 2 sq.in \\
\hline 5 & $\begin{array}{l}\text { McMaster- } \\
\text { Carr }\end{array}$ & & Silicone Foam & & & 1 \\
\hline 6 & KGI & & Injection molded PP top card & & & 1 \\
\hline 7 & KGI & & Injection molded PP bottom card & & & 2 \\
\hline 8 & KGI & & Dry reagent LF strips & & & $\sim 3$ mL \\
\hline 9 & KGI & & $1 \mathrm{M}$ Na2SO4 electrolyte solution & & & 4 \\
\hline 10 & & & $18 G$ needle tip (as electrode) & & & 2 \\
\hline 11 & & & $21.5 G$ needle tip & & & 2 \\
\hline 12 & & & 1 mL syringe & & & 1 \\
\hline 13 & & & 3 mL syringe & & & \\
\hline
\end{tabular}




\section{A. Off-the-shelf components}

\section{Lee Company Check Valves}

a) Prepare valves upon receipt by autoclaving in glass beaker (covered with foil) for 50 minutes followed by 15 minutes drying.

b) Once autoclaved, valves should be stored in a clean, dry Ziploc bag inside the laminar flow hood in the clean assembly lab.

c) Use tweezers to handle valves in clean assembly room.

\section{Qosina Injection Ports}

a) Upon receipt, Qosina box should be opened in hallway outside of clean assembly lab. Only inner bag containing injection ports should be brought inside clean assembly lab.

b) Store sealed bag in cabinet under laminar flow hood work area in clean assembly lab.

c) Use clean, gloved hands to handle injection ports.

\section{Qosina Autoclave Bags}

a) Upon receipt, Qosina box should be opened in hallway outside of clean assembly lab. Only inner bag containing autoclave bags should be brought inside clean assembly lab.

b) Store sealed bag in cabinet under laminar flow hood work area in clean assembly lab.

c) Use clean, gloved hands to handle autoclave bags.

\section{McMaster-Carr Silicone Rubber Cord}

a) Prepare septa by cutting cord into $1 / 16$ " thick slices upon receipt.

b) Decontaminate septa by autoclaving in glass beaker (covered with foil) for 50 minutes followed by 15 minutes drying.

c) Once autoclaved, valves should be stored in a clean, dry Ziploc bag inside the laminar flow hood in the clean assembly lab.

d) Use tweezers to handle septa in clean assembly room.

\section{McMaster-Carr Silicone Foam}

a) Upon receipt, Qosina box should be opened in hallway outside of clean assembly lab. Foam may or may not be packaged in another inner bag. Only foam should be brought inside the clean assembly lab.

b) Store foam in drawer under laminar flow hood work area in clean assembly lab.

c) Use clean, gloved hands to handle autoclave bags.

\section{Additional Tools for Assembly}

a) When possible, all tools used for assembly should be autoclaved for 50 minutes followed by drying for 15 minutes prior to being brought into the clean assembly room.

b) If autoclaving is not possible, tools should be decontaminated with $10 \%$

bleach solution prior to being brought into the clean assembly room.

\section{B. Raw materials for manufacturing components in-house}

\section{Polypropylene Pellets for Injection Molding}


a) Upon receipt, store box containing pellets under injection molding bench, keeping pellets as cool and dry as possible.

b) While injection molding, use smaller box on bench to refill Morgan Press barrel.

\section{Dry Reagent Lateral Flow Strips}

a) Upon receipt, store in clean assembly room.

\section{METHODOLOGY}

\section{A. Preparing Injection molded polypropylene cartridge halves}

1. Drilling top (lateral flow) half - in Prototype Lab

d) Drill holes through card for the pump chambers (2) using 0.213 " drill bit.

e) Drill holes through card for the reaction pouch inlets/outlets (4 total) using 0.067 " drill bit.

f) Trim fly from all holes such that there are no sharp points protruding from the flat surface of the PP card.

g) Trim residual PP splay (result of injection molding pieces, may vary in amount) from corners of card to ensure card fits into heater plate area.

2. Drilling bottom (reaction/pump) half - in Prototype Lab

a) Drill holes through card for the pump chambers (2) using 0.128 " drill bit.

b) Drill holes through card for the reaction pouch inlets/outlets (4 total) using 0.067 " drill bit.

c) Trim fly from all holes such that there are no sharp points protruding from the flat surface of the PP card.

d) Trim residual PP splay (result of injection molding pieces, may vary in amount) from corners of card to ensure card fits into heater plate area.

3. Autoclave - in Autoclave Room / Clean Assembly Lab

a) Once polypropylene pieces are drilled, place in dedicated autoclave bin and cover with foil.

b) Dry-autoclave for 50 minutes followed by 15 minutes drying.

c) After autoclaving, bring bin directly into clean assembly lab and store in Ziploc bag until use.

\section{B. Heat-Sealing pouches}

1. Bottom half - in Clean Assembly Lab

a) Cut polypropylene autoclave bags into 1.5 " strips, and then cut strips into thirds and separate making PP film pieces.

b) Using one PP film piece, seal reaction pouches onto one polypropylene injection molded/drilled bottom half using heated arbor press set to $350^{\circ} \mathrm{F}$ with reaction pouch version 1 stamp attached.

c) Trim excess PP film around reaction pouch seams using X-acto knife.

d) Using one PP film piece, seal pump pouches onto polypropylene bottom half using heated arbor press with pump pouch version 1 stamp attached (and previous heat setting).

e) Trim excess PP film around pump pouch seams.

2. Top half - in Clean Assembly Lab 
a) Place to one $4 \mathrm{~mm}$ lateral flow strip into each lateral flow strip groove in one polypropylene injection molded/drilled top half, in anti-parallel orientation with dry reagent conjugate pads over reaction pouch outlet tubing.

b) Using one PP film piece, seal lateral flow pouches onto polypropylene top half using heated arbor press with lateral flow pouch version 1 stamp attached (and previous heat setting).

c) Trim excess PP film around lateral flow pouch seams.

\section{Valve and septa components}

1. Prepare components - in Autoclave Room / Clean Assembly Room

a) Place components into a glass beaker and into dedicated autoclave bin. Cover with foil.

b) Dry-autoclave for 50 minutes followed by 15 minutes drying.

c) After autoclaving, bring bin and beaker directly into clean assembly lab and store in Ziploc bag until use.

2. Insert Valves - in Clean Assembly Lab

a) Start with one bottom PP card that has reaction and pump pouches already stamped.

b) Use tweezers to insert one valve in each of the outlet tubings (two total per PP card), with larger, ribbed side of valve on the reaction pouch side of the tubing.

c) Use hex key / allen wrench to press-fit valves into place.

3. Insert Septa - in Clean Assembly Lab

a) Start with one top PP card that has lateral flow strip pouches already stamped.

b) Use tweezers to insert one septum in each of the inlet tubings (two total per PP card).

c) Use hex key / allen wrench to press-fit septa into place.

\section{Joining polypropylene halves - in Clean Assembly Room}

1. Plug in hot glue gun and have one top and one bottom PP card ready for assembly (pouches stamped and trimmed, with valves/septa/ LF strips already inserted).

2. Once glue gun is ready, deposit glue around inner faces of all fluid conduits (reaction and pump pouch tubings and bosses).

3. Press fit top and bottom PP cards together.

4. Deposit glue around outer faces of all fluid conduits.

\section{E. Insulating foam - in Clean Assembly Room}

1. Cut large foam sheet to size for cartridges $-2 \frac{1 / 4}{4} \times 1 \frac{1}{2}$ "

2. Cut each smaller piece into four parts.

3. Keep pieces with cartridge, to be assembled inside cartridge after electrolytic chambers have been filled and cartridge is ready to be placed on heater unit.

\section{F. Adding and filling electrolyte chambers - in Clean Assembly Room}

1. Take two Qosina injection ports per cartridge.

2. Insert on port into each pump chamber fluid conduit and press fit until chamber cannot turn in place.

\section{G. Individually bag each cartridge}

1. Place on assembled cartridge and one set of four foam pieces into a small Ziploc bag.

2. Each cartridge will remain individually bagged until taken out just before running on heater unit. 


\section{APPENDIX E: FAILURE MODE AND EFFECTS ANALYSIS}

A group of MBS students, along with conversations with our lab team, prepared a Failure Mode and Effects Analysis (FMEA) to consider how individual sub-components of this overall project can fail. For each failure mode, the severity $(\mathrm{S})$, occurrence $(\mathrm{O})$, and detection level (D) were assessed, along with controls that are currently being implemented to mitigate risk. Lastly, a risk priority number (RPN) was assigned to indicate failure modes, and ultimately system sub-components, which carry the highest risk.

I participated in the FMEA analysis related to the cartridges, including amplification and detection cartridge components, reagent storage and the amplification and detection assays as performed on the cartridge. The FMEA document is included below in its entirety. 


\begin{tabular}{|c|c|c|c|c|c|c|c|c|c|c|c|c|c|c|}
\hline \multicolumn{10}{|c|}{ Risk Estimation and Evaluation } & \multicolumn{5}{|c|}{ Risk Control } \\
\hline & Function & $\begin{array}{l}\text { Failure } \\
\text { Mode }\end{array}$ & Failure Effect & $\mathbf{S}$ & $\begin{array}{l}\text { Cause of } \\
\text { Failure } \\
\text { Mode }\end{array}$ & $\mathbf{O}$ & $\begin{array}{l}\text { Current } \\
\text { Controls }\end{array}$ & $\mathbf{D}$ & RPN & $\begin{array}{l}\text { Mitigation } \\
\text { Strategy }\end{array}$ & $\mathbf{S}^{*}$ & O* & $\mathbf{D}^{*}$ & RPN* \\
\hline \multirow{5}{*}{ OmniValve } & \multirow{5}{*}{$\begin{array}{l}\text { Direct liquids into } \\
\text { appropriate } \\
\text { chambers using a } \\
\text { turning } \\
\text { mechanism to } \\
\text { execute duty }\end{array}$} & \multirow{2}{*}{$\begin{array}{l}\text { Valve } \\
\text { turned to } \\
\text { incorrect } \\
\text { angle }\end{array}$} & $\begin{array}{l}\text { Sample cannot be } \\
\text { introduced into } \\
\text { cartridge, cartridge } \\
\text { will not function }\end{array}$ & 4 & $\begin{array}{l}\text { Stepper motor } \\
\text { miss-alignment }\end{array}$ & 6 & $\begin{array}{l}\text { valve } \\
\text { homing } \\
\text { step, but no } \\
\text { feedback }\end{array}$ & 2 & 48 & Valve position check & 4 & 3 & 2 & 24 \\
\hline & & & $\begin{array}{l}\text { Sample introduced } \\
\text { into incorrect } \\
\text { cartridge }\end{array}$ & 6 & $\begin{array}{l}\text { Stepper motor } \\
\text { miss-alignment }\end{array}$ & 3 & $\begin{array}{l}\text { Valve } \\
\text { homing } \\
\text { step, but no } \\
\text { feedback }\end{array}$ & 2 & 36 & Valve position check & 6 & 2 & 2 & 24 \\
\hline & & $\begin{array}{l}\text { Valve } \\
\text { clogged }\end{array}$ & $\begin{array}{l}\text { Sample cannot be } \\
\text { introduced into } \\
\text { cartridge, cartridge } \\
\text { will not function }\end{array}$ & 4 & $\begin{array}{l}\text { Thick sputum } \\
\text { mixes with } \\
\text { beads to form a } \\
\text { paste }\end{array}$ & 4 & $\begin{array}{l}\text { Keep motor } \\
\text { running to } \\
\text { ensure no } \\
\text { paste settles } \\
\end{array}$ & 2 & 32 & $\begin{array}{l}\text { Add excess TSP to } \\
\text { further liquefy sample }\end{array}$ & 4 & 2 & 2 & 16 \\
\hline & & \multirow{2}{*}{ Valve stuck } & $\begin{array}{l}\text { Sample cannot be } \\
\text { introduced into } \\
\text { cartridge, cartridge } \\
\text { will not function }\end{array}$ & 4 & $\begin{array}{l}\text { Periods of } \\
\text { inactivity } \\
\text { causes sticking }\end{array}$ & 2 & $\begin{array}{l}\text { Manually } \\
\text { turn valve } \\
\text { prior to } \\
\text { sample } \\
\text { injection }\end{array}$ & 2 & 16 & $\begin{array}{l}\text { Use lubricant to prime } \\
\text { the valve }\end{array}$ & 4 & 1 & 2 & 8 \\
\hline & & & $\begin{array}{l}\text { Overheat and burn } \\
\text { up motor, cartridge } \\
\text { will not function }\end{array}$ & 6 & $\begin{array}{l}\text { Periods of } \\
\text { inactivity } \\
\text { causes sticking }\end{array}$ & 2 & $\begin{array}{l}\text { Manually } \\
\text { turn valve } \\
\text { prior to } \\
\text { sample } \\
\text { injection }\end{array}$ & 2 & 24 & $\begin{array}{l}\text { Use lubricant to prime } \\
\text { the valve }\end{array}$ & 6 & 1 & 2 & 12 \\
\hline
\end{tabular}




\begin{tabular}{|c|c|c|c|c|c|c|c|c|c|c|c|c|c|c|}
\hline \multicolumn{10}{|c|}{ Risk Estimation and Evaluation } & \multicolumn{5}{|c|}{ Risk Control } \\
\hline 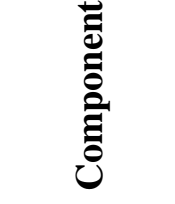 & Function & Failure Mode & Failure Effect & $\mathbf{S}$ & $\begin{array}{l}\text { Cause of } \\
\text { Failure } \\
\text { Mode }\end{array}$ & O & $\begin{array}{l}\text { Current } \\
\text { Controls }\end{array}$ & D & RPN & $\begin{array}{l}\text { Mitigation } \\
\text { Strategy }\end{array}$ & $\mathbf{S}^{*}$ & O* & $\mathbf{D}^{*}$ & RPN* \\
\hline OmniValve & $\begin{array}{l}\text { Separation of } \\
\text { liquid and dry } \\
\text { reagents while } \\
\text { stored }\end{array}$ & $\begin{array}{l}\text { Dry reagents } \\
\text { exposed to } \\
\text { moisture }\end{array}$ & $\begin{array}{l}\text { Reagents and LF } \\
\text { strips inactive }\end{array}$ & 6 & $\begin{array}{l}\text { Valve not } \\
\text { perfectly } \\
\text { sealed }\end{array}$ & 5 & none & 5 & 150 & $\begin{array}{l}\text { Implement seal on } \\
\text { OmniValve }\end{array}$ & 6 & 3 & 5 & 90 \\
\hline \multirow{3}{*}{$\begin{array}{c}\text { Reagent } \\
\text { paper pad }\end{array}$} & \multirow{3}{*}{$\begin{array}{l}\text { Contain reagents } \\
\text { in stable form }\end{array}$} & $\begin{array}{l}\text { Inhomogeneous } \\
\text { master-mix }\end{array}$ & $\begin{array}{l}\text { Potential false } \\
\text { negative }\end{array}$ & 9 & $\begin{array}{l}\begin{array}{l}\text { Reagents not } \\
\text { uniformly } \\
\text { released }\end{array} \\
\text { Amplification } \\
\text { kinetics } \\
\text { inconsistent }\end{array}$ & 5 & None & 10 & 450 & $\begin{array}{l}\text { Internal } \\
\text { Amplification } \\
\text { Control } \\
\text { Monitor release of } \\
\text { reagents }\end{array}$ & 9 & 5 & 3 & 135 \\
\hline & & $\begin{array}{l}\text { Master-mix } \\
\text { reagents not } \\
\text { released }\end{array}$ & False negative & 9 & $\begin{array}{l}\text { No reagents, } \\
\text { no } \\
\text { amplification }\end{array}$ & 4 & None & 10 & 360 & $\begin{array}{l}\text { Internal } \\
\text { Amplification } \\
\text { Control, Detect } \\
\text { release of reagents }\end{array}$ & 9 & 4 & 3 & 108 \\
\hline & & $\begin{array}{l}\text { Master-mix } \\
\text { reagents are } \\
\text { dead - i.e. not } \\
\text { thermostable }\end{array}$ & False negative & 9 & $\begin{array}{l}\text { Inactive } \\
\text { reagents, no } \\
\text { amplification }\end{array}$ & 4 & None & 10 & 360 & $\begin{array}{l}\text { Internal } \\
\text { Amplification } \\
\text { Control }\end{array}$ & 9 & 4 & 3 & 108 \\
\hline \multirow{2}{*}{$\begin{array}{l}\text { Sample } \\
\text { collector } \\
\text { device and } \\
\text { TSP/PVI } \\
\text { treatment }\end{array}$} & \multirow{2}{*}{$\begin{array}{l}\text { Contain sample, } \\
\text { Liquefy and } \\
\text { disinfect sputum }\end{array}$} & $\begin{array}{l}\text { Sample not } \\
\text { disinfected }\end{array}$ & $\begin{array}{l}\text { Biohazard risk - } \\
\text { infectious } \\
\text { mycobacteria } \\
\text { may be released }\end{array}$ & 10 & Too little PVI & 3 & None & 10 & 300 & $\begin{array}{l}\text { Metering of sputum } \\
\text { put in an excess of } \\
\text { PVI }\end{array}$ & 10 & 1 & 5 & 50 \\
\hline & & $\begin{array}{l}\text { Sample not } \\
\text { liquefied }\end{array}$ & $\begin{array}{l}\text { Too viscous, } \\
\text { cannot be } \\
\text { processed }\end{array}$ & 5 & Too little TSP & 3 & $\begin{array}{l}\text { Gauge } \\
\text { viscosity of } \\
\text { sample }\end{array}$ & 5 & 75 & $\begin{array}{l}\text { Metering of sputum } \\
\text { put in an excess of } \\
\text { TSP }\end{array}$ & 5 & 1 & 5 & 25 \\
\hline
\end{tabular}




\begin{tabular}{|c|c|c|c|c|c|c|c|c|c|c|c|c|c|c|}
\hline \multicolumn{10}{|c|}{ Risk Estimation and Evaluation } & \multicolumn{5}{|c|}{ Risk Control } \\
\hline ن் & Function & $\begin{array}{l}\text { Failure } \\
\text { Mode }\end{array}$ & Failure Effect & $\mathbf{S}$ & $\begin{array}{l}\text { Cause of } \\
\text { Failure } \\
\text { Mode }\end{array}$ & $\mathbf{O}$ & $\begin{array}{l}\text { Current } \\
\text { Controls }\end{array}$ & D & RPN & $\begin{array}{l}\text { Mitigation } \\
\text { Strategy }\end{array}$ & $\mathbf{S}^{*}$ & $\mathbf{O}^{*}$ & $\mathbf{D}^{*}$ & RPN* \\
\hline \multirow{3}{*}{$\begin{array}{l}\text { Electrolytic } \\
\text { pumps }\end{array}$} & \multirow{3}{*}{$\begin{array}{l}\text { \#1 Pump wash } \\
\text { through } \\
\text { ominvalve } \\
\text { \#2 Fill elution } \\
\text { into valve } \\
\text { \#3 Pump elution } \\
\text { out of valve into } \\
\text { reagent chamber } \\
\text { \#3 Pump } \\
\text { reagents into rxn } \\
\text { chamber } \\
\text { \#4\&5 Pump } \\
\text { amplified } \\
\text { master-mix onto } \\
\text { LF strips }\end{array}$} & \multirow{3}{*}{$\begin{array}{l}\text { Pump loses } \\
\text { pressure, } \\
\text { liquid is not } \\
\text { transferred }\end{array}$} & $\begin{array}{l}\text { Test fails - no } \\
\text { result }\end{array}$ & 7 & $\begin{array}{l}\text { Electrolytes are } \\
\text { not touching, } \\
\text { no voltage } \\
\text { applied, no } \\
\text { flow of } \\
\text { reagents }\end{array}$ & 6 & $\begin{array}{l}\text { Visual } \\
\text { inspection }\end{array}$ & 7 & 294 & $\begin{array}{l}\text { Internal feedback } \\
\text { system } \\
\text { Manually run current } \\
\text { through system } \\
\text { before testing to } \\
\text { ensure electrolytes } \\
\text { are connecting }\end{array}$ & 7 & 3 & 3 & 63 \\
\hline & & & $\begin{array}{l}\text { Test fails - no } \\
\text { result }\end{array}$ & 7 & $\begin{array}{l}\text { Failure at } \\
\text { interconnect } \\
\text { between PP } \\
\text { insert and PC } \\
\text { core }\end{array}$ & 5 & $\begin{array}{l}\text { Visual } \\
\text { inspection }\end{array}$ & 7 & 287 & $\begin{array}{l}\text { Better pressure } \\
\text { applied to connect } \\
\text { insert - locking } \\
\text { mechanism } \\
\text { Screws closer to } \\
\text { insert }\end{array}$ & 7 & 3 & 7 & 147 \\
\hline & & & $\begin{array}{l}\text { False negative } \\
\text { due to inhibitors } \\
\text { carried through }\end{array}$ & 10 & $\begin{array}{l}\text { Inefficient } \\
\text { washing, too } \\
\text { much back } \\
\text { pressure during } \\
\text { that step, due } \\
\text { to beads } \\
\text { settling in } \\
\text { chamber }\end{array}$ & 6 & $\begin{array}{l}\text { Visual } \\
\text { inspection }\end{array}$ & 8 & 480 & $\begin{array}{l}\text { Keep motor running } \\
\text { at } 6 \mathrm{~V} \text { between } \\
\text { sample introductions } \\
\text { and washing so } \\
\text { beads don't settle. } \\
\text { Internal } \\
\text { amplification control }\end{array}$ & 10 & 2 & 1 & 20 \\
\hline
\end{tabular}




\section{APPENDIX F: ROOT CAUSE FAILURE ANALYSIS}

The work presented in this thesis utilized root cause failure analysis to identify causes of unexpected results, categorized as "failures" because something did not perform as expected. The details of all of the experimental set ups and root cause failure analysis are not included in this thesis.

One example where I employed root cause failure analysis is when my initial amplification and detection experiments performed in pouches on the initial cartridge prototype yielded unusually high false negative results, even after very long incubation periods on the heater unit (> 60 minutes). Previous experiments demonstrated EXPAR amplification in pouches on the heater unit, with fluid withdrawn from the pouches to verify amplification via external lateral flow. However, once the amplification pouches and lateral flow detection chambers were integrated into the initial cartridge prototype, the reactions were not amplifying.

The original hypothesis was that the reaction failures were due to improper thermal control of the handheld heater unit or heat conductivity from the heater plate into the reaction fluid was not sufficient. Thermal testing confirmed the heater surface was reaching appropriate assay temperature; therefore, improper heater temperature control was eliminated as a possible source of failure. Cartridges were also run in a temperaturecontrolled water bath, with the same false negative results after $>60$ minutes of incubation, indicating that improper temperature conductivity into the reaction fluid inside the reaction pouches was also not causing the false negative results. All of the cartridge and pouch materials were tested for assay compatibility without any issues, 
signifying that there were no inhibitors leeching from these materials were that could be the source of the false negative results.

During discussion of these false negative results, Hsiang-Wei explained how, in his previous work involving microfluidic PCR chips, his team added BSA into the PCR master-mix reagents to pacify the chip surfaces and prevent enzymes from adhering to the fluid channels. A concentration of $1.6 \mathrm{mg} / \mathrm{mL}$ BSA was chosen based on unrelated DOE studies performed by previous lab member Jose Salazar demonstrating the assay performance was unaffected by the inclusion of BSA. Immediately after incorporating BSA into the cartridge reactions, amplification was restored, with detection of product after 30 minutes of incubation on the heater unit. Therefore, it was concluded that BSA needed to be included into the reaction master-mix in order to pacify the plastic surface area. The inclusion of BSA has become a requirement for all isothermal assays performed on the cartridges, based on this root cause failure analysis. 


\section{APPENDIX G: TRANSFER TO CLEAN MANUFACTURING}

One major concern for developing a nucleic acid amplification diagnostic is the risk of amplicon carry-over contamination. Since the target sequence is exponentially amplified, any breach in containment can leak amplicon product into the environment, leading to false positive results in subsequent tests. Therefore, it is imperative that manufacturing processes are performed under clean environmental conditions without any amplicon contamination in order to ensure proper test results.

While developing the initial amplification and detection prototype subunit, we identified contamination in our manufacturing areas despite our best efforts at amplicon containment. Swab test results indicated that the fluid conduits between the top and bottom cards of the initial cartridge prototype leaked amplicon product. Also, all cartridge components showed amplicon contamination prior to assembly into cartridges, indicating that the manufacturing areas and processes were contaminated.

Consequently, we needed to move cartridge assembly and manufacturing processes into a clean environment. I spear-headed the transition to clean manufacturing, with input from Dr. Angelika Niemz and Barbara Erwin. This entailed first identifying and securing lab space that had not yet been exposed to amplicon product. It was also important that this space have a locking door, so that we could control access to this area.

After cleaning the new lab space, I then secured a laminar flow hood to be used for final cartridge manufacturing and assembly steps. This hood continually blows filtered air and maintains a clean environment inside the workspace. Once the hood was installed, the tools for all cartridge manufacturing processes needed to be replicated inside the hood. This required creating a second heated arbor press set-up for thermal 
bonding, including all pouch dies, and each component was thoroughly cleaned with bleach solution prior to bringing it into the clean lab.

Furthermore, all cartridge materials needed to be ordered to replace the materials we had been using in the contaminated lab areas. For the components that were directly assembled into the cartridges, such as plastic films and silicone foam, these items were carefully handled upon receipt and were directly moved into the clean room for storage to eliminate any possible amplicon exposure from contaminated lab areas. For components that required assembly and manipulation or testing prior to assembly into the cartridges, such as valves and septa, I developed a decontamination process. In previous studies, we demonstrated that dry-autoclaving effectively eliminates contamination. Therefore, all of these subassembly components were first manufactured and tested, then autoclaved and then brought directly into the clean lab for assembly into the cartridges.

After all of the components were replaced or decontaminated and all of the process tools were brought into the clean environment, I refined the manufacturing protocol for this environment. Once the process was optimized, I was able to manufacture clean initial cartridge prototypes and I demonstrated that these cartridges were not contaminated with amplicon product. In total, the transition to clean manufacturing required approximately one year until the first clean cartridges were fully-assembled and tested.

We have additionally transitioned the manufacturing of the refined cartridge prototype into this clean environment. As mentioned in Chapter 4, we are still optimizing this process. Our next step will be to design the manufacturing processes for the fullyintegrated cartridges in this clean lab. 


\section{REFERENCES}

1. Ince, J.; McNally, A. Development of rapid, automated diagnostics for infectious disease: advances and challenges. Expert Review of Medical Devices 2009, 6 (6), 641-651.

2. Kaltenboeck, B.; Wang, C. M. Advances in real-time PCR: Application to clinical laboratory diagnostics. Adv. Clin. Chem. 2005, 40, 219-259.

3. de Tejada, B. M.; Stan, C. M.; Boulvain, M.; Renzi, G.; Francois, P.; Irion, O.; Schrenzel, J. Development of a Rapid PCR Assay for Screening of Maternal Colonization by Group B Streptococcus and Neonatal Invasive Escherichia coli during Labor. Gynecol. Obstet. Invest. 2010, 70 (4), 250-255.

4. Wolk, D. M.; Marx, J. L.; Dominguez, L.; Driscoll, D.; Schifman, R. B. Comparison of MRSASelect Agar, CHROMagar Methicillin-Resistant Staphylococcus aureus (MRSA) Medium, and Xpert MRSA PCR for Detection of MRSA in Nares:

Diagnostic Accuracy for Surveillance Samples with Various Bacterial Densities. $J$. Clin. Microbiol. 2009, 47 (12), 3933-3936.

5. Brenwald, N. P.; Baker, N.; Oppenheim, B. Feasibility study of a real-time PCR test for methicillin-resistant Staphylococcus aureus in a point of care setting. J. Hosp. Infect. 2010, 74 (3), 245-249.

6. Yager, P.; Domingo, G. J.; Gerdes, J. Point-of-care diagnostics for global health. Annual Review of Biomedical Engineering 2008, 10, 107-144.

7. Puren, A.; Gerlach, J. L.; Weigl, B. H.; Kelso, D. M.; Domingo, G. J. Laboratory Operations, Specimen Processing, and Handling for Viral Load Testing and Surveillance. J. Infect. Dis. 2010, 201, S27-S36.

8. Stevens, W. S.; Marshall, T. M. Challenges in Implementing HIV Load Testing in South Africa. J. Infect. Dis. 2010, 201, S78-S84.

9. Maartens, G.; Wilkinson, R. J. Tuberculosis. Lancet 2007, 370 (9604), 2030-2043.

10. World Health Organization Global Tuberculosis Report; 12.

11. McNerney, R.; Daley, P. Towards a point-of-care test for active tuberculosis: obstacles and opportunities. Nature Reviews Microbiology 2011, 9 (3), 204-213.

12. Perkins, M. D.; Cunningham, J. Facing the crisis: Improving the diagnosis of tuberculosis in the HIV era. J. Infect. Dis. 2007, 196, S15-S27.

13. Balasingham, S. V.; Davidsen, T.; Szpinda, I.; Frye, S. A.; Tonjuin, T. Molecular Diagnostics in Tuberculosis Basis and Implications for Therapy. Molecular Diagnosis \& Therapy 2009, 13 (3), 137-151.

14. Boehme, C. C.; Nabeta, P.; Hillemann, D.; Nicol, M. P.; Shenai, S.; Krapp, F.; Allen, J.; Tahirli, R.; Blakemore, R.; Rustomjee, R.; Milovic, A.; Jones, M.; O'Brien, S. M.; Persing, D. H.; Ruesch-Gerdes, S.; Gotuzzo, E.; Rodrigues, C.; Alland, D.; Perkins, M. D. Rapid Molecular Detection of Tuberculosis and Rifampin Resistance. N. Engl. J. Med. 2010, 363 (11), 1005-1015.

15. Blakemore, R.; Story, E.; Helb, D.; Kop, J.; Banada, P.; Owens, M. R.; Chakravorty, S.; Jones, M.; Alland, D. Evaluation of the Analytical Performance of the Xpert MTB/RIF Assay. J. Clin. Microbiol. 2010, 48 (7), 2495-2501.

16. Raja, S.; Ching, J.; Xi, L. Q.; Hughes, S. J.; Chang, R.; Wong, W.; McMillan, W.; Gooding, W. E.; McCarty, K. S.; Chestney, M.; Luketich, J. D.; Godfrey, T. E. 
Technology for automated, rapid, and quantitative PCR or reverse transcription-PCR clinical testing. Clin. Chem. 2005, 51 (5), 882-890.

17. Helb, D.; Jones, M.; Story, E.; Boehme, C.; Wallace, E.; Ho, K.; Kop, J.; Owens, M. R.; Rodgers, R.; Banada, P.; Safi, H.; Blakemore, R.; Lan, N. T. N.; Jones-Lopez, E. C.; Levi, M.; Burday, M.; Ayakaka, I.; Mugerwa, R. D.; McMillan, B.; Winn-Deen, E.; Christel, L.; Dailey, P.; Perkins, M. D.; Persing, D. H.; Alland, D. Rapid Detection of Mycobacterium tuberculosis and Rifampin Resistance by Use of OnDemand, Near-Patient Technology. J. Clin. Microbiol. 2010, 48 (1), 229-237.

18. Mwaba, P.; McNerney, R.; Grobusch, M. P.; O'Grady, J.; Bates, M.; Kapata, N.; Maeurer, M.; Zumla, A. Achieving STOP TB Partnership goals: perspectives on development of new diagnostics, drugs and vaccines for tuberculosis. Tropical Medicine \& International Health 2011, 16 (7), 819-827.

19. Niemz, A.; Boyle, D. S. Nucleic acid testing for tuberculosis at the point-of-care in high burden countries. Expert Rev. Mol. Diagn. 2012, 687-701.

20. Niemz, A.; Ferguson, T. M.; Boyle, D. S. Point-of-care nucleic acid testing for infectious diseases. Trends Biotechnol. 2011, 29 (5), 240-250.

21. Poritz, M. A.; Blaschke, A. J.; Byington, C. L.; Meyers, L.; Nilsson, K.; Jones, D. E.; Thatcher, S. A.; Robbins, T.; Lingenfelter, B.; Amiott, E.; Herbener, A.; Daly, J.; Dobrowolski, S. F.; David, H. F. T.; Ririe, K. M. FilmArray, an Automated Nested Multiplex PCR System for Multi-Pathogen Detection: Development and Application to Respiratory Tract Infection. Plos One 2011, 6 (10).

22. Tanriverdi, S.; Chen, L. J.; Chen, S. Q. A Rapid and Automated Sample-to-Result HIV Load Test for Near-Patient Application. J. Infect. Dis. 2010, 201, S52-S58.

23. Asiello, P. J.; Baeumner, A. J. Miniaturized isothermal nucleic acid amplification, a review. Lab on A Chip 2011, 11 (8), 1420-1430.

24. Mahalanabis, M.; Do, J.; ALMuayad, H.; Zhang, J. Y.; Klapperich, C. M. An integrated disposable device for DNA extraction and helicase dependent amplification. Biomed. Microdevices. 2010, 12 (2), 353-359.

25. Wang, C. H.; Lien, K. Y.; Wu, J. J.; Lee, G. B. A magnetic bead-based assay for the rapid detection of methicillin-resistant Staphylococcus aureus by using a microfluidic system with integrated loop-mediated isothermal amplification. Lab Chip. 2011, 11 (8), 1521-1531.

26. Wu, Q.; Jin, W.; Zhou, C.; Han, S.; Yang, W.; Zhu, Q.; Jin, Q.; Mu, Y. Integrated glass microdevice for nucleic acid purification, loop-mediated isothermal amplification, and online detection. Anal. Chem. 2011, 83 (9), 3336-3342.

27. Boyanton, B. L.; Sural, P.; Loomis, C. R.; Pesta, C.; Gonzalez-Krellwitz, L.; Robinson-Dunn, B.; Riska, P. Loop-Mediated Isothermal Amplification Compared to Real-Time PCR and Enzyme Immunoassay for Toxigenic Clostridium difficile Detection. J. Clin. Microbiol. 2012, 50 (3), 640-645.

28. Hicke, B.; Pasko, C.; Groves, B.; Ager, E.; Corpuz, M.; Frech, G.; Munns, D.; Smith, W.; Warcup, A.; Denys, G.; Ledeboer, N. A.; Lindsey, W.; Owen, C.; Rea, L.; Jenison, R. Automated Detection of Toxigenic Clostridium difficile in Clinical Samples: Isothermal tcdB Amplification Coupled to Array-Based Detection. J. Clin. Microbiol. 2012, 50 (8), 2681-2687.

29. Kim, H. J.; Tong, Y. H.; Tang, W.; Quimson, L.; Cope, V. A.; Pan, X. J.; Motre, A.; Kong, R.; Hong, J. A.; Kohn, D.; Miller, N. S.; Poulter, M. D.; Kong, H. M.; Tang, Y. 
W.; Yen-Lieberman, B. A rapid and simple isothermal nucleic acid amplification test for detection of herpes simplex virus types 1 and 2. J. Clin. Virol. 2011, 50 (1), 26-30.

30. Burns, M. A.; Johnson, B. N.; Brahmasandra, S. N.; Handique, K.; Webster, J. R.; Krishnan, M.; Sammarco, T. S.; Man, P. M.; Jones, D.; Heldsinger, D.; Mastrangelo, C. H.; Burke, D. T. An integrated nanoliter DNA analysis device. Science 1998, 282 (5388), 484-487.

31. Easley, C. J.; Karlinsey, J. M.; Bienvenue, J. M.; Legendre, L. A.; Roper, M. G.; Feldman, S. H.; Hughes, M. A.; Hewlett, E. L.; Merkel, T. J.; Ferrance, J. P.; Landers, J. P. A fully integrated microfluidic genetic analysis system with sample-in-answerout capability. Proc. Natl. Acad. Sci. U. S. A. 2006, 103 (51), 19272-19277.

32. Yobas, L.; Ji, H. M.; Hui, W. C.; Chen, Y.; Lim, T. M.; Heng, C. K.; Kwong, D. L. Nucleic acid extraction, amplification, and detection on Si-based microfluidic platforms. Ieee Journal of Solid-State Circuits 2007, 42 (8), 1803-1813.

33. Chen, D. F.; Mauk, M.; Qiu, X. B.; Liu, C. C.; Kim, J. T.; Ramprasad, S.; Ongagna, S.; Abrams, W. R.; Malamud, D.; Corstjens, P. L. A. M.; Bau, H. H. An integrated, self-contained microfluidic cassette for isolation, amplification, and detection of nucleic acids. Biomedical Microdevices 2010, 12 (4), 705-719.

34. Burns, M. A.; Johnson, B. N.; Brahmasandra, S. N.; Handique, K.; Webster, J. R.; Krishnan, M.; Sammarco, T. S.; Man, P. M.; Jones, D.; Heldsinger, D.; Mastrangelo, C. H.; Burke, D. T. An integrated nanoliter DNA analysis device. Science 1998, 282 (5388), 484-487.

35. Marcus, J. S.; Anderson, W. F.; Quake, S. R. Parallel picoliter RT-PCR assays using microfluidics. Anal. Chem. 2006, 78 (3), 956-958.

36. Scarparo, C.; Piccoli, P.; Rigon, A.; Ruggiero, G.; Scagnelli, M.; Piersimoni, C. Comparison of enhanced Mycobacterium tuberculosis amplified direct test with COBAS AMPLICOR Mycobacterium tuberculosis assay for direct detection of Mycobacterium tuberculosis complex in respiratory and extrapulmonary specimens. $J$. Clin. Microbiol. 2000, 38 (4), 1559-1562.

37. Mori, Y.; Notomi, T. Loop-mediated isothermal amplification (LAMP): a rapid, accurate, and cost-effective diagnostic method for infectious diseases. Journal of Infection and Chemotherapy 2009, 15 (2), 62-69.

38. Iwamoto, T.; Sonobe, T.; Hayashi, K. Loop-mediated isothermal amplification for direct detection of Mycobacterium tuberculosis complex, M-avium, and Mintracellulare in sputum samples. J. Clin. Microbiol. 2003, 41 (6), 2616-2622.

39. Boehme, C. C.; Nabeta, P.; Henostroza, G.; Raqib, R.; Rahim, Z.; Gerhardt, M.; Sanga, E.; Hoelscher, M.; Notomi, T.; Hase, T.; Perkins, M. D. Operational feasibility of using loop-mediated isothermal amplification for diagnosis of pulmonary tuberculosis in microscopy centers of developing countries. J. Clin. Microbiol. 2007, 45 (6), 1936-1940.

40. Mitarai, S.; Okumura, M.; Toyota, E.; Yoshiyama, T.; Aono, A.; Sejimo, A.; Azuma, Y.; Sugahara, K.; Nagasawa, T.; Nagayama, N.; Yamane, A.; Yano, R.; Kokuto, H.; Morimoto, K.; Ueyama, M.; Kubota, M.; Yi, R.; Ogata, H.; Kudoh, S.; Mori, T. Evaluation of a simple loop-mediated isothermal amplification test kit for the diagnosis of tuberculosis. Int. J Tuberc. Lung Dis. 2011, 15 (9), 1211-1217. 
41. Dineva, M. A.; Candotti, D.; Fletcher-Brown, F.; Allain, J. P.; Lee, H. Simultaneous visual detection of multiple viral amplicons by dipstick assay. J. Clin. Microbiol. 2005, 43 (8), 4015-4021.

42. Fang, R. D.; Li, X.; Hu, L.; You, Q. M.; Li, J.; Wu, J.; Xu, P.; Zhong, H. Y.; Luo, Y.; Mei, J.; Gao, Q. Cross-Priming Amplification for Rapid Detection of Mycobacterium tuberculosis in Sputum Specimens. J. Clin. Microbiol. 2009, 47 (3), 845-847.

43. Goldmeyer, J.; Li, H.; McCormac, M.; Cook, S.; Stratton, C.; Lemieux, B.; Kong, H.; Tang, W.; Tang, Y. W. Identification of Staphylococcus aureus and determination of methicillin resistance directly from positive blood cultures by isothermal amplification and a disposable detection device. J. Clin. Microbiol. 2008, 46 (4), 1534-1536.

44. Mugasa, C. M.; Laurent, T.; Schoone, G. J.; Kager, P. A.; Lubega, G. W.; Schallig, H. D. F. H. Nucleic Acid Sequence-Based Amplification with Oligochromatography for Detection of Trypanosoma brucei in Clinical Samples. J. Clin. Microbiol. 2009, 47 (3), 630-635.

45. Piepenburg, O.; Williams, C. H.; Stemple, D. L.; Armes, N. A. DNA detection using recombination proteins. Plos Biology 2006, 4 (7), 1115-1121.

46. Puthawibool, T.; Senapin, S.; Kiatpathomchai, W.; Flegel, T. W. Detection of shrimp infectious myonecrosis virus by reverse transcription loop-mediated isothermal amplification combined with a lateral flow dipstick. J. Virol. Methods 2009, 156 (1-2), 27-31.

47. Nimitphak, T.; Meemetta, W.; Arunrut, N.; Senapin, S.; Kiatpathomchai, W. Rapid and sensitive detection of Penaeus monodon nucleopolyhedrovirus (PemoNPV) by loop-mediated isothermal amplification combined with a lateral-flow dipstick. Mol. Cell. Probes 2010, 24 (1), 1-5.

48. Prompamorn, P.; Sithigorngul, P.; Rukpratanporn, S.; Longyant, S.; Sridulyakul, P.; Chaivisuthangkura, P. The development of loop-mediated isothermal amplification combined with lateral flow dipstick for detection of Vibrio parahaemolyticus. Lett. Appl. Microbiol. 2011, 52 (4), 344-351.

49. Jaroenrama, W.; Kiatpathomchai, W.; Flegel, T. W. Rapid and sensitive detection of white spot syndrome virus by loop-mediated isothermal amplification combined with a lateral flow dipstick. Mol. Cell. Probes 2009, 23 (2), 65-70.

50. Lee, H. H.; Dineva, M. A.; Chua, Y. L.; Ritchie, A. V.; Ushiro-Lumb, I.; Wisniewski, C. A. Simple Amplification-Based Assay: A Nucleic Acid Based Point-of-Care Platform for HIV-1 Testing. J. Infect. Dis. 2010, 201, S65-S72.

51. Rand, K. H.; Rampersaud, H.; Houck, H. J. Comparison of Two Multiplex Methods for Detection of Respiratory Viruses: FilmArray RP and xTAG RVP. J. Clin. Microbiol. 2011, 49 (7), 2449-2453.

52. Zhou, P.; Young, L.; Chen, Z. Y. Weak solvent based chip lamination and characterization of on-chip valve and pump. Biomedical Microdevices 2010, 12 (5), 821-832.

53. Böhm, S.; Olthuis, W.; Bergveld, P. An Integrated Micromachined Electrochemical Pump and Dosing System. Biomedical Microdevices 1999, 1 (2), 121-130.

54. Liu, R. H.; Yang, J. N.; Lenigk, R.; Bonanno, J.; Grodzinski, P. Self-contained, fully integrated biochip for sample preparation, polymerase chain reaction amplification, and DNA microarray detection. Anal. Chem. 2004, 76 (7), 1824-1831. 
55. Liu, R. H.; Nguyen, T.; Schwarzkopf, K.; Fuji, H. S.; Petrova, A.; Siuda, T.; Peyvan, K.; Bizak, M.; Danley, D.; McShea, A. Fully integrated miniature device for automated gene expression DNA microarray processing. Anal. Chem. 2006, 78 (6), 1980-1986.

56. Liu, R. H.; Lodes, M. J.; Nguyen, T.; Siuda, T.; Slota, M.; Fuji, H. S.; McShea, A. Validation of a fully integrated microfluidic array device for influenza A subtype identification and sequencing. Anal. Chem. 2006, 78 (12), 4184-4193.

57. Vandeventer, P. E.; Weigel, K. M.; Salazar, J.; Erwin, B.; Irvine, B.; Doebler, R.; Nadim, A.; Cangelosi, G. A.; Niemz, A. Mechanical Disruption of Lysis-Resistant Bacterial Cells by Use of a Miniature, Low-Power, Disposable Device. J. Clin. Microbiol. 2011, 49 (7), 2533-2539.

58. Van Ness, J.; Van Ness, L. K.; Galas, D. J. Isothermal reactions for the amplification of oligonucleotides. Proc. Natl. Acad. Sci. U. S. A. 2003, 100 (8), 4504-4509.

59. Tan, E.; Wong, J.; Nguyen, D.; Zhang, Y.; Erwin, B.; Van Ness, L. K.; Baker, S. M.; Galas, D. J.; Niemz, A. Isothermal DNA amplification coupled with DNA nanosphere-based colorimetric detection. Anal. Chem. 2005, 77 (24), 7984-7992.

60. Spiro, A.; Lowe, M.; Brown, D. A bead-based method for multiplexed identification and quantitation of DNA sequences using flow cytometry. Appl. Environ. Microbiol. 2000, 66 (10), 4258-4265.

61. Tan, E.; Erwin, B.; Dames, S.; Voelkerding, K.; Niemz, A. Isothermal DNA amplification with gold nanosphere-based visual colorimetric readout for herpes simplex 2 virus detection. Clin. Chem. 2007, 53 (11), 2017-2020.

62. Lu, H. W.; Roskos, K.; Hickerson, A. I.; Carey, T.; Niemz, A. System for Portable Nucleic Acid Testing in Low Resource Settings. SPIE: Bellingham, Washington, 2013.

63. Hickerson, A. I.; Lu, H. W.; Roskos, K.; Carey, T.; Niemz, A. Disposable Miniature Check Valve Design Suitable For Scalable Manufacturing. 2011; p under review.

64. Beebe, D. J.; Mensing, G. A.; Walker, G. M. Physics and applications of microfluidics in biology. Annual Review of Biomedical Engineering 2002, 4, 261-286.

65. Oh, K. W.; Rong, R.; Ahn, C. H. Miniaturization of pinch-type valves and pumps for practical micro total analysis system integration. Journal of Micromechanics and Microengineering 2005, 15 (12), 2449-2455.

66. Oh, K. W.; Ahn, C. H. A review of microvalves. Journal of Micromechanics and Microengineering 2006, 16 (5), R13-R39.

67. Yang, X.; Grosjean, C.; Tai, Y. C. Design, fabrication, and testing of micromachined silicone rubber membrane valves. Journal of Microelectromechanical Systems 1999, $8(4), 393-402$.

68. Yuen, P. K.; Kricka, L. J.; Wilding, P. Semi-disposable microvalves for use with microfabricated devices or microchips. Journal of Micromechanics and Microengineering 2000, 10 (3), 401-409.

69. Chin, C. D.; Linder, V.; Sia, S. K. Commercialization of microfluidic point-of-care diagnostic devices. Lab on A Chip 2012, 12 (12), 2118-2134.

70. Gervais, L.; de Rooij, N.; Delamarche, E. Microfluidic Chips for Point-of-Care Immunodiagnostics. Advanced Materials 2011, 23 (24), H151-H176. 
71. Hasselbrink, E. F.; Shepodd, T. J.; Rehm, J. E. High-pressure microfluidic control in lab-on-a-chip devices using mobile polymer monoliths. Anal. Chem. 2002, 74 (19), 4913-4918.

72. Koch, M.; Evans, A. G. R.; Brunnschweiler, A. Characterization of micromachined cantilever valves. Journal of Micromechanics and Microengineering 1997, 7 (3), 221-223.

73. Nguyen, N. T.; Truong, T. Q.; Wong, K. K.; Ho, S. S.; Low, C. L. N. Micro check valves for integration into polymeric microfluidic devices. Journal of Micromechanics and Microengineering 2004, 14 (1), 69-75.

74. Oosterbroek, R. E.; Berenschot, J. W.; Schlautmann, S.; Krijnen, G. J. M.; Lammerink, T. S. J.; Elwenspoek, M. C.; van den Berg, A. Designing, simulation and realization of in-plane operating micro valves, using new etching techniques. Journal of Micromechanics and Microengineering 1999, 9 (2), 194-198.

75. Snakenborg, D.; Klank, H.; Kutter, J. P. Polymer microvalve with pre-stressed membranes for tunable flow-pressure characteristics. Microfluidics and Nanofluidics 2011, 10 (2), 381-388.

76. Wang, J.; Chen, Z. Y.; Mauk, M.; Hong, K. S.; Li, M. Y.; Yang, S.; Bau, H. H. Selfactuated, thermo-responsive hydrogel valves for lab on a chip. Biomedical Microdevices 2005, 7 (4), 313-322.

77. Brandes, R. V. Annular One-Way Valve. US 7,243,682 B2, Jul 17, 2007.

78. Epstein, A. B. One-Way Valve. US 5,660,205, Aug 26, 1997.

79. Floh, R.; Spindler, R. In-Line Check Valve. US 6,848,471 B2, Feb 1, 2005.

80. Roskos, K.; Hickerson, A. I.; Lu, H. W.; Ferguson, T. M.; Shinde, D. N.; Klaue, Y.; Niemz, A. Simple System for Isothermal DNA Amplification Coupled to Lateral Flow Detection. 2013; p under review.

81. Qiu, X. B.; Chen, D. F.; Liu, C. C.; Mauk, M. G.; Kientz, T.; Bau, H. H. A portable, integrated analyzer for microfluidic - based molecular analysis. Biomedical Microdevices 2011, 13 (5), 809-817.

82. Lutz, S.; Weber, P.; Focke, M.; Faltin, B.; Hoffmann, J.; Muller, C.; Mark, D.; Roth, G.; Munday, P.; Armes, N.; Piepenburg, O.; Zengerle, R.; von Stetten, F.

Microfluidic lab-on-a-foil for nucleic acid analysis based on isothermal recombinase polymerase amplification (RPA). Lab on A Chip 2010, 10 (7), 887-893.

83. Deng, X.; Pack, T. D. Stable Reagents and Kits Useful in Loop-Mediated Isothermal Amplification (LAMP). US 2008/0182313 A1, Jul 31, 2008.

84. Qiu, X. B.; Mauk, M. G.; Chen, D. F.; Liu, C. C.; Bau, H. H. A large volume, portable, real-time PCR reactor. Lab on A Chip 2010, 10 (22), 3170-3177.

85. Buitink, J.; van den Dries, I. J.; Hoekstra, F. A.; Alberda, M.; Hemminga, M. A. High critical temperature above T-g may contribute to the stability of biological systems. Biophys. J. 2000, 79 (2), 1119-1128.

86. Weigl, B. H.; Gerdes, J.; Tarr, P.; Yager, P.; Dillman, L.; Peck, R.; Ramachandran, S.; Lemba, M.; Nabavi, M.; Battrell, F.; Hoekstra, D.; Klein, E. J.; Denno, D. M. Fully integrated multiplexed lab-on-a-card assay for enteric pathogens. 2006; p 611202.

87. Garcia, E.; Kirkham, J. R.; Hatch, A. V.; Hawkins, K. R.; Yager, P. Controlled microfluidic reconstitution of functional protein from an anhydrous storage depot. Lab on A Chip 2004, 4 (1), 78-82. 
88. Kiviniemi, M.; Ilonen, J.; Lovgren, T. A homogeneous HLA-B*27 genotyping assay using dried reagent mixtures. Dis. Markers 2009, 27 (2), 85-91.

89. Kim, J.; Byun, D.; Mauk, M. G.; Bau, H. H. A disposable, self-contained PCR chip. Lab on A Chip 2009, 9 (4), 606-612.

90. Ponaka, R.; Farchaus, J. W.; Pierce, M. D. Preparation of Glassified Biological Reagents. US 2009/0325263 A1, Dec 31, 2009.

91. Treml, S. B.; Dall, C. J.; Draveling, C. A.; Jolly, J. F.; Ramanujam, R. P. Biological Reagent Spheres. US 5,593,824, Jan 14, 1997.

92. Franks, F.; Hatley, R. H. M. Storage of Materials. US 5,098,893, Mar 24, 1992.

93. Walker, D. W.; DiFrancesco, R. A.; Heaster, J. A.; Jolly, J. F.; Lively, C. R.; Treml, S. B. Room Temperature Stable Reagent Semi-Spheres. US 5,565,318, Oct 15, 1996.

94. Bensimon, D.; Kadanoff, L. P.; Liang, S. D.; Shraiman, B. I.; Tang, C. Viscous Flows in 2 Dimensions. Reviews of Modern Physics 1986, 58 (4), 977-999.

95. Casademunt, J.; Magdaleno, F. X. Dynamics and selection of fingering patterns. Recent developments in the Saffman-Taylor problem. Physics Reports-Review Section of Physics Letters 2000, 337 (1-2), 1-35.

96. Hele-Shaw, H. S. The flow of water. Nature 1898, 58 (1489), 34-36.

97. Mclean, J. W.; Saffman, P. G. The Effect of Surface-Tension on the Shape of Fingers in A Hele Shaw Cell. Journal of Fluid Mechanics 1981, 102 (JAN), 455-469.

98. Niemz, A.; Hickerson, A. I.; Roskos, K.; Lu, H. W. System and Method for Efficient Nucleic Acid Testing. Apr 10, 2013. US Patent Application 13860453.

99. Hickerson, A. I. Check Valve. Apr 9, 2012. US Patent Application 61/621,989. 